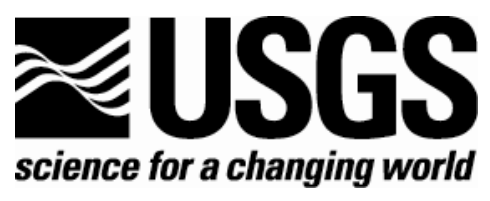

\title{
Mineral production and mining trends for selected non-fuel commodities in Idaho and Montana, 1905-2001
}

By Jeremy C. Larsen, Keith R. Long, Kenneth C. Assmus, and Michael L. Zientek

\section{Open-File Report 2004-1414}

2004

U.S. Department of the Interior

U.S. Geological Survey

Any use of trade, firm, or product names is for descriptive purposes only and does not imply endorsement by the U.S. Government 


\section{U.S. Department of the Interior \\ Gale A. Norton, Secretary}

\section{U.S. Geological Survey \\ Charles G. Groat, Director}

U.S. Geological Survey, Reston, Virginia 2004

Revised and reprinted: 2004

This report and any updates to it are available on the

World Wide Web: http://pubs.usgs.gov/of/2004/1414/

For more information on the USGS - the Federal source for science about the Earth, its natural and living resources, natural hazards, and the environment:

World Wide Web: http://www.usgs.gov

Telephone: 1-888-ASK-USGS

Although this report is in the public domain, permission must be secured from the individual copyright owners to reproduce any copyrighted material contained within this report. 


\section{Contents}

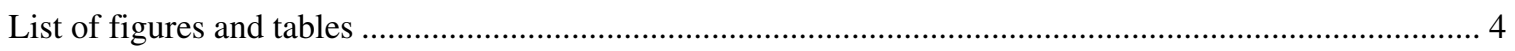

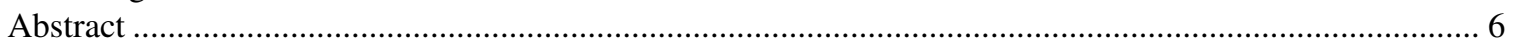

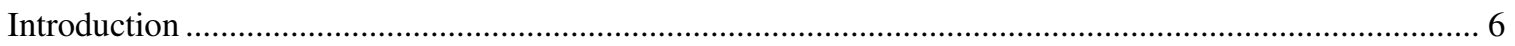

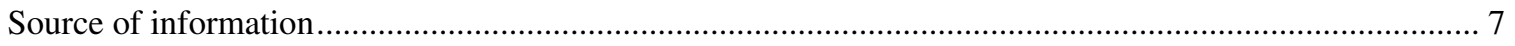

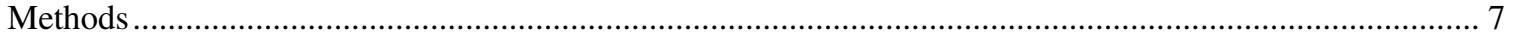

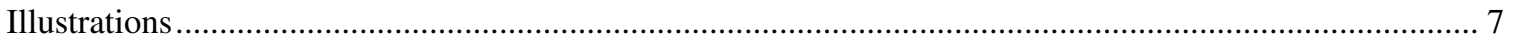

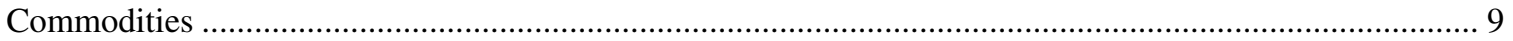

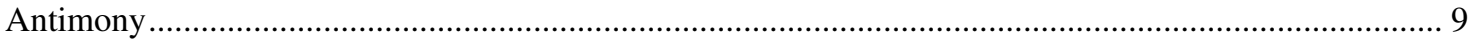

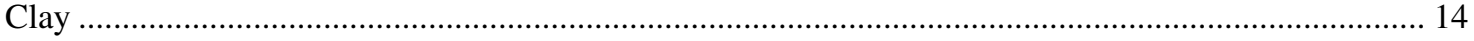

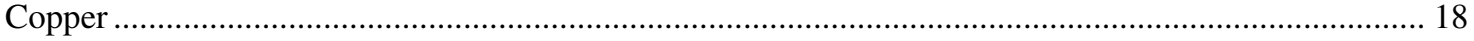

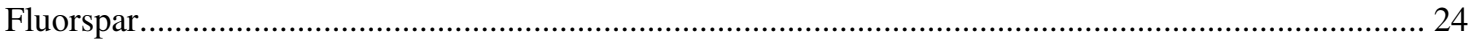

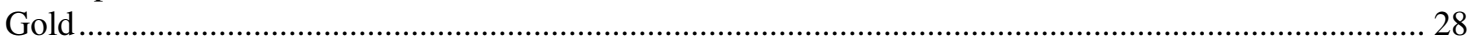

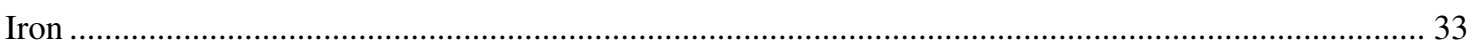

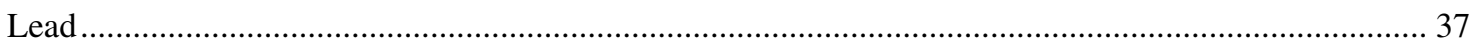

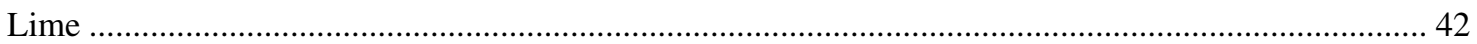

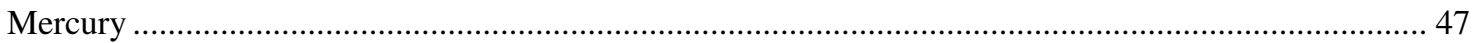

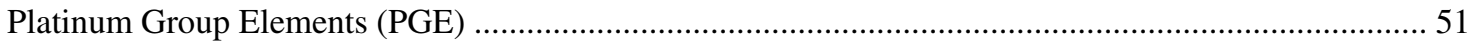

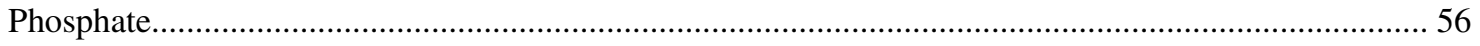

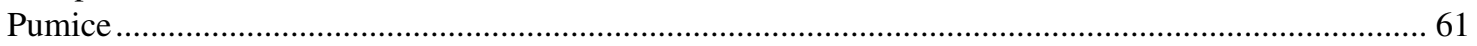

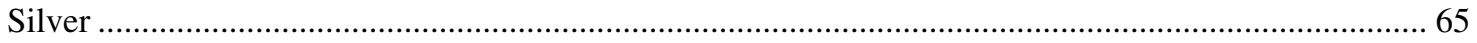

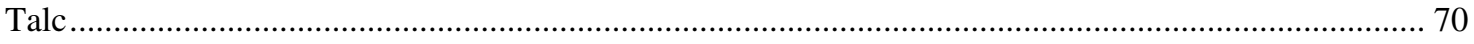

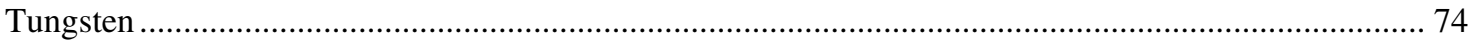

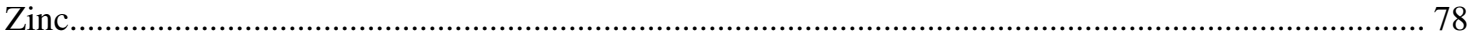

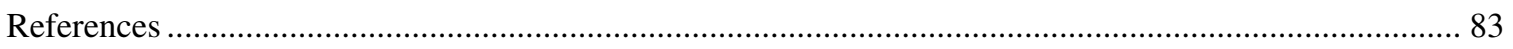

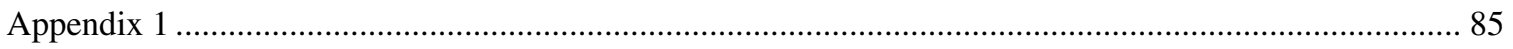

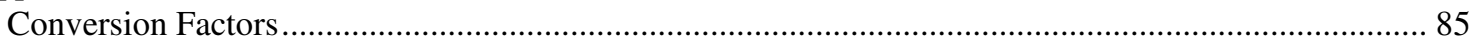




\section{List of figures and tables}

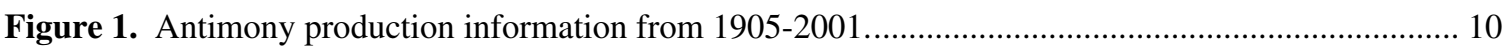

Figure 2. Clay production information from 1905-2001............................................................... 14

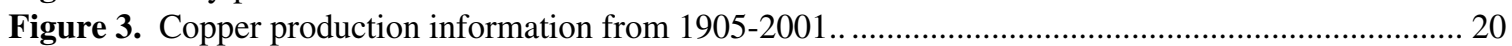

Figure 4. Nineteenth century copper production and associated events (from Goonan, 2002) .................. 21

Figure 5. Fluorspar production information from 1905-2001........................................................... 25

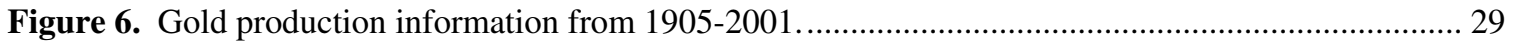

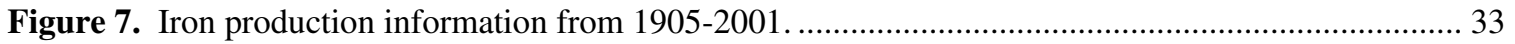

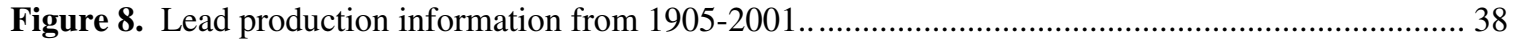

Figure 9. Lime production information from 1905-2001..................................................................... 43

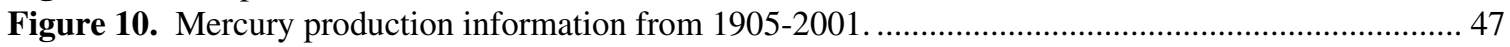

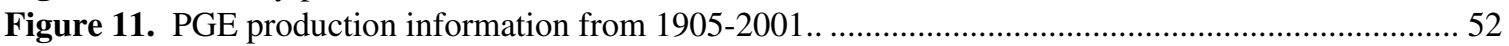

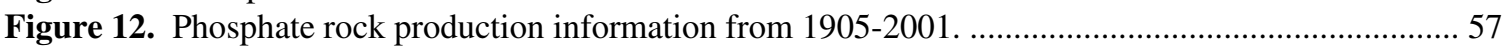

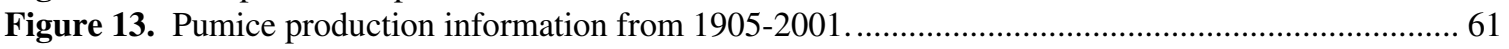

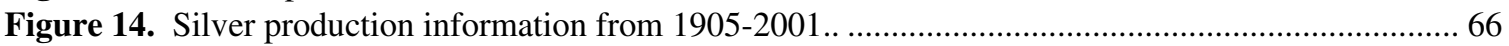

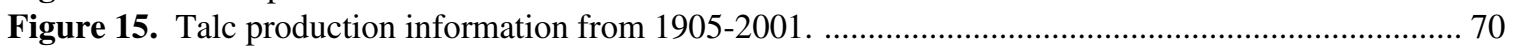

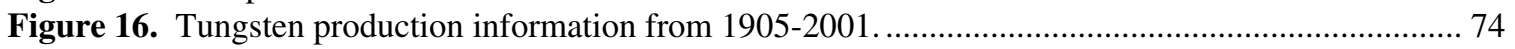

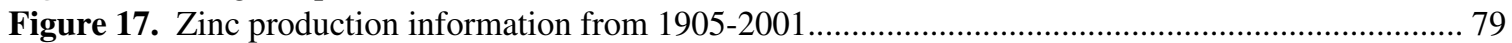

Table 1. Selected events affecting regional antimony production ....................................................... 9

Table 2. Selected events affecting U.S. antimony production .............................................................. 9

Table 3. Antimony production from Idaho and Montana, the U.S., and the world, $1905-2001$.............. 11

Table 4. Clay production from Idaho, Montana, and the U.S., 1905-2001 ............................................. 15

Table 5. Selected events affecting regional copper production............................................................... 18

Table 6. Selected events affecting U.S. copper production ................................................................... 18

Table 7. Copper production, in metric tons, from the Continental mine, 1995 to 1999 (ASARCO, 1999) 19

Table 8. Copper production from Idaho and Montana, the U.S., and the world, 1905-2000 …................ 21

Table 9. Selected events affecting regional fluorspar production ............................................................. 24

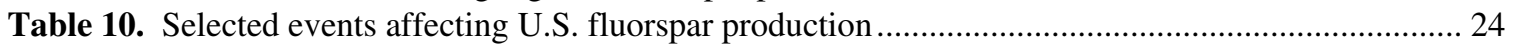

Table 11. Fluorspar production from Idaho and Montana, the U.S., and the world, 1905-2000............... 25

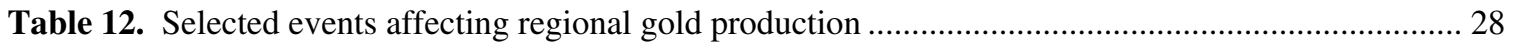

Table 13. Selected events affecting U.S. gold production ……............................................................ 28

Table 14. Gold production from Idaho and Montana, the U.S., and the world, 1905-2000 ……................ 30

Table 15. Iron production from Montana, the U.S., and the world, 1905-2000 …................................... 33

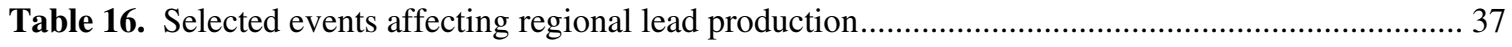

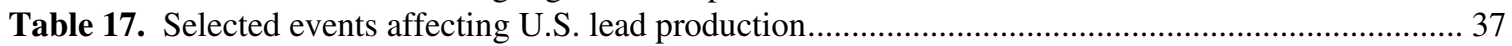

Table 18. Lead production from Idaho and Montana, the U.S., and the world, 1905-2001 ...................... 39

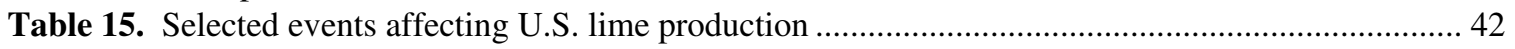

Table 20. Lime production from Idaho and Montana, the U.S., and the world, 1905-2001...................... 44

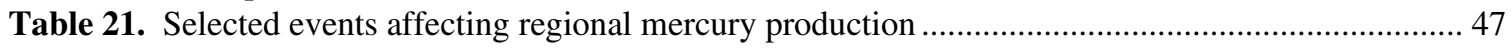

Table 22. Selected events affecting U.S. mercury production .............................................................. 47

Table 23. Mercury production from Idaho, the U.S., and the world, 1905-2001 ...................................... 48

Table 24. Selected events affecting regional PGE production................................................................ 51

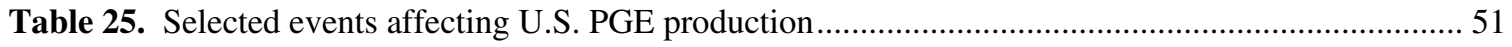

Table 26. PGE production from Montana, the U.S., and the world, 1905-2001 ...................................... 53

Table 27. Selected events affecting regional phosphate rock production ................................................. 56 
Table 28. Selected events affecting U.S. phosphate rock production .................................................56

Table 29. Phosphate rock production from Idaho and Montana, the U.S., and the world, 1905-2001...... 58

Table 30. Pumice production from Idaho, the U.S., and the world, 1905-2001 ................................62

Table 31. Selected events affecting regional silver production...........................................................6 65

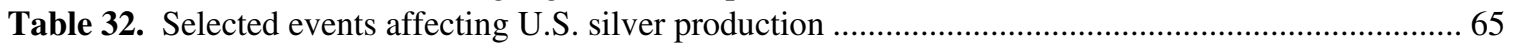

Table 33. Silver production from Idaho and Montana, the U.S., and the world, 1905-2001 ....................67

Table 34. Talc production from Montana, the U.S., and the world, 1905-2000.................................. 71

Table 35. Selected events affecting U.S. tungsten production .................................................... 74

Table 36. Tungsten (60-percent concentrate) production from Idaho and Montana, the U.S. and the world,

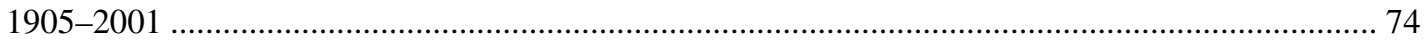

Table 37. Selected events affecting regional zinc production......................................................... 78

Table 38. Selected events affecting U.S. zinc production..................................................... 78

Table 39. Zinc production from Idaho and Montana, the U.S., and the world, 1905-2001.................... 80 


\section{Abstract}

Idaho and Montana state mining statistics were obtained from historical mineral production records and compiled into a continuous record from 1905 through 2001. To facilitate comparisons, the mineral production data were normalized by converting the units of measure to metric tons for all included commodities. These standardized statistical data include production rates for principal non-fuel mineral commodities from both Idaho and Montana, as well as the production rates of similar commodities for the U.S. and the world for contrast. Data are presented here in both tabular and bar chart format. Moreover, the tables of standardized mineral production data are also provided in digital format as, commodity_production.xls.

Some significant historical events pertaining to the mining industry are described as well. When taken into account with the historical production data, this combined information may to help explain both specific fluctuations and general tendencies in the overall trends in the rates of mineral resource production over time.

\section{Introduction}

We have compiled and standardized a continuous record of historical mineral production data for both Idaho and Montana covering a time period from 1905 through 2001. This data includes the primary mineral products and the quantity of major non-fuel minerals and metals produced from mines in both Idaho and Montana (together referred to herein as the "region"), as well as rates for U.S. and world production. From this data, a series of tables and illustrations are presented that show the regional mining production levels associated with each commodity, along with a statistical comparison between mining records from the region, the U.S., and the world. The illustrations provide a visual representation of the historical contributions of Montana and Idaho mineral production, which can help identify future trends in the regional mining industry.

The metals and industrial minerals summarized in this report were selected based on the availability of mining production data from U.S. Geological Survey and U.S. Bureau of Mines publications. Some regional commodities show near continuous records of mining production over the last century, while other minerals were only produced occasionally, or have very limited recorded data. Some commodities, such as molybdenum, have a substantial production history in the region though are not included in this report as data are largely withheld from publication. Other important commodities not addressed, such as cobalt, garnet, perlite, vermiculite, and zeolite may be important in terms of future potential for the regional mining industry; however, their historical contributions are either limited or unavailable, and are not useful for identifying industry trends.

In an attempt to explain some of the fluctuations in the rates of production exemplified in the various illustrations, we also include tables and descriptions of noteworthy historical events affecting both the regional and national mining industry for each commodity. These historical events and trends may help to identify causes for some of the more noticeable variances in production rates. However, this historical information should be treated as a starting place only, and is not intended to be a comprehensive account of all major and minor events relating to mining in Idaho and Montana, or the nation. The regional and national mining events are presented as supplemental information to the primary purpose of this report, which is to provide a normalization of mining statistics for the major commodities mined in Idaho and Montana over nearly a century of mining activity. 


\section{Source of information}

The mineral production information was compiled from the state mineral reports in U.S. Bureau of Mines (USBM) Yearbooks published annually from 1905-1996 and from U.S. Geological Survey (USGS) Minerals Yearbooks from 1997 to 2001. The USBM data was published in single volumes from 1905 to 1951, and in multiple volume sets from 1952 until the agency closed in 1996. Mining data for years inclusive of 1952 to 1962 were obtained from Volume III: Area Reports in the USBM yearbooks. Data were obtained for the years of 1963 to 1996 from Area Reports: Domestic, which was published as Volume II or Volume III. In 1997, the USGS assumed the responsibility of publishing yearly mineral and mining statistics. Data from 1997 to 2001 were obtained from USGS Minerals Yearbooks Volume II - Area Reports: Domestic. Digital versions of the USBM mineral yearbooks from 1935-1993 can be found on-line at: http://minerals.usgs.gov/minerals/pubs/usbmmyb.html. The USGS provides on-line Minerals Yearbooks from 1994 to present at: http://minerals.usgs.gov/minerals/pubs/myb.html.

\section{Methods}

For this report, all units of measure were standardized to metric tons (Mt) to facilitate comparison. The conversion of units was necessary because record keeping methods have evolved over the years. For instance, early copper production was first recorded in pounds. In 1948, the units were changed to short tons, and today copper production is recorded in metric tons. The conversion factors used to normalize these data are listed in Appendix 1.

Tables of production for each commodity mined in Idaho and Montana list the production quantity from each state, as well as the quantity from both states combined. In addition to state production, U.S. and world commodity production and the percentage from each that was contributed by the study region is also included in the tables. Years of no recorded production are shown as a leader $\left(_{-}{ }_{-}\right)$, and proprietary data withheld from publication is denoted with the letter "W." Furthermore, the Microsoft ${ }^{\circledR}$ Excel spreadsheet accompanying this report, commodity_production.xls, contains digital versions of the production tables for all of the commodities. Similar to the report tables, all numerical data contained in the spreadsheet are provided in metric tons and share the same conventions, such as "W" for "withheld data" and "n.d." for "no data". Each production table in the spreadsheet is labeled by commodity and is included as a separate worksheet within the Excel file.

Care must be taken to avoid confusion when comparing the figures for regional production to national or world production. All regional mining production numbers are based on crude (raw) mine production; however, national and world mining production will vary by commodity. The figures for U.S. and world production may emphasize resources obtained from only domestic ores, or may represent refined domestic and imported ores. When available, mine production figures for U.S. and world commodities are used to allow for a more pragmatic comparison with regional values. Clarification of the individual methods of reporting is outlined within each commodity section. Further information about specific mineral reporting can be found in Kelly and others (2001). Secondary production, such as recycled scrap metals, is not included in this report.

\section{Illustrations}

Production data are illustrated for every commodity in a series of bar charts. The annual production from Idaho and Montana is illustrated in the first two figures, respectively; combined production from both states is illustrated in the third figure for each commodity. U.S. production data is illustrated in the fourth figure. The annual percentage of the U.S. production and world production from Idaho and Montana combined is illustrated in the fifth and sixth figures, respectively. The seventh figure is 
a line graph illustrating historical price per unit (tons) of the commodity in constant 1998 dollars. Some of these figures are not present for all commodities because information was not always available.

Each illustration is designed with a scale that most efficiently displays the production data. Usually, a y-axis scale was chosen which maximizes the vertical extent of the bars in the graph window. However, for similar illustrations such as the production of a commodity from Idaho, Montana, or the combined state production, a common y-axis scale is used for all three charts in order to allow direct visual comparison between them. The x-axis in each chart represents the range of years $1905-2001$. Bars extending below the $\mathrm{x}$-axis should not be confused as being negative production levels for that year, but instead represent years where data are withheld due to proprietary concerns. There is no numerical value associated with bars below the $\mathrm{x}$-axis as it is only intended as a visual illustration that production has occurred; however, the data is not available. 


\section{Commodities}

\section{Antimony}

Very little antimony was produced in the region prior to the early 1930's (fig. 1). From 1942 until it's closing in 1952, Idaho's Yellow Pine mine yielded the largest antimony production in U.S. history, peaking at over 16,500 metric tons in 1947 (fig. 1A). After World War II, production stabilized at 500 to 1000 metric tons per year. From 1953 until 2000, the primary, and often sole, antimony producer in Idaho was the Sunshine mine in the Coeur d'Alene mining district. Data withheld in the USBM minerals yearbook during this period was obtained from Sunshine Annual Reports. Montana has never been a significant producer of antimony (fig. 1B). The small amounts that have been recovered were from U.S. Antimony's Babbitt mine or as by-products from other mining activities (Kelly and others, 2001). Though domestic mine production of antimony in the U.S. has been relatively minor in recent years, the production figures show a close relationship with production from Idaho (fig. 1D). Beginning in the early 1930's, regional production proved to be critical to the national supply, as Idaho's mines have often contributed 90100 percent of national production of antimony (fig. 1E). As a global antimony producer, the Idaho alone supplied nearly 50 percent of the world's antimony in 1945 and 1946 (fig. 1F). In the 50 years following the closure of the Yellow Pine mine, Idaho and Montana combined have averaged about 1 percent of world antimony production.

Table 1. Selected events affecting regional antimony production

\begin{tabular}{lll}
\hline Year & Event & Reference \\
\hline 1941 & $\begin{array}{l}\text { Yellow Pine mine opens in Idaho and becomes the largest } \\
\text { antimony producer in the US during WWII }\end{array}$ & Miller, M.H., 1973 \\
1952 & Yellow Pine mine closes & Miller, M.H., 1973 \\
1953 & Sunshine electrolytic plant reactivated & this study \\
1970 & US Antimony mine opened & this study \\
1972 & Sunshine mine fire curtailed production & this study \\
1980 & Sunshine production curtailed by strike & this study \\
1983 & US Antimony's Thompson Falls Babbitt mine closed in favor of & this study \\
& importing antimony ore for smelting. & \\
\hline
\end{tabular}

Table 2. Selected events affecting U.S. antimony production

\begin{tabular}{|c|c|c|}
\hline Year & Event & Reference \\
\hline 1914-1918 & WWI & \\
\hline 1939-1945 & WWII & \\
\hline 1970 & Antimony-based flame retardants developed & Kramer, undated \\
\hline 1994-1995 & $\begin{array}{l}\text { Flooding in China's mining districts causes severe short supply of } \\
\text { antimony }\end{array}$ & Carlin, 1999 \\
\hline
\end{tabular}



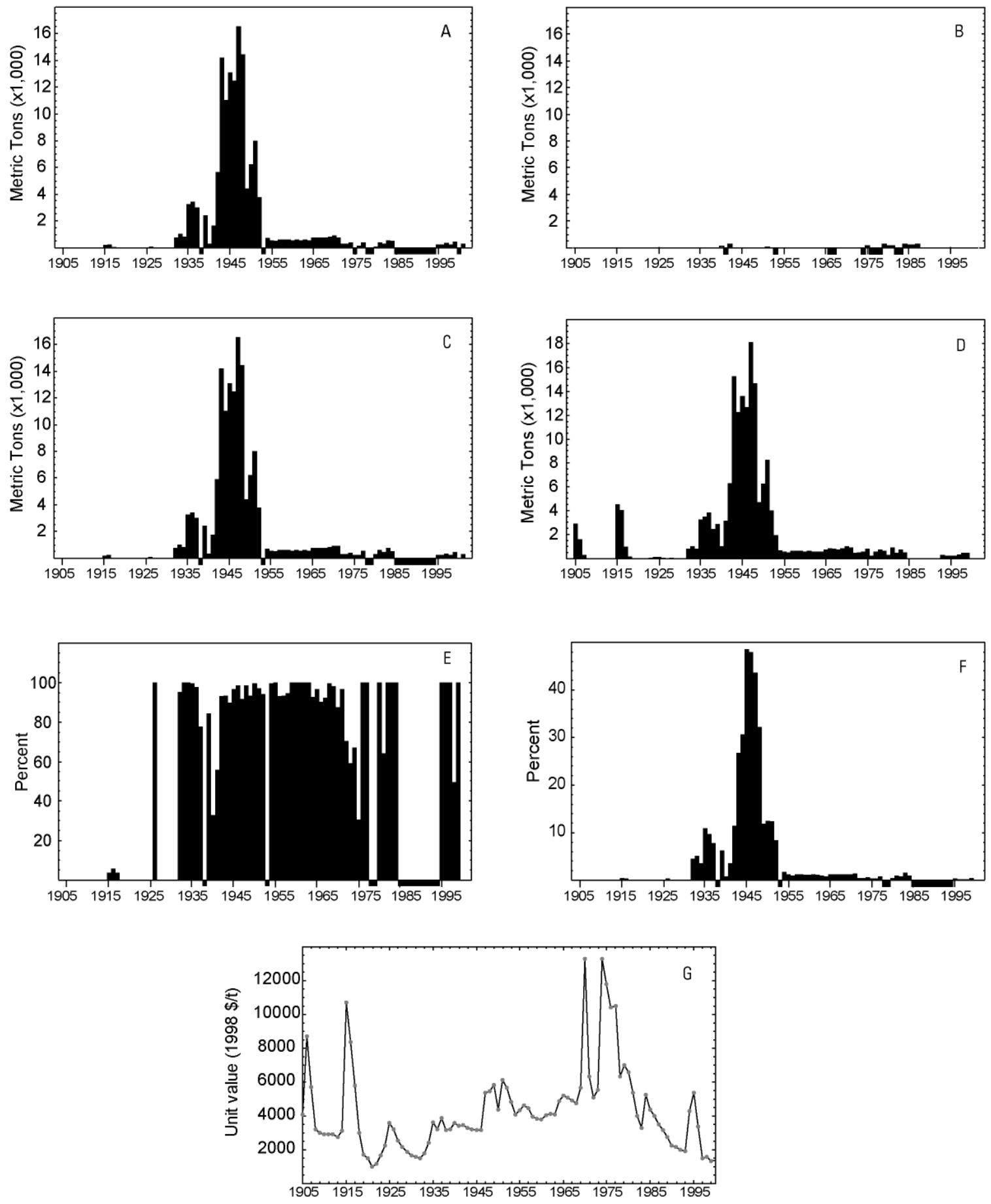

Figure 1. Antimony production information from 1905-2001. A, annual production from Idaho. B, annual production from Montana. C, Idaho and Montana combined production. D, annual U.S. antimony production of ores and concentrates. $\mathrm{E}$, the proportion of regional production to U.S. mine production. $F$, the proportion of regional production to world mine production. $\mathrm{G}$, the historical value of antimony in constant 1998 dollars. Bars extending below the x-axis represent data withheld from publication. 
Table 3. Antimony production from Idaho and Montana, the U.S., and the world, $1905-2001$

[The table also summarizes the proportions of Idaho and Montana production relative to the U.S. and the world as well as the price for antimony in constant 1998 dollars. Production figures for the U.S. reflect mine production of ores and concentrates; world production figures represent mine production in terms of antimony content. Data for state, US, and world production are given in metric tons; proportions of U.S. and world production by Idaho and Montana are given in percent; antimony value is in US dollars. W, withheld; n.d., no data; leaders ( $\_$- ), no production]

Production

\begin{tabular}{|c|c|c|c|c|c|c|c|c|}
\hline \multirow[b]{2}{*}{ Year } & \multicolumn{5}{|c|}{ Production } & \multicolumn{2}{|c|}{ production to: } & \multirow[b]{2}{*}{$\begin{array}{r}\text { Unit } \\
\text { value } \\
(1998 \$ / t)\end{array}$} \\
\hline & Idaho & Montana & $\begin{array}{r}\text { Idaho and } \\
\text { Montana } \\
\text { combined }\end{array}$ & US & World & US & World & \\
\hline 1905 & - - & -- & - - & 2,939 & 8,000 & 0 & 0 & 4,100 \\
\hline 1906 & -- & -- & -- & 1,602 & 14,500 & 0 & 0 & 8,700 \\
\hline 1907 & _- & -- & -- & 318 & 15,000 & 0 & 0 & 5,700 \\
\hline 1908 & -- & -- & -- & -- & 16,000 & 0 & 0 & 3,200 \\
\hline 1909 & - - & _- & _- & -- & 15,000 & 0 & 0 & 3,000 \\
\hline 1910 & _- & _- & _- & _- & 15,000 & 0 & 0 & 2,900 \\
\hline 1911 & $\ldots$ & $\ldots$ & $\ldots$ & -- & 15,500 & 0 & 0 & 2,900 \\
\hline 1912 & -- & -- & -- & -- & 24,200 & 0 & 0 & 2,900 \\
\hline 1913 & -- & -- & -- & -- & 24,500 & 0 & 0 & 2,730 \\
\hline 1914 & - - & - - & -- & -- & 23,600 & 0 & 0 & 3,130 \\
\hline 1915 & 175 & -- & 175 & 4,536 & 43,200 & 3.86 & 0.41 & 10,700 \\
\hline 1916 & 235 & $\ldots$ & 235 & 4,082 & 81,600 & 5.76 & 0.29 & 8,360 \\
\hline 1917 & 35 & -- & 35 & 961 & 57,200 & 3.68 & 0.06 & 5,800 \\
\hline 1918 & -- & -- & -- & 172 & 30,800 & 0 & 0 & 3,010 \\
\hline 1919 & -- & -- & -- & -- & 11,800 & 0 & 0 & 1,700 \\
\hline 1920 & -- & -- & -- & -- & 29,000 & 0 & 0 & 1,520 \\
\hline 1921 & -- & -- & - - & -- & 18,300 & 0 & 0 & 991 \\
\hline 1922 & -- & -- & -- & 18 & 18,900 & 0 & 0 & 1,170 \\
\hline 1923 & -- & -- & -- & 36 & 17,600 & 0 & 0 & 1,660 \\
\hline 1924 & -- & -- & -- & 103 & 17,500 & 0 & 0 & 2,270 \\
\hline 1925 & -- & -- & -- & 112 & 25,500 & 0 & 0 & 3,570 \\
\hline 1926 & 90 & -- & 90 & 90 & 29,000 & 100.00 & 0.31 & 3,220 \\
\hline 1927 & -- & -- & -- & -- & 28,000 & 0 & 0 & 2,530 \\
\hline 1928 & -- & -- & -- & 78 & 28,500 & 0 & 0 & 2,160 \\
\hline 1929 & -- & -- & -- & -- & 31,600 & 0 & 0 & 1,880 \\
\hline 1930 & -- & -- & -- & -- & 23,600 & 0 & 0 & 1,660 \\
\hline 1931 & -- & -- & -- & -- & 15,600 & 0 & 0 & 1,580 \\
\hline 1932 & 778 & -- & 778 & 816 & 17,300 & 95.33 & 4.50 & 1,480 \\
\hline 1933 & 1,028 & -- & 1,028 & 1,028 & 20,200 & 100.00 & 5.09 & 1,810 \\
\hline 1934 & 814 & -- & 814 & 814 & 22,600 & 100.00 & 3.60 & 2,400 \\
\hline 1935 & 3,268 & -- & 3,268 & 3,280 & 29,800 & 99.61 & 10.97 & 3,630 \\
\hline 1936 & 3,436 & -- & 3,436 & 3,508 & 35,300 & 97.93 & 9.73 & 3,190 \\
\hline 1937 & 2,989 & -- & 2,989 & 3,856 & 38,600 & 77.53 & 7.74 & 3,860 \\
\hline 1938 & W & -- & W & 2,477 & 33,900 & W & W & 3,160 \\
\hline 1939 & 2,429 & -- & 2,429 & 2,879 & 38,800 & 84.34 & 6.26 & 3,200 \\
\hline 1940 & 274 & 63 & 337 & 1,020 & 46,300 & 33.01 & 0.73 & 3,590 \\
\hline 1941 & 1,654 & 103 & 1,757 & 3,139 & 49,000 & 55.97 & 3.59 & 3,420 \\
\hline 1942 & 5,647 & 254 & 5,901 & 6,332 & 51,400 & 93.19 & 11.48 & 3,440 \\
\hline
\end{tabular}

Proportion of Idaho and Montana production to: nit 100 00 200 900 900 730 360 , 800 ,520 991 70 60 270 570 530 160 ,880 ,660 ,580 ,480 630 , 160 ,200 , 440 


\begin{tabular}{|c|c|c|c|c|c|c|c|c|}
\hline 1943 & 14,205 & -- & 14,205 & 15,227 & 53,200 & 93.29 & 26.70 & 3,310 \\
\hline 1944 & 11,007 & -- & 11,007 & 12,248 & 36,000 & 89.87 & 30.58 & 3,220 \\
\hline 1945 & 13,123 & -- & 13,123 & 13,577 & 27,000 & 96.65 & 48.60 & 3,160 \\
\hline 1946 & 12,458 & -- & 12,458 & 12,666 & 26,000 & 98.35 & 47.91 & 3,180 \\
\hline 1947 & 16,564 & -- & 16,564 & 18,126 & 38,000 & 91.38 & 43.59 & 5,390 \\
\hline 1948 & 14,462 & -- & 14,462 & 14,670 & 45,000 & 98.58 & 32.14 & 5,470 \\
\hline 1949 & 4,389 & -- & 4,389 & 4,705 & 37,000 & 93.29 & 11.86 & 5,840 \\
\hline 1950 & 6,231 & -- & 6,231 & 6,249 & 50,000 & 99.71 & 12.46 & 4,380 \\
\hline 1951 & 7,988 & 26 & 8,014 & 8,256 & 65,000 & 97.08 & 12.33 & 6,130 \\
\hline 1952 & 3,786 & -- & 3,786 & 4,023 & 44,500 & 94.11 & 8.51 & 5,680 \\
\hline 1953 & W & W & W & 1,960 & 33,600 & W & W & 4,820 \\
\hline 1954 & 693 & -- & 693 & 695 & 39,900 & 99.74 & 1.74 & 4,070 \\
\hline 1955 & 574 & -- & 574 & 574 & 46,300 & 100.00 & 1.24 & 4,330 \\
\hline 1956 & 498 & -- & 498 & 535 & 53,500 & 93.05 & 0.93 & 4,620 \\
\hline 1957 & 602 & -- & 602 & 644 & 50,800 & 93.52 & 1.19 & 4,470 \\
\hline 1958 & 614 & -- & 614 & 650 & 46,300 & 94.55 & 1.33 & 3,960 \\
\hline 1959 & 615 & -- & 615 & 615 & 53,300 & 100.00 & 1.15 & 3,850 \\
\hline 1960 & 576 & -- & 576 & 576 & 53,300 & 100.00 & 1.08 & 3,790 \\
\hline 1961 & 625 & -- & 625 & 625 & 51,900 & 100.00 & 1.20 & 4,060 \\
\hline 1962 & 572 & -- & 572 & 572 & 53,700 & 100.00 & 1.07 & 4,120 \\
\hline 1963 & 585 & -- & 585 & 585 & 58,000 & 100.00 & 1.01 & 4,080 \\
\hline 1964 & 531 & -- & 531 & 573 & 63,000 & 92.62 & 0.84 & 4,890 \\
\hline 1965 & 742 & -- & 742 & 767 & 63,000 & 96.75 & 1.18 & 5,210 \\
\hline 1966 & 757 & -- & 757 & 841 & 61,400 & 89.96 & 1.23 & 5,080 \\
\hline 1967 & 747 & -- & 747 & 809 & 58,400 & 92.29 & 1.28 & 4,930 \\
\hline 1968 & 774 & -- & 774 & 777 & 61,500 & 99.59 & 1.26 & 4,740 \\
\hline 1969 & 836 & -- & 836 & 851 & 66,200 & 98.29 & 1.26 & 5,650 \\
\hline 1970 & 901 & W & 901 & 1,030 & 70,000 & 87.46 & 1.29 & 13,300 \\
\hline 1971 & 777 & 122 & 900 & 930 & 64,100 & 96.77 & 1.40 & 6,320 \\
\hline 1972 & 313 & W & 313 & 444 & 68,100 & 70.49 & 0.46 & 5,070 \\
\hline 1973 & 292 & W & 292 & 494 & 69,300 & 59.13 & 0.42 & 5,540 \\
\hline 1974 & 404 & W & 404 & 600 & 70,500 & 67.28 & 0.57 & 13,300 \\
\hline 1975 & W & 248 & 248 & 804 & 67,900 & 30.80 & 0.36 & 11,800 \\
\hline 1976 & 121 & 136 & 257 & 257 & 69,200 & 100.00 & 0.37 & 10,400 \\
\hline 1977 & 405 & 149 & 553 & 553 & 72,200 & 100.00 & 0.77 & 10,500 \\
\hline 1978 & W & W & $\mathrm{W}$ & 724 & 68,800 & W & W & 6,350 \\
\hline 1979 & W & W & W & 655 & 71,900 & $\mathrm{~W}$ & W & 6,990 \\
\hline 1980 & 75 & 236 & 311 & 311 & 67,200 & 100.00 & 0.46 & 6,590 \\
\hline 1981 & 392 & 194 & 586 & 914 & 59,200 & 64.12 & 0.99 & 5,380 \\
\hline 1982 & 267 & 190 & 456 & 456 & 53,800 & 100.00 & 0.85 & 3,990 \\
\hline 1983 & 531 & 230 & 760 & 760 & 48,400 & 100.00 & 1.57 & 3,290 \\
\hline 1984 & 505 & -- & 505 & 505 & 53,400 & 100.00 & 0.95 & 5,230 \\
\hline 1985 & W & -- & W & -- & 55,000 & W & W & 4,380 \\
\hline 1986 & W & -- & W & -- & 59,900 & W & W & 4,000 \\
\hline 1987 & W & -- & W & -- & 56,100 & W & W & 3,510 \\
\hline 1988 & W & -- & W & -- & 64,400 & W & W & 3,160 \\
\hline 1989 & W & -- & W & -- & 68,400 & W & W & 2,740 \\
\hline 1990 & W & -- & W & -- & 60,400 & W & W & 2,250 \\
\hline 1991 & W & -- & W & -- & 64,700 & W & W & 2,170 \\
\hline 1992 & W & -- & W & -- & 76,000 & W & W & 2,020 \\
\hline
\end{tabular}




\begin{tabular}{rrrrrrrrr}
1993 & W & -- & W & 266 & 73,000 & W & W & 1,920 \\
1994 & W & -- & W & 215 & 106,000 & W & W & 4,310 \\
1995 & 262 & -- & 262 & 262 & 103,000 & 100.00 & 0.25 & 5,380 \\
1996 & 242 & -- & 242 & 242 & 156,000 & 100.00 & 0.16 & 3,370 \\
1997 & 356 & -- & 356 & 356 & 155,000 & 100.00 & 0.23 & 1,510 \\
1998 & 242 & -- & 242 & 489 & 117,000 & 49.49 & 0.21 & 1,580 \\
1999 & 450 & -- & 450 & 450 & 108,000 & 100.00 & 0.42 & 1,350 \\
2000 & W & -- & W & -- & 118,000 & 0 & 0 & 1,360 \\
2001 & 300 & -- & 300 & -- & n.d & n.d & n.d & n.d \\
\hline
\end{tabular}




\section{Clay}

Clay production values are reported in the amount of domestic material sold or used by producers, however abundant clay construction material was not included in this data before 1943 (Kelly and others, 2001). Prior to this time, the scanty clay production values from Idaho that were not withheld amounted to mere hundreds of tons (fig. 2A). Larger production years in Idaho followed, however they did not reach the levels of Montana. From 1970 until 1986, Montana saw an increase in clay production that eventually reached a maximum of nearly 600,000 metric tons (fig. 2B). The combined production from Idaho and Montana also shows the change in clay production reporting in the mid-1940s, as does the U.S. clay production, though the increase on the national scale was much more dramatic. Historically, annual clay mining in Idaho and Montana combined is less than 0.5 percent of what the U.S. has produced as a whole, although production in 1980 and 1981 spiked to approximately 1.4 percent (fig. 2E). Data for world clay production were unavailable.
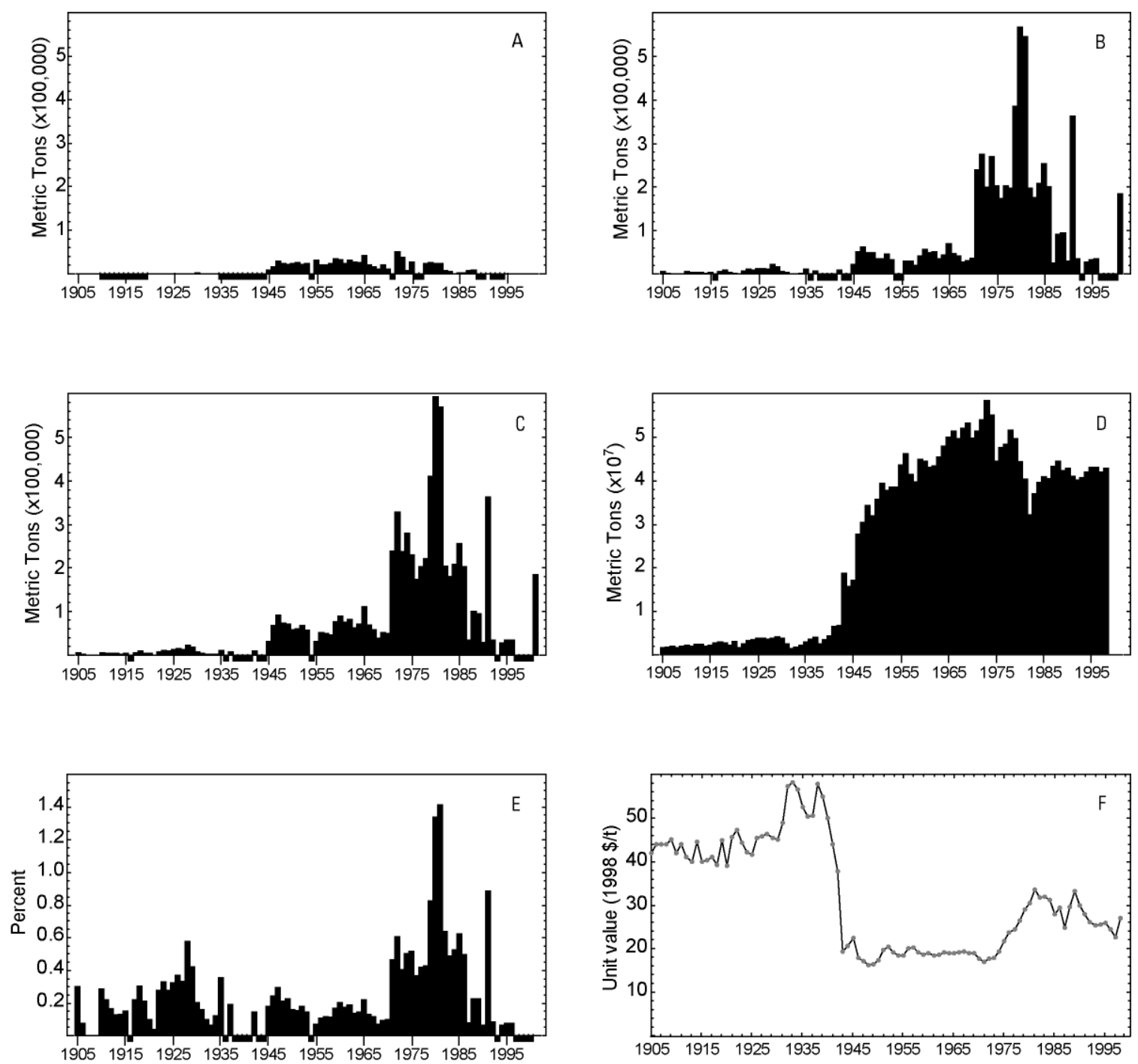

Figure 2. Clay production information from 1905-2001. A, annual production from Idaho. B, annual production from Montana. C, Idaho and Montana combined production. D, annual U.S. clay production in millions of metric tons. E, the proportion of regional production to U.S. mine production. F, the historical value of clay in constant 1998 dollars. Bars extending below the $\mathrm{x}$-axis represent data withheld from publication. 
Table 4. Clay production from Idaho, Montana, and the U.S., 1905-2001

[The table also summarizes the proportions of Idaho and Montana production relative to the US as well as the price for clay in constant 1998 dollars. Data for state and US production are given in metric tons; proportion of US production by Idaho and Montana are given in percent; clay value is in US dollars. W, withheld; n.d., no data; leaders ( $\_$), no production]

\begin{tabular}{|c|c|c|c|c|c|c|}
\hline \multirow[b]{2}{*}{ Year } & \multicolumn{4}{|c|}{ Production } & \multirow{2}{*}{$\begin{array}{r}\text { Proportion of Idaho and } \\
\text { Montana production to: } \\
\text { US }\end{array}$} & \multirow[b]{2}{*}{$\begin{array}{r}\text { Clay value } \\
(1998 \text { \$/t })\end{array}$} \\
\hline & Idaho & Montana & $\begin{array}{l}\text { Idaho and } \\
\text { Montana } \\
\text { combined }\end{array}$ & US & & \\
\hline 1905 & -- & 5,031 & 5,031 & $1,661,000$ & 0.30 & 42.00 \\
\hline 1906 & -- & 1,465 & 1,465 & $1,868,000$ & 0.08 & 44.00 \\
\hline 1907 & -- & -- & -- & $2,011,000$ & -- & 44.00 \\
\hline 1908 & -- & -- & -- & $1,591,000$ & -- & 44.00 \\
\hline 1909 & -- & -- & -- & $1,990,000$ & -- & 45.00 \\
\hline 1910 & W & 6,360 & 6,360 & $2,197,000$ & 0.29 & 42.00 \\
\hline 1911 & $\mathrm{~W}$ & 4,456 & 4,456 & $2,017,000$ & 0.22 & 44.00 \\
\hline 1912 & W & 3,810 & 3,810 & $2,325,000$ & 0.16 & 41.00 \\
\hline 1913 & $\mathrm{~W}$ & 3,094 & 3,094 & $2,436,000$ & 0.13 & 40.00 \\
\hline 1914 & W & 2,702 & 2,702 & $2,042,000$ & 0.13 & 44.50 \\
\hline 1915 & W & 3,329 & 3,329 & $2,200,000$ & 0.15 & 40.00 \\
\hline 1916 & $\mathrm{~W}$ & W & W & $2,722,000$ & W & 40.30 \\
\hline 1917 & W & 6,426 & 6,426 & $2,925,000$ & 0.22 & 41.10 \\
\hline 1918 & W & 8,544 & 8,544 & $2,777,000$ & 0.31 & 39.30 \\
\hline 1919 & W & 4,606 & 4,606 & $2,160,000$ & 0.21 & 44.90 \\
\hline 1920 & 792 & 2,342 & 3,134 & $3,091,000$ & 0.10 & 39.00 \\
\hline 1921 & 178 & 474 & 652 & $1,680,000$ & 0.04 & 45.60 \\
\hline 1922 & 357 & 6,680 & 7,037 & $2,528,000$ & 0.28 & 47.20 \\
\hline 1923 & 121 & 10,545 & 10,666 & $3,251,000$ & 0.33 & 44.40 \\
\hline 1924 & 517 & 9,319 & 9,836 & $3,510,000$ & 0.28 & 42.10 \\
\hline 1925 & 194 & 12,271 & 12,465 & $3,844,000$ & 0.32 & 41.50 \\
\hline 1926 & 516 & 13,595 & 14,111 & $3,811,000$ & 0.37 & 45.50 \\
\hline 1927 & 433 & 11,981 & 12,414 & $3,732,000$ & 0.33 & 45.80 \\
\hline 1928 & 448 & 22,265 & 22,714 & $3,913,000$ & 0.58 & 46.40 \\
\hline 1929 & 559 & 17,346 & 17,904 & $4,230,000$ & 0.42 & 45.50 \\
\hline 1930 & 1,421 & 6,563 & 7,983 & $3,900,000$ & 0.20 & 45.10 \\
\hline 1931 & 235 & 3,877 & 4,112 & $2,547,000$ & 0.16 & 48.90 \\
\hline 1932 & 414 & 1,088 & 1,501 & $1,470,000$ & 0.10 & 57.30 \\
\hline 1933 & 229 & 991 & 1,219 & $1,873,000$ & 0.07 & 58.20 \\
\hline 1934 & 256 & 2,427 & 2,683 & $2,184,000$ & 0.12 & 56.50 \\
\hline 1935 & W & 10,291 & 10,291 & $2,859,000$ & 0.36 & 52.50 \\
\hline 1936 & W & W & W & $3,641,000$ & W & 50.40 \\
\hline 1937 & W & 7,843 & 7,843 & $4,049,000$ & 0.19 & 50.50 \\
\hline 1938 & W & W & W & $2,632,000$ & W & 57.80 \\
\hline 1939 & $\mathrm{~W}$ & W & W & $3,563,000$ & W & 54.90 \\
\hline 1940 & $\mathrm{~W}$ & W & W & $4,398,000$ & W & 50.00 \\
\hline
\end{tabular}




\begin{tabular}{|c|c|c|c|c|c|c|}
\hline 1941 & W & W & W & $6,555,000$ & W & 43.90 \\
\hline 1942 & W & 9,914 & 9,914 & $6,847,000$ & 0.14 & 37.70 \\
\hline 1943 & W & W & W & $18,886,000$ & W & 19.30 \\
\hline 1944 & W & W & $\mathrm{W}$ & $15,690,000$ & W & 20.60 \\
\hline 1945 & 8,741 & 22,479 & 31,219 & $17,168,000$ & 0.18 & 22.40 \\
\hline 1946 & 16,961 & 51,187 & 68,148 & $27,727,000$ & 0.25 & 17.90 \\
\hline 1947 & 28,958 & 61,610 & 90,568 & $30,481,000$ & 0.30 & 17.20 \\
\hline 1948 & 23,587 & 49,896 & 73,483 & $34,226,000$ & 0.21 & 16.30 \\
\hline 1949 & 22,680 & 48,989 & 71,669 & $31,888,000$ & 0.22 & 16.40 \\
\hline 1950 & 23,587 & 34,474 & 58,061 & $35,726,000$ & 0.16 & 17.40 \\
\hline 1951 & 25,657 & 35,590 & 61,247 & $39,386,000$ & 0.16 & 19.70 \\
\hline 1952 & 21,349 & 46,543 & 67,892 & $37,801,000$ & 0.18 & 20.40 \\
\hline 1953 & 23,795 & 33,561 & 57,356 & $38,488,000$ & 0.15 & 19.10 \\
\hline 1954 & W & W & W & $38,560,000$ & W & 18.50 \\
\hline 1955 & 31,752 & W & 31,752 & $43,640,000$ & 0.07 & 18.40 \\
\hline 1956 & 20,866 & 29,938 & 50,803 & $46,061,000$ & 0.11 & 20.10 \\
\hline 1957 & 20,866 & 29,030 & 49,896 & $41,388,000$ & 0.12 & 20.20 \\
\hline 1958 & 24,494 & 20,866 & 45,360 & $39,689,000$ & 0.11 & 19.20 \\
\hline 1959 & 35,381 & 41,731 & 77,112 & $44,800,000$ & 0.17 & 18.60 \\
\hline 1960 & 32,659 & 57,154 & 89,813 & $44,515,000$ & 0.20 & 18.90 \\
\hline 1961 & 24,494 & 49,896 & 74,390 & $42,991,000$ & 0.17 & 18.50 \\
\hline 1962 & 31,752 & 50,803 & 82,555 & $43,361,000$ & 0.19 & 18.70 \\
\hline 1963 & 28,123 & 34,474 & 62,597 & $45,482,000$ & 0.14 & 19.10 \\
\hline 1964 & 26,309 & 44,453 & 70,762 & $48,033,000$ & 0.15 & 18.90 \\
\hline 1965 & 42,638 & 68,947 & 111,586 & $50,009,000$ & 0.22 & 19.00 \\
\hline 1966 & 20,866 & 48,082 & 68,947 & $51,449,000$ & 0.13 & 19.20 \\
\hline 1967 & 17,237 & 41,731 & 58,968 & $49,590,000$ & 0.12 & 19.40 \\
\hline 1968 & 10,886 & 27,216 & 38,102 & $52,025,000$ & 0.07 & 18.90 \\
\hline 1969 & 20,866 & 30,845 & 51,710 & $53,246,000$ & 0.10 & 18.90 \\
\hline 1970 & 11,794 & 37,195 & 48,989 & $49,762,000$ & 0.10 & 17.72 \\
\hline 1971 & W & 239,501 & 239,501 & $51,407,000$ & 0.47 & 16.99 \\
\hline 1972 & 51,710 & 275,789 & 327,499 & $53,938,000$ & 0.61 & 17.65 \\
\hline 1973 & 38,102 & 198,677 & 236,779 & $58,378,000$ & 0.41 & 17.83 \\
\hline 1974 & 8,165 & 270,346 & 278,510 & $55,153,000$ & 0.50 & 19.27 \\
\hline 1975 & 27,216 & 202,306 & 229,522 & $44,495,000$ & 0.52 & 21.72 \\
\hline 1976 & W & 174,182 & 174,182 & $47,527,000$ & 0.37 & 23.80 \\
\hline 1977 & W & 203,213 & 203,213 & $48,259,000$ & 0.42 & 24.48 \\
\hline 1978 & 24,494 & 196,862 & 221,357 & $51,548,000$ & 0.43 & 26.54 \\
\hline 1979 & 25,402 & 384,653 & 410,054 & $49,613,000$ & 0.83 & 29.04 \\
\hline 1980 & 24,494 & 567,907 & 592,402 & $44,262,000$ & 1.34 & 30.55 \\
\hline 1981 & 23,587 & 545,227 & 568,814 & $40,260,000$ & 1.41 & 33.55 \\
\hline 1982 & 7,258 & 197,770 & 205,027 & $32,064,000$ & 0.64 & 31.80 \\
\hline 1983 & 5,443 & 175,997 & 181,440 & $37,066,000$ & 0.49 & 31.88 \\
\hline 1984 & 907 & 207,749 & 208,656 & $39,646,000$ & 0.53 & 31.16 \\
\hline 1985 & 1,814 & 253,109 & 254,923 & $40,908,000$ & 0.62 & 27.90 \\
\hline 1986 & 1,814 & 201,398 & 203,213 & $40,580,000$ & 0.50 & 29.44 \\
\hline 1987 & 8,114 & 26,199 & 34,313 & $43,234,000$ & 0.08 & 24.83 \\
\hline 1988 & 8,519 & 91,802 & 100,321 & $44,515,000$ & 0.23 & 29.59 \\
\hline
\end{tabular}




\begin{tabular}{lrrrrrr}
1989 & W & 95,743 & 95,743 & $42,254,000$ & 0.23 & 33.19 \\
1990 & $\mathrm{~W}$ & 29,741 & 29,741 & $42,900,000$ & 0.07 & 29.89 \\
1991 & 967 & 362,635 & 363,602 & $41,000,000$ & 0.89 & 27.87 \\
1992 & $\mathrm{~W}$ & 35,000 & 35,000 & $40,200,000$ & 0.09 & 26.06 \\
1993 & $\mathrm{~W}$ & $\mathrm{~W}$ & $\mathrm{~W}$ & $40,700,000$ & $\mathrm{~W}$ & 25.32 \\
1994 & $\mathrm{~W}$ & 28,000 & 28,000 & $42,000,000$ & 0.07 & 25.57 \\
1995 & 1,000 & 33,000 & 34,000 & $43,100,000$ & 0.08 & 25.86 \\
1996 & -- & 34,000 & 34,000 & $43,100,000$ & 0.08 & 24.50 \\
1997 & -- & $\mathrm{W}$ & $\mathrm{W}$ & $41,989,000$ & $\mathrm{~W}$ & 22.59 \\
1998 & -- & $\mathrm{W}$ & $\mathrm{W}$ & $42,934,000$ & $\mathrm{~W}$ & 27.02 \\
1999 & -- & $\mathrm{W}$ & $\mathrm{W}$ & n.d. & n.d. & n.d. \\
2000 & -- & $\mathrm{W}$ & $\mathrm{W}$ & n.d. & n.d. & n.d. \\
2001 & -- & 185,000 & 185,000 & n.d. & n.d. & n.d. \\
\hline
\end{tabular}




\section{Copper}

Although Idaho has occasionally produced around 5000 metric tons of copper per year (fig. 3A), its production is relatively insignificant compared to Montana, specifically the Butte area. Though small yearly fluctuations in production from Montana existed, vein mining at Butte produced roughly 100,000 to 120,000 metric tons per year and reached a peak in 1916 of 160,000 (fig. 3B). The dominance of regional copper production coming from Montana is exemplified in figure $3 \mathrm{C}$ where the combined production from Idaho and Montana is nearly identical to the production values in figure 3B. In the early 20th century, Idaho and Montana contributed nearly 35 percent of the national copper production (fig. 3E). However, with the development of open-pit mining of porphyry deposits elsewhere in the U.S. and contribution to a steady growing trend in national copper production, the proportion from Idaho and Montana declined to where it contributed less than five percent of the national production in the late 1990s (Cox and others, 1973). Figure $3 F$ shows a similar decreasing trend in the role of Idaho and Montana in world copper production when, in 1905, they combined for more than 20 percent of world production, but today are less than 1 percent. When vein production began waning in the early to mid-1940s, Butte began transitioning to new methods of extracting ore. Block caving saw greater use in the early 1950s when the Kelley Project came on-line; however by the late 1950s block caving was beginning to be replaced with open pit mining. The Berkeley Pit was started in 1955, which gave the region a boost that persisted through the early 1980's (fig. 3B), though Montana only accounted for about 7 percent of the U.S. copper production (fig. 3E) and 2 to 2.5 percent of world production (fig. $3 F$ ).

Table 5. Selected events affecting regional copper production

\begin{tabular}{|c|c|c|}
\hline Date & Event & Reference \\
\hline $1907-1908$ & Drop in copper prices force layoffs and shutdowns & Everett, 2002 \\
\hline 1914 & Rioting at Miner's Union Day parade in Butte & Everett, 2002 \\
\hline 1917 & $\begin{array}{l}\text { Fire at Granite Mountain mine killed } 168 \text { men, } \\
\text { workers strike in response }\end{array}$ & Everett, 2002 \\
\hline 1919 & Strike over $\$ 1 /$ day wage cuts. & Everett, 2002 \\
\hline Apr. 1921 - Jan. 1922 & $\begin{array}{l}\text { Butte mines shut down because of the low price of } \\
\text { copper }\end{array}$ & Everett, 2002 \\
\hline Summer 1934 & Butte miners strike & Everett, 2002 \\
\hline 1946 & 6-month strike & Edelstein, 1999 \\
\hline $1955-82$ & Butte's Berkeley Pit in operation & $\begin{array}{l}\text { Montana Mining Properties, unpub. } \\
\text { data, } 2003\end{array}$ \\
\hline Aug. 1959 - Feb. 1960 & Butte strike lasting 181 days & Everett, 2002 \\
\hline July 1967 - Mar. 1968 & Longest strike in Butte's history $-81 / 2$ months & Everett, 2002 \\
\hline 1977 & Butte strike & Everett, 2002 \\
\hline 1980 & $41 / 2$ month strike in Butte & Everett, 2002 \\
\hline 1980 & Anaconda smelter closes & Everett, 2002 \\
\hline 1982-1993 & Montana's Troy mine years of operation & this study \\
\hline 1985 & $\begin{array}{l}\text { Montana Resources acquires Continental Pit, } \\
\text { production values withheld }\end{array}$ & $\begin{array}{l}\text { Montana Mining Properties, unpub. } \\
\text { data, } 2003\end{array}$ \\
\hline 1986 & East Berkeley Pit reopened by Montana Resources & $\begin{array}{l}\text { Montana Mining Properties, unpub. } \\
\text { data, } 2003\end{array}$ \\
\hline 2000 & Continental mine suspends operations & this study \\
\hline
\end{tabular}

Table 6. Selected events affecting U.S. copper production

\begin{tabular}{lll} 
Date & Event & Reference \\
\hline 1906 & Large-scale open-pit mining developed on porphyry deposits & Cox and others, 1973 \\
1906 & Froth flotation comes into widespread use & Kramer, undated \\
1913 & Introduction of selective flotation process & Cox and others, 1973 \\
$1914-18$ & WWI - high demand &
\end{tabular}




$\begin{array}{lll}1921 & \text { Economic recession } & \text { Cox and others, } 1973 \\ 1929 & \text { Panic buying } & \text { Cox and others, } 1973 \\ 1930-39 & \text { Great Depression } & \\ 1939-45 & \text { WWII }- \text { high demand } & \\ 1954-56 & \text { Boom after Korean War } & \text { Cox and others, } 1973 \\ 1968 & \text { Electrowinning process to recover copper begins } & \text { Kramer, undated } \\ 1970-73 & \text { High demand on account of the Vietnam War } & \text { Edelstein, } 1999 \\ 1976 & \text { First flash copper smelter commissioned in U.S. } & \text { Kramer, undated } \\ 1982-86 & \text { Cutback in capacity at U.S. mines; production sharply curtailed } & \text { Edelstein, 1999 }\end{array}$

The USBM lists copper production data from Montana as "withheld" from 1985 to 2000. Most of the copper production in Montana came from the ore deposits at Butte. After the privately owned Montana Resources acquired the Continental deposit in 1985, copper production data were no longer published in the state commodity reports. The limited amount of production data found in ASARCO annual reports is summarized in table 7. From 2000 to 2003, mining was suspended at the Continental mine because of high energy costs. Recorded production in the ASARCO annual reports is similar to the last reported value for copper in Montana in 1985.

Table 7. Copper production, in metric tons, from the Continental mine, 1995 to 1999 (ASARC0, 1999)

\begin{tabular}{lccccc}
\hline & 1995 & 1996 & 1997 & 1998 & 1999 \\
\cline { 2 - 6 } & & & & & \\
$\begin{array}{l}\text { Montana Resources } \\
\text { (Continental deposit) }\end{array}$ & 51,170 & 47,540 & 41,500 & 44,600 & 38,700 \\
\hline
\end{tabular}

Mining activities began in Montana in the early 1880's; however copper production data for the region prior to 1905 is not included in this report. Figure 4 shows a non-detailed line chart illustrating early copper production values. 

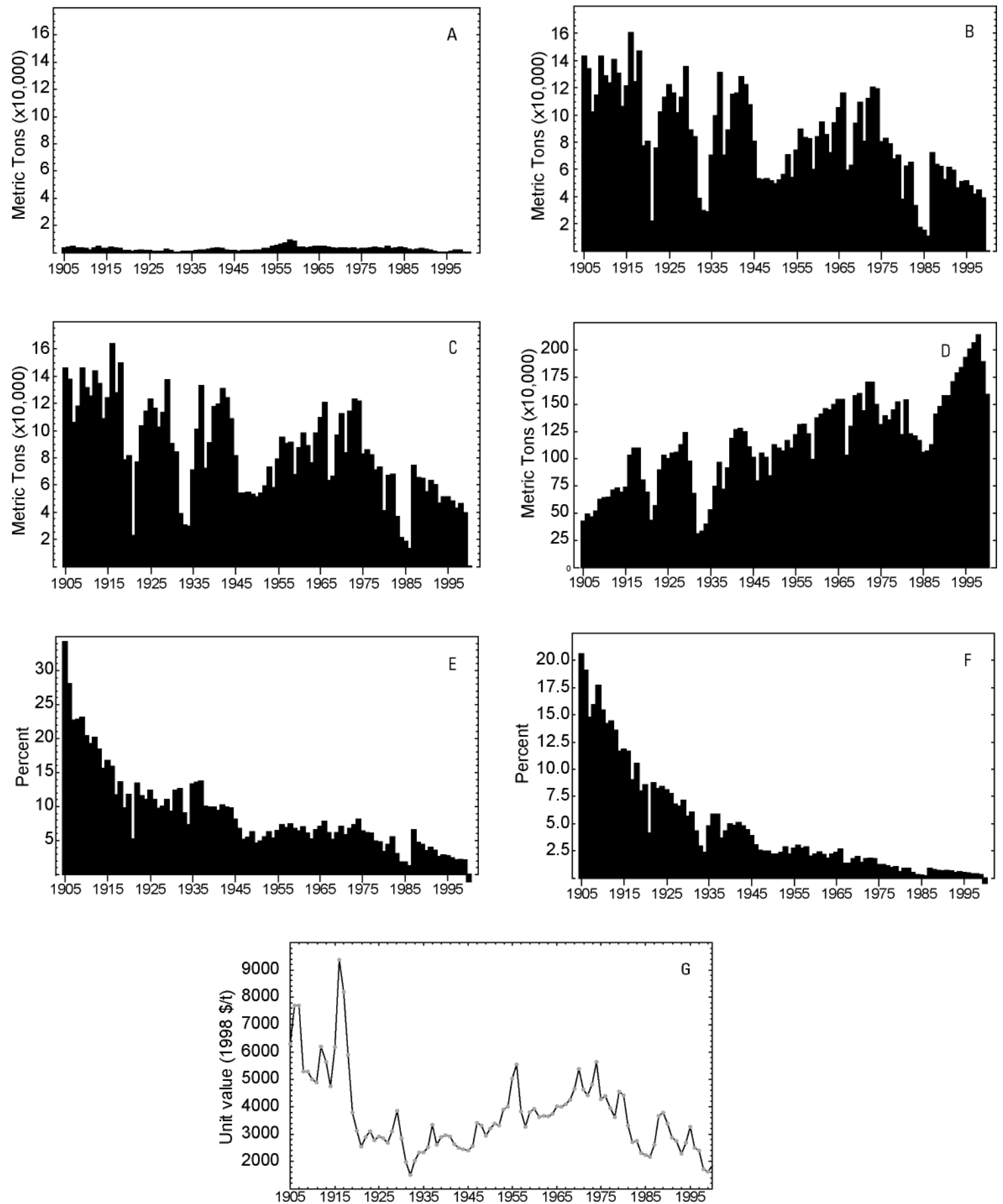

Figure 3. Copper production information from 1905-2001. A, annual production from Idaho. B, annual production from Montana. C, Idaho and Montana combined production. D, annual U.S. copper production. E, the proportion of regional copper production to U.S. primary production. $F$, the proportion of regional copper production to world mine production. $\mathrm{G}$, the historical value of copper in constant 1998 dollars. Bars extending below the $\mathrm{x}$-axis represent data withheld from publication. 


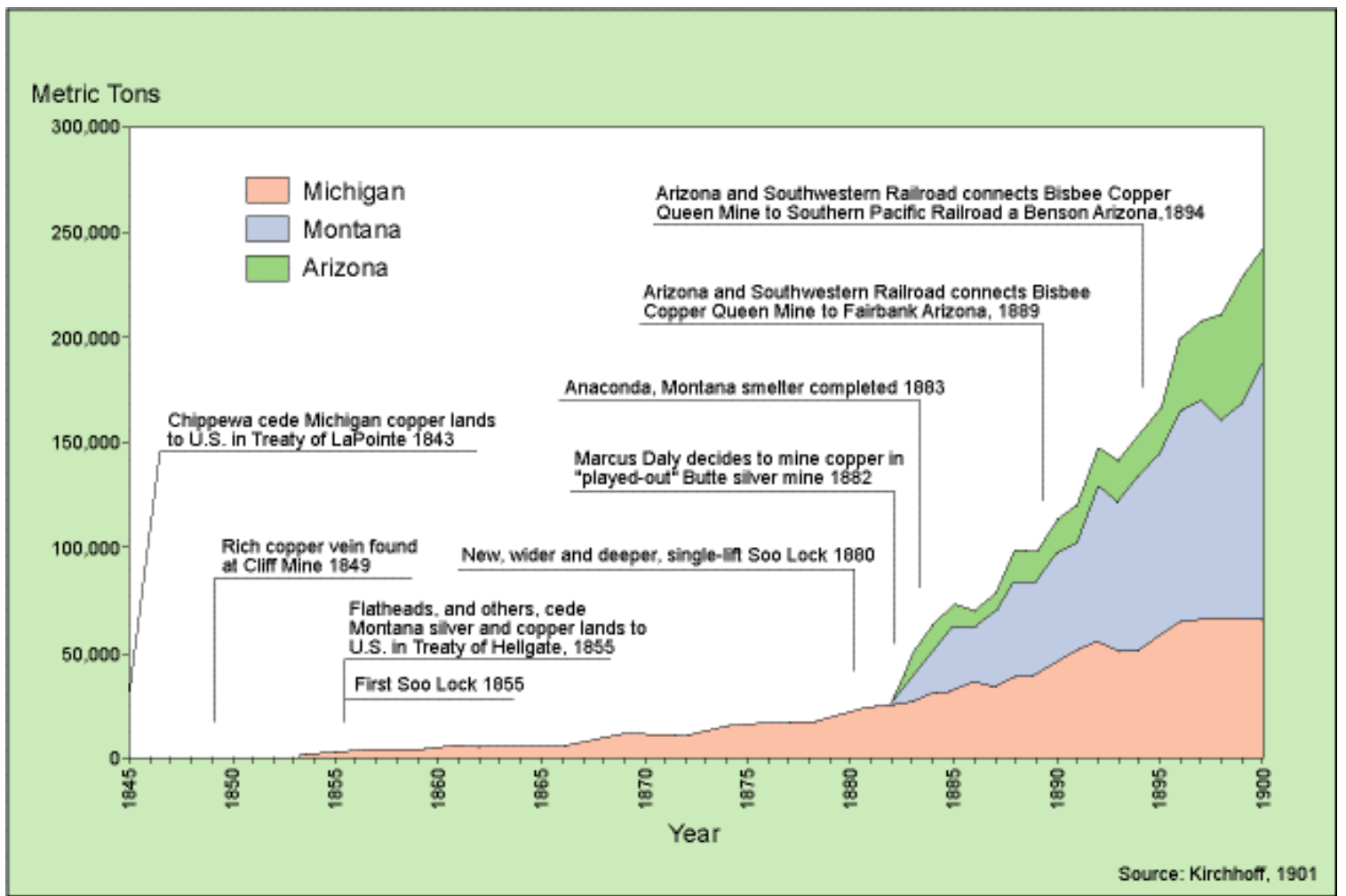

Figure 4. Nineteenth century copper production and associated events (from Goonan, 2002)

Table 8. Copper production from Idaho and Montana, the U.S., and the world, 1905-2000

[The table also summarizes the proportions of Idaho and Montana production relative to the US and the world as well as the price for copper in constant 1998 dollars. National copper production data represent total refined domestic and imported copper produced from ore, concentrate, or precipitate. Data for state, US, and world production are given in metric tons; proportions of US and world production by Idaho and Montana are given in percent; copper value is in US dollars. W, withheld]

\begin{tabular}{|c|c|c|c|c|c|c|c|c|}
\hline \multirow[b]{2}{*}{ Year } & \multicolumn{5}{|c|}{ Production } & \multicolumn{2}{|c|}{$\begin{array}{l}\text { Proportion of Idaho } \\
\text { and Montana } \\
\text { production to: }\end{array}$} & \multirow{2}{*}{$\begin{array}{r}\text { Coppe } \\
\text { value } \\
(1998 \$ / t) \\
\end{array}$} \\
\hline & Idaho & Montana & combined & US & World & US & World & \\
\hline 1905 & 3,321 & 142,771 & 146,092 & 426,000 & 713,000 & 34.29 & 20.49 & 6,300 \\
\hline 1906 & 3,891 & 133,676 & 137,567 & 489,000 & 724,000 & 28.13 & 19.00 & 7,700 \\
\hline 1907 & 4,403 & 101,726 & 106,129 & 468,000 & 721,000 & 22.68 & 14.72 & 7,700 \\
\hline 1908 & 3,291 & 114,536 & 117,827 & 516,000 & 744,000 & 22.83 & 15.84 & 5,300 \\
\hline 1909 & 3,219 & 142,820 & 146,039 & 631,000 & 828,000 & 23.14 & 17.64 & 5,300 \\
\hline 1910 & 3,120 & 128,404 & 131,524 & 645,000 & 858,000 & 20.39 & 15.33 & 5,000 \\
\hline 1911 & 2,048 & 123,295 & 125,343 & 650,000 & 890,000 & 19.28 & 14.08 & 4,900 \\
\hline 1912 & 3,398 & 140,498 & 143,896 & 711,000 & $1,000,000$ & 20.24 & 14.39 & 6,200 \\
\hline 1913 & 4,351 & 130,559 & 134,910 & 733,000 & 996,000 & 18.41 & 13.55 & 5,630 \\
\hline 1914 & 2,924 & 105,793 & 108,717 & 696,000 & 938,000 & 15.62 & 11.59 & 4,760 \\
\hline 1915 & 3,166 & 121,216 & 124,382 & 741,000 & $1,060,000$ & 16.79 & 11.73 & 6,180 \\
\hline 1916 & 3,846 & 160,088 & 163,934 & $1,030,000$ & $1,420,000$ & 15.92 & 11.54 & 9,360 \\
\hline 1917 & 3,551 & 124,496 & 128,047 & $1,100,000$ & $1,430,000$ & 11.64 & 8.95 & 8,190 \\
\hline
\end{tabular}




\begin{tabular}{|c|c|c|c|c|c|c|c|c|}
\hline 1918 & 2,964 & 146,592 & 149,556 & $1,100,000$ & $1,430,000$ & 13.60 & 10.46 & 5,890 \\
\hline 1919 & 1,416 & 77,104 & 78,520 & 803,000 & 994,000 & 9.78 & 7.90 & 3,780 \\
\hline 1920 & 1,151 & 80,314 & 81,465 & 692,000 & 959,000 & 11.77 & 8.49 & 3,140 \\
\hline 1921 & 766 & 21,818 & 22,583 & 431,000 & 558,000 & 5.24 & 4.05 & 2,540 \\
\hline 1922 & 1,489 & 75,186 & 76,675 & 569,000 & 884,000 & 13.48 & 8.67 & 2,900 \\
\hline 1923 & 1,805 & 101,663 & 103,468 & 898,000 & $1,270,000$ & 11.52 & 8.15 & 3,100 \\
\hline 1924 & 1,242 & 113,015 & 114,258 & $1,030,000$ & $1,360,000$ & 11.09 & 8.40 & 2,790 \\
\hline 1925 & 1,496 & 121,978 & 123,474 & $1,000,000$ & $1,530,000$ & 12.35 & 8.07 & 2,920 \\
\hline 1926 & 607 & 115,837 & 116,444 & $1,050,000$ & $1,510,000$ & 11.09 & 7.71 & 2,840 \\
\hline 1927 & 986 & 101,376 & 102,362 & $1,060,000$ & $1,520,000$ & 9.66 & 6.73 & 2,690 \\
\hline 1928 & 940 & 112,612 & 113,552 & $1,130,000$ & $1,730,000$ & 10.05 & 6.56 & 3,110 \\
\hline 1929 & 2,328 & 135,049 & 137,376 & $1,240,000$ & $1,950,000$ & 11.08 & 7.04 & 3,850 \\
\hline 1930 & 1,411 & 88,991 & 90,402 & 978,000 & $1,610,000$ & 9.24 & 5.62 & 2,860 \\
\hline 1931 & 519 & 83,714 & 84,234 & 681,000 & $1,400,000$ & 12.37 & 6.02 & 1,980 \\
\hline 1932 & 519 & 38,487 & 39,005 & 309,000 & 909,000 & 12.62 & 4.29 & 1,520 \\
\hline 1933 & 709 & 29,700 & 30,409 & 336,000 & $1,050,000$ & 9.05 & 2.90 & 2,020 \\
\hline 1934 & 695 & 28,697 & 29,392 & 404,000 & $1,280,000$ & 7.28 & 2.30 & 2,330 \\
\hline 1935 & 951 & 70,289 & 71,239 & 534,000 & $1,500,000$ & 13.34 & 4.75 & 2,330 \\
\hline 1936 & 1,340 & 99,378 & 100,718 & 746,000 & $1,720,000$ & 13.50 & 5.86 & 2,520 \\
\hline 1937 & 2,025 & 131,116 & 133,141 & 968,000 & $2,290,000$ & 13.75 & 5.81 & 3,350 \\
\hline 1938 & 1,941 & 70,048 & 71,988 & 719,000 & $1,990,000$ & 10.01 & 3.62 & 2,610 \\
\hline 1939 & 2,283 & 88,749 & 91,031 & 916,000 & $2,130,000$ & 9.94 & 4.27 & 2,900 \\
\hline 1940 & 3,038 & 114,662 & 117,700 & $1,190,000$ & $2,400,000$ & 9.89 & 4.90 & 2,960 \\
\hline 1941 & 3,285 & 116,154 & 119,439 & $1,270,000$ & $2,480,000$ & 9.40 & 4.82 & 2,930 \\
\hline 1942 & 3,112 & 128,091 & 131,203 & $1,280,000$ & $2,590,000$ & 10.25 & 5.07 & 2,650 \\
\hline 1943 & 2,108 & 122,041 & 124,149 & $1,250,000$ & $2,620,000$ & 9.93 & 4.74 & 2,500 \\
\hline 1944 & 1,531 & 107,222 & 108,753 & $1,110,000$ & $2,460,000$ & 9.80 & 4.42 & 2,450 \\
\hline 1945 & 1,404 & 80,293 & 81,697 & $1,010,000$ & $2,110,000$ & 8.09 & 3.87 & 2,410 \\
\hline 1946 & 942 & 53,054 & 53,996 & 797,000 & $1,780,000$ & 6.77 & 3.03 & 2,580 \\
\hline 1947 & 1,488 & 52,527 & 54,015 & $1,050,000$ & $2,130,000$ & 5.14 & 2.54 & 3,420 \\
\hline 1948 & 1,473 & 52,846 & 54,320 & $1,010,000$ & $2,210,000$ & 5.38 & 2.46 & 3,320 \\
\hline 1949 & 1,305 & 51,357 & 52,662 & 842,000 & $2,140,000$ & 6.25 & 2.46 & 2,940 \\
\hline 1950 & 1,911 & 49,422 & 51,334 & $1,130,000$ & $2,380,000$ & 4.54 & 2.16 & 3,210 \\
\hline 1951 & 1,960 & 52,079 & 54,038 & $1,100,000$ & $2,490,000$ & 4.91 & 2.17 & 3,400 \\
\hline 1952 & 2,915 & 56,199 & 59,114 & $1,070,000$ & $2,570,000$ & 5.52 & 2.30 & 3,310 \\
\hline 1953 & 2,845 & 70,414 & 73,259 & $1,170,000$ & $2,600,000$ & 6.26 & 2.82 & 3,910 \\
\hline 1954 & 4,380 & 53,841 & 58,221 & $1,100,000$ & $2,640,000$ & 5.29 & 2.21 & 4,000 \\
\hline 1955 & 5,097 & 73,975 & 79,072 & $1,220,000$ & $2,900,000$ & 6.48 & 2.73 & 5,040 \\
\hline 1956 & 6,038 & 89,292 & 95,330 & $1,310,000$ & $3,200,000$ & 7.28 & 2.98 & 5,540 \\
\hline 1957 & 7,178 & 83,020 & 90,197 & $1,320,000$ & $3,300,000$ & 6.83 & 2.73 & 3,840 \\
\hline 1958 & 8,932 & 82,268 & 91,200 & $1,230,000$ & $3,190,000$ & 7.41 & 2.86 & 3,280 \\
\hline 1959 & 7,904 & 59,794 & 67,699 & 996,000 & $3,430,000$ & 6.80 & 1.97 & 3,820 \\
\hline 1960 & 3,817 & 83,437 & 87,254 & $1,380,000$ & $3,940,000$ & 6.32 & 2.21 & 3,920 \\
\hline 1961 & 3,926 & 94,349 & 98,275 & $1,410,000$ & $4,090,000$ & 6.97 & 2.40 & 3,630 \\
\hline 1962 & 3,503 & 85,296 & 88,799 & $1,460,000$ & $4,220,000$ & 6.08 & 2.10 & 3,670 \\
\hline 1963 & 3,785 & 72,360 & 76,145 & $1,450,000$ & $4,290,000$ & 5.25 & 1.77 & 3,640 \\
\hline 1964 & 4,233 & 94,173 & 98,406 & $1,500,000$ & $4,450,000$ & 6.56 & 2.21 & 3,750 \\
\hline 1965 & 4,663 & 104,772 & 109,435 & $1,550,000$ & $4,660,000$ & 7.06 & 2.35 & 4,020 \\
\hline 1966 & 4,501 & 116,177 & 120,678 & $1,550,000$ & $4,580,000$ & 7.79 & 2.63 & 3,990 \\
\hline 1967 & 3,819 & 59,406 & 63,225 & $1,030,000$ & $4,630,000$ & 6.14 & 1.37 & 4,100 \\
\hline
\end{tabular}




\begin{tabular}{|c|c|c|c|c|c|c|c|c|}
\hline 1968 & 3,198 & 63,032 & 66,230 & $1,300,000$ & $5,010,000$ & 5.09 & 1.32 & 4,260 \\
\hline 1969 & 3,023 & 93,726 & 96,749 & $1,580,000$ & $5,520,000$ & 6.12 & 1.75 & 4,649 \\
\hline 1970 & 3,277 & 109,238 & 112,515 & $1,600,000$ & $5,900,000$ & 7.03 & 1.91 & 5,375 \\
\hline 1971 & 3,426 & 80,361 & 83,786 & $1,440,000$ & $5,940,000$ & 5.82 & 1.41 & 4,623 \\
\hline 1972 & 2,669 & 111,685 & 114,354 & $1,700,000$ & $6,540,000$ & 6.73 & 1.75 & 4,420 \\
\hline 1973 & 3,289 & 120,173 & 123,462 & $1,700,000$ & $6,920,000$ & 7.26 & 1.78 & 4,811 \\
\hline 1974 & 2,577 & 118,962 & 121,539 & $1,500,000$ & $7,100,000$ & 8.10 & 1.71 & 5,631 \\
\hline 1975 & 2,896 & 79,796 & 82,692 & $1,310,000$ & $6,740,000$ & 6.31 & 1.23 & 4,285 \\
\hline 1976 & 3,050 & 82,656 & 85,706 & $1,400,000$ & $7,260,000$ & 6.12 & 1.18 & 4,393 \\
\hline 1977 & 3,676 & 78,202 & 81,878 & $1,360,000$ & $7,420,000$ & 6.02 & 1.10 & 3,960 \\
\hline 1978 & 3,888 & 67,325 & 71,213 & $1,450,000$ & $7,280,000$ & 4.91 & 0.98 & 3,625 \\
\hline 1979 & 3,618 & 69,854 & 73,472 & $1,520,000$ & $7,350,000$ & 4.83 & 1.00 & 4,565 \\
\hline 1980 & 3,103 & 37,749 & 40,852 & $1,220,000$ & $7,200,000$ & 3.35 & 0.57 & 4,419 \\
\hline 1981 & 4,245 & 62,485 & 66,730 & $1,540,000$ & $7,690,000$ & 4.33 & 0.87 & 3,328 \\
\hline 1982 & 3,074 & 64,951 & 68,025 & $1,230,000$ & $7,580,000$ & 5.53 & 0.90 & 2,711 \\
\hline 1983 & 3,556 & 33,337 & 36,893 & $1,210,000$ & $7,610,000$ & 3.05 & 0.48 & 2,761 \\
\hline 1984 & 3,701 & 17,237 & 20,938 & $1,170,000$ & $7,810,000$ & 0.32 & 0.05 & 2,313 \\
\hline 1985 & 3,551 & 15,092 & 18,643 & $1,060,000$ & $7,990,000$ & 1.76 & 0.23 & 2,238 \\
\hline 1986 & 2,501 & 10,955 & 13,456 & $1,070,000$ & $7,940,000$ & 1.26 & 0.17 & 2,165 \\
\hline 1987 & 2,020 & 72,404 & 74,425 & $1,130,000$ & $8,240,000$ & 6.59 & 0.90 & 2,609 \\
\hline 1988 & 2,269 & 63,190 & 2,269 & $1,410,000$ & $8,720,000$ & 0.16 & 0.03 & 3,662 \\
\hline 1989 & 2,950 & 61,834 & 2,950 & $1,480,000$ & $9,040,000$ & 0.20 & 0.03 & 3,797 \\
\hline 1990 & 2,520 & 52,435 & 54,955 & $1,580,000$ & $9,200,000$ & 3.48 & 0.60 & 3,383 \\
\hline 1991 & 1,793 & 61,326 & 63,118 & $1,580,000$ & $9,330,000$ & 3.99 & 0.68 & 2,885 \\
\hline 1992 & 1,001 & 59,421 & 60,421 & $1,710,000$ & $9,470,000$ & 3.53 & 0.64 & 2,751 \\
\hline 1993 & 368 & 46,085 & 46,453 & $1,790,000$ & $9,490,000$ & 2.60 & 0.49 & 2,278 \\
\hline 1994 & 375 & 50,893 & 51,268 & $1,840,000$ & $9,500,000$ & 2.79 & 0.54 & 2,692 \\
\hline 1995 & 332 & 51,165 & 51,497 & $1,930,000$ & $10,000,000$ & 2.67 & 0.51 & 3,262 \\
\hline 1996 & 943 & 47,536 & 48,480 & $2,010,000$ & $11,000,000$ & 2.41 & 0.44 & 2,498 \\
\hline 1997 & 1,623 & 41,458 & 43,081 & $2,070,000$ & $11,400,000$ & 2.08 & 0.38 & 2,395 \\
\hline 1998 & 1,670 & 44,634 & 46,304 & $2,140,000$ & $12,100,000$ & 2.16 & 0.38 & 1,734 \\
\hline 1999 & 561 & 38,646 & 39,207 & $1,890,000$ & $12,700,000$ & 2.07 & 0.31 & 1,638 \\
\hline 2000 & 463 & W & 463 & $1,590,000$ & $13,200,000$ & W & W & 1,841 \\
\hline
\end{tabular}




\section{Fluorspar}

Regional fluorspar production is limited to the contribution from Montana as the fluorspar industry in Idaho was small and sporadic throughout the $20^{\text {th }}$ century. The Crystal Mountain open pit mine in the Bitterroot Valley of Montana was historically one of the world's largest fluorspar mines and was the primary source of fluorspar coming out of Montana in the 1950's. Montana was an abundant fluorspar producer from 1952 until 1977, shipping between 10,000 and 60,000 metric tons in the years from 19521961 before production reporting was withheld in subsequent years (fig. 5A). Prior to Montana's involvement in the fluorspar industry, production within the U.S. increased during the early thirties, owing to an expansion in the use of freon as a refrigerant (Kramer, undated). In the 1950's, Montana represented a large proportion of the national production, contributing nearly 20 percent in 1957 (fig. 5C). The large output from Montana during that decade similarly amounted to a large proportion of the world's production of fluorspar. Throughout the 1950's, between 1 and 3 percent of the world's fluorspar production originated in the state of Montana (fig. 5D). However, this positive trend reversed in the late 1960's, and Montana's production has declined ever since. The Montreal Protocol, introduced in 1987, called for the phasing out of fluorocarbons (Kramer, undated). This prompted a drastic decline in the price of fluorspar, which saw a $2 / 3$ devaluation of it's near high value of the early 1980 s (fig. $5 \mathrm{E}$ ). The price of the commodity never recovered, and the last U.S. fluorspar mine suspended operations in 1995 (Kramer, undated).

Table 9. Selected events affecting regional fluorspar production

\begin{tabular}{lll}
\hline Year & Event & Reference \\
\hline 1941 & Crystal Mountain deposit discovered & this study \\
\hline
\end{tabular}

Table 10. Selected events affecting U.S. fluorspar production

\begin{tabular}{lll}
\hline Year & Event & Reference \\
\hline $1914-18$ & WWI - high demand & Kramer, undated \\
1931 & Synthetic refrigerant freon first produced & \\
$1939-45$ & WWI - high demand & Kramer, undated \\
1987 & Montreal Protocol adopted. Phase out of chlorofluorocarbons & Kramer, undated \\
\hline
\end{tabular}



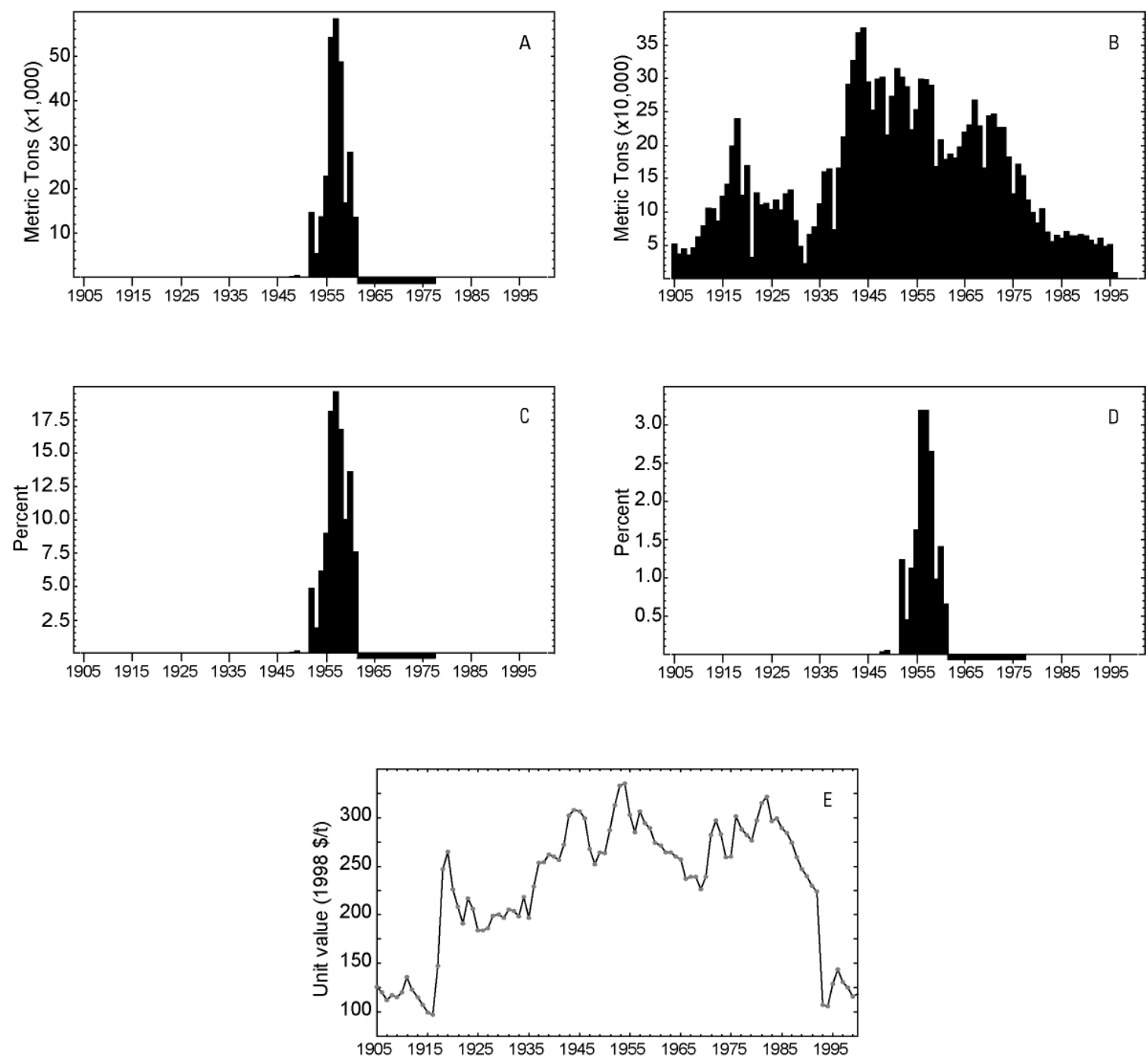

Figure 5. Fluorspar production information from 1905-2001. A, annual production from Montana. B, annual U.S. fluorspar production. C, the proportion of Montana fluorspar production to U.S. production. D, the proportion of Montana fluorspar production to world production. E, the historical value of fluorspar in constant 1998 dollars. Bars extending below the $x$-axis represent data withheld from publication.

Table 11. Fluorspar production from Idaho and Montana, the U.S., and the world, 1905-2000

[The table also summarizes the proportions of Idaho and Montana production relative to the US and the world as well as the price for fluorspar in constant 1998 dollars. Data for state, US, and world production are given in metric tons; proportions of US and world production by Idaho and Montana are given in percent; fluorspar value is in US dollars. W, withheld; leaders ( _ _ ), no production; n.d., no data]

\begin{tabular}{|c|c|c|c|c|c|c|}
\hline \multirow[b]{2}{*}{ Year } & \multicolumn{3}{|c|}{ Production } & \multicolumn{2}{|c|}{$\begin{array}{c}\text { Proportion of Montana production } \\
\text { to: }\end{array}$} & \multirow{2}{*}{$\begin{array}{r}\text { Fluorspar } \\
\text { value }(1998 \$ / t)\end{array}$} \\
\hline & Montana & US & World & US & World & \\
\hline 1905 & -- & 52,000 & n.d. & -- & -- & 126 \\
\hline 1906 & -- & 37,000 & n.d. & -- & -- & 120 \\
\hline 1907 & - - & 44,800 & n.d. & - - & - - & 112 \\
\hline
\end{tabular}




\begin{tabular}{|c|c|c|c|c|c|c|}
\hline 1908 & -- & 35,100 & n.d. & -- & -- & 117 \\
\hline 1909 & -- & 46,000 & n.d. & -- & -- & 115 \\
\hline 1910 & -- & 63,000 & n.d. & -- & -- & 120 \\
\hline 1911 & -- & 78,900 & n.d. & -- & -- & 136 \\
\hline 1912 & -- & 106,000 & n.d. & -- & -- & 123 \\
\hline 1913 & -- & 105,000 & 171,000 & -- & -- & 115 \\
\hline 1914 & -- & 86,300 & 121,000 & -- & -- & 107 \\
\hline 1915 & -- & 124,000 & 163,000 & -- & -- & 99 \\
\hline 1916 & -- & 141,000 & 201,000 & -- & -- & 97 \\
\hline 1917 & -- & 198,000 & 279,000 & -- & -- & 147 \\
\hline 1918 & -- & 239,000 & 313,000 & -- & -- & 247 \\
\hline 1919 & -- & 125,000 & 196,000 & -- & -- & 265 \\
\hline 1920 & -- & 169,000 & 264,000 & -- & -- & 226 \\
\hline 1921 & -- & 31,700 & 92,500 & -- & -- & 208 \\
\hline 1922 & -- & 128,000 & 208,000 & -- & -- & 191 \\
\hline 1923 & -- & 110,000 & 215,000 & -- & -- & 217 \\
\hline 1924 & -- & 113,000 & 255,000 & -- & -- & 206 \\
\hline 1925 & -- & 103,000 & 263,000 & -- & -- & 184 \\
\hline 1926 & -- & 117,000 & 310,000 & -- & -- & 184 \\
\hline 1927 & -- & 102,000 & 317,000 & -- & -- & 186 \\
\hline 1928 & -- & 127,000 & 345,000 & -- & -- & 199 \\
\hline 1929 & -- & 133,000 & 376,000 & -- & -- & 200 \\
\hline 1930 & -- & 86,900 & 291,000 & -- & -- & 197 \\
\hline 1931 & -- & 48,400 & 166,000 & -- & -- & 205 \\
\hline 1932 & -- & 22,900 & 128,000 & -- & -- & 204 \\
\hline 1933 & -- & 66,100 & 229,000 & -- & -- & 198 \\
\hline 1934 & -- & 77,700 & 286,000 & -- & -- & 218 \\
\hline 1935 & -- & 112,000 & 340,000 & -- & -- & 197 \\
\hline 1936 & -- & 160,000 & 455,000 & -- & -- & 229 \\
\hline 1937 & -- & 164,000 & 519,000 & -- & -- & 253 \\
\hline 1938 & -- & 72,900 & 456,000 & -- & -- & 254 \\
\hline 1939 & -- & 166,000 & 577,000 & -- & -- & 262 \\
\hline 1940 & -- & 212,000 & 616,000 & -- & -- & 260 \\
\hline 1941 & -- & 291,000 & 698,000 & -- & -- & 256 \\
\hline 1942 & -- & 327,000 & 883,000 & -- & -- & 272 \\
\hline 1943 & -- & 368,000 & $1,040,000$ & -- & -- & 302 \\
\hline 1944 & -- & 375,000 & $1,040,000$ & -- & -- & 308 \\
\hline 1945 & -- & 294,000 & 674,000 & -- & -- & 306 \\
\hline 1946 & -- & 252,000 & 524,000 & -- & -- & 299 \\
\hline 1947 & -- & 299,000 & 655,000 & -- & -- & 268 \\
\hline 1948 & 288 & 301,000 & 795,000 & 0.10 & 0.036 & 252 \\
\hline 1949 & 383 & 215,000 & 710,000 & 0.18 & 0.054 & 264 \\
\hline 1950 & 37 & 273,000 & 844,000 & 0.01 & 0.004 & 263 \\
\hline 1951 & -- & 315,000 & $1,030,000$ & -- & -- & 287 \\
\hline 1952 & 14,660 & 301,000 & $1,180,000$ & 4.87 & 1.242 & 313 \\
\hline 1953 & 5,382 & 288,000 & $1,210,000$ & 1.87 & 0.445 & 333 \\
\hline 1954 & 13,701 & 223,000 & $1,220,000$ & 6.14 & 1.123 & 335 \\
\hline 1955 & 22,882 & 254,000 & $1,410,000$ & 9.01 & 1.623 & 303 \\
\hline
\end{tabular}




\begin{tabular}{|c|c|c|c|c|c|c|}
\hline 1956 & 54,228 & 299,000 & $1,700,000$ & 18.14 & 3.190 & 285 \\
\hline 1957 & 58,368 & 298,000 & $1,830,000$ & 19.59 & 3.190 & 306 \\
\hline 1958 & 48,675 & 290,000 & $1,840,000$ & 16.78 & 2.645 & 294 \\
\hline 1959 & 16,821 & 168,000 & $1,720,000$ & 10.01 & 0.978 & 289 \\
\hline 1960 & 28,371 & 208,000 & $2,020,000$ & 13.64 & 1.404 & 274 \\
\hline 1961 & 13,522 & 179,000 & $2,060,000$ & 7.55 & 0.656 & 271 \\
\hline 1962 & W & 187,000 & $2,150,000$ & W & W & 264 \\
\hline 1963 & W & 181,000 & $2,150,000$ & W & W & 264 \\
\hline 1964 & W & 197,000 & $2,460,000$ & W & W & 260 \\
\hline 1965 & W & 219,000 & $2,770,000$ & W & W & 257 \\
\hline 1966 & W & 230,000 & $2,840,000$ & $\mathrm{~W}$ & W & 237 \\
\hline 1967 & W & 268,000 & $3,170,000$ & W & W & 239 \\
\hline 1968 & W & 229,000 & $3,640,000$ & W & W & 239 \\
\hline 1969 & W & 166,000 & $3,890,000$ & W & W & 226 \\
\hline 1970 & W & 244,000 & $4,190,000$ & W & W & 239 \\
\hline 1971 & W & 247,000 & $4,760,000$ & W & W & 282 \\
\hline 1972 & W & 227,000 & $4,530,000$ & W & W & 297 \\
\hline 1973 & W & 226,000 & $4,580,000$ & W & W & 283 \\
\hline 1974 & W & 182,000 & $4,860,000$ & W & W & 259 \\
\hline 1975 & W & 127,000 & $4,520,000$ & W & W & 260 \\
\hline 1976 & W & 171,000 & $4,320,000$ & W & W & 301 \\
\hline 1977 & W & 154,000 & $4,380,000$ & W & W & 288 \\
\hline 1978 & -- & 117,000 & $4,670,000$ & -- & -- & 282 \\
\hline 1979 & -- & 99,200 & $4,610,000$ & -- & -- & 276 \\
\hline 1980 & -- & 84,000 & $5,010,000$ & -- & -- & 297 \\
\hline 1981 & -- & 105,000 & $5,100,000$ & -- & -- & 315 \\
\hline 1982 & -- & 69,900 & $4,530,000$ & -- & -- & 321 \\
\hline 1983 & -- & 55,300 & $4,230,000$ & -- & -- & 296 \\
\hline 1984 & -- & 65,300 & $4,830,000$ & -- & -- & 299 \\
\hline 1985 & -- & 59,900 & $4,980,000$ & -- & -- & 289 \\
\hline 1986 & -- & 70,800 & $4,850,000$ & -- & -- & 284 \\
\hline 1987 & -- & 63,500 & $4,600,000$ & -- & -- & 274 \\
\hline 1988 & -- & 63,500 & $5,280,000$ & -- & -- & 259 \\
\hline 1989 & -- & 66,000 & $5,560,000$ & -- & -- & 247 \\
\hline 1990 & -- & 63,500 & $5,120,000$ & -- & -- & 240 \\
\hline 1991 & -- & 58,000 & $4,300,000$ & -- & -- & 230 \\
\hline 1992 & -- & 51,000 & $4,120,000$ & -- & -- & 224 \\
\hline 1993 & -- & 60,000 & $4,180,000$ & -- & -- & 107 \\
\hline 1994 & -- & 49,000 & $3,750,000$ & -- & -- & 106 \\
\hline 1995 & -- & 51,000 & $4,170,000$ & -- & -- & 129 \\
\hline 1996 & -- & 8,200 & $4,040,000$ & -- & -- & 144 \\
\hline 1997 & -- & -- & $4,170,000$ & -- & -- & 131 \\
\hline 1998 & -- & -- & $4,370,000$ & -- & -- & 125 \\
\hline 1999 & -- & -- & $4,420,000$ & -- & -- & 116 \\
\hline 2000 & -- & -- & $4,520,000$ & -- & -- & 119 \\
\hline
\end{tabular}




\section{Gold}

Gold mining in Idaho, Montana, and the U.S. over the last century show similar trends that are closely tied to price. The production of gold in both Idaho (fig. 6A) and Montana (fig. 6B) began increasing in the early 1930's until the demand for copper miners during World War II forced the closure of many non-essential gold mines (Kramer, undated). Though gold production in Montana and Idaho were similarly affected by the war, historic production levels from Montana have exceeded the production from Idaho by a factor of about three. By 1946, when restrictions on mining had been lifted, production in Idaho had decreased to less than 0.5 metric tons per year and in Montana to about one metric ton per year. This was much lower than pre-war production levels when Idaho and Montana combined production was more than 13 metric tons in 1940 (fig. 6C). In 1980, the price of gold ballooned to $\$ 850$ per ounce, which coincided with an increase in U.S. gold production, as well as an increase from both Idaho and Montana (Kramer, undated). These two peaks in production (around 1940 and again in the early eighties) each accounted for more than eight percent of the U.S. gold production (fig. 6E). The 1940 and 1980 peaks also figure prominently in world production (fig. 6F); however the largest proportion from Idaho and Montana came much earlier, in the beginning of the $20^{\text {th }}$ century. Historically, production of gold in Idaho, Montana, and the U.S., has varied with value, showing a close link between production and price (fig. 6G).

Table 12. Selected events affecting regional gold production

\begin{tabular}{lll}
\hline Year & Event & Reference \\
\hline 1931 & Gold Hill plant and mill destroyed in forest fire & this study \\
$1942-46$ & Boise Basin shut down during war & this study \\
$1979-98$ & Montana's Zortman-Landusky mine in operation & this study \\
1998 & Montana passes law banning use of cyanide in all future mining & this study \\
1999 & Beartrack mine in Idaho closes & this study \\
2000 & Continental mine in Montana suspends operations & this study \\
\hline
\end{tabular}

Table 13. Selected events affecting U.S. gold production

\begin{tabular}{|c|c|c|}
\hline Year & Event & Reference \\
\hline 1934 & Gold reserve act of 1934 enacted & Kramer, undated \\
\hline 1939-1945 & $\begin{array}{l}\text { WWII - Most gold mining curtailed when miners were needed for } \\
\text { critical minerals such as copper }\end{array}$ & Kramer, undated \\
\hline 1946 & Gold mining resumed & Kramer, undated \\
\hline 1980 & Gold price peaks at $\$ 850$ per ounce & Kramer, undated \\
\hline 1981 & $\begin{array}{l}\text { Development of low-grade Carlin-type and hot spring gold } \\
\text { deposits in Nevada, along with improved heap leach recovery } \\
\text { technology led to increased national production. }\end{array}$ & this study \\
\hline
\end{tabular}



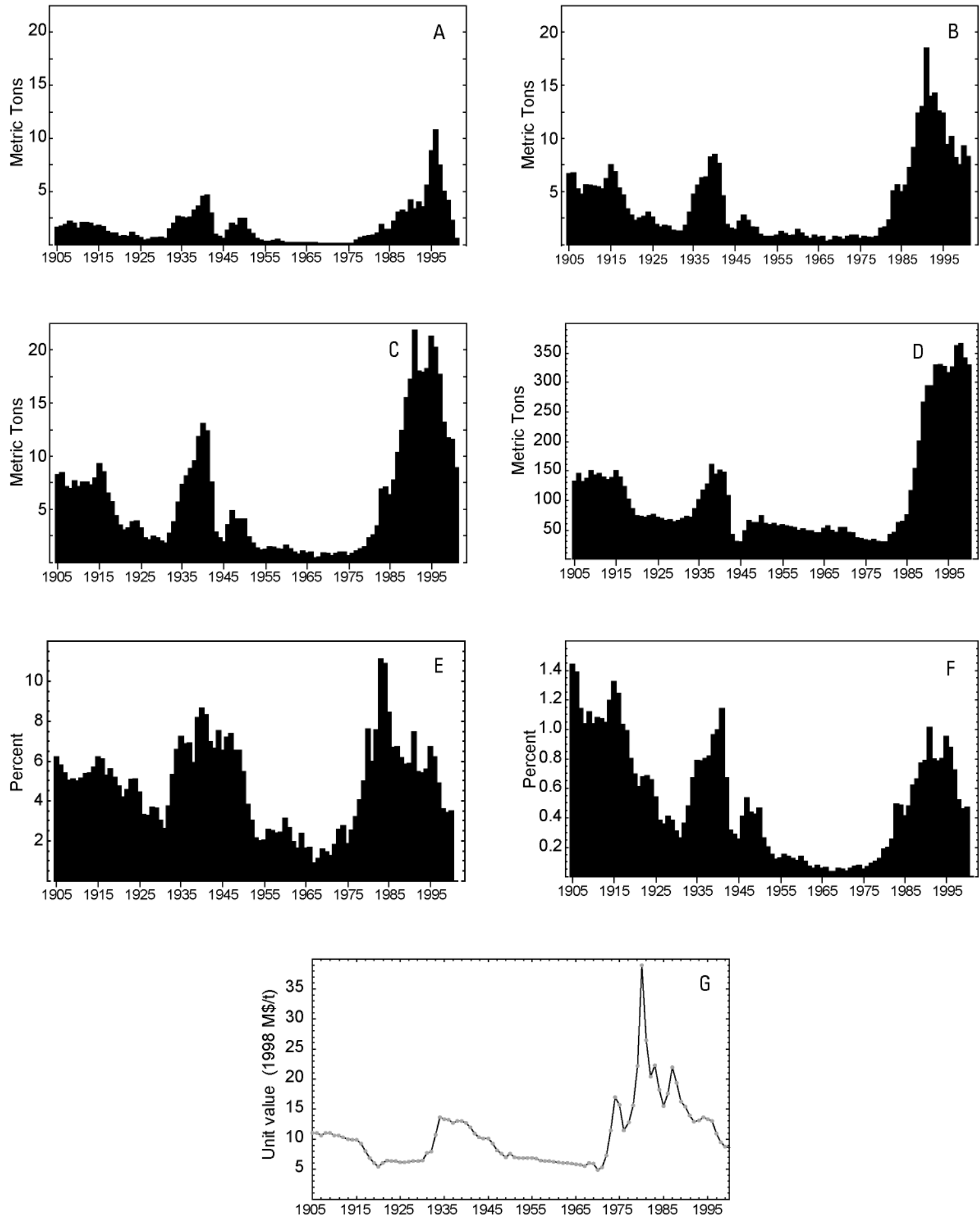

Figure 6. Gold production information from 1905-2001. A, annual production from Idaho. B, annual production from Montana. C, Idaho and Montana combined production. D, annual U.S. domestic mine production. E, the proportion of regional gold production to U.S. mine production. F, the proportion of regional gold production to world production. $G$, the historical value of gold in constant 1998 dollars. 
Table 14. Gold production from Idaho and Montana, the U.S., and the world, 1905-2000

[The table also summarizes the proportions of Idaho and Montana production relative to the US and the world as well as the price for gold in constant 1998 dollars. Data for state, US, and world production are given in metric tons; proportions of US and world production by Idaho and Montana are given in percent; gold value is in millions of US dollars per ton. W, withheld; n.d., no data]

\begin{tabular}{|c|c|c|c|c|c|c|c|c|}
\hline \multirow[b]{2}{*}{ Year } & \multicolumn{5}{|c|}{ Production } & \multicolumn{2}{|c|}{$\begin{array}{l}\text { Proportion of Idaho } \\
\text { and Montana } \\
\text { production to: }\end{array}$} & \multirow[b]{2}{*}{$\begin{array}{l}\text { Gold value } \\
\text { (1998 M\$/t) }\end{array}$} \\
\hline & Idaho & Montana & $\begin{array}{r}\text { Idaho and } \\
\text { Montana } \\
\text { combined } \\
\end{array}$ & US & World & US & World & \\
\hline 1905 & 1.62 & 6.65 & 8.27 & 133 & 575 & 6.22 & 1.44 & 11 \\
\hline 1906 & 1.73 & 6.72 & 8.45 & 146 & 608 & 5.79 & 1.39 & 11 \\
\hline 1907 & 1.89 & 5.22 & 7.11 & 132 & 623 & 5.39 & 1.14 & 10.6 \\
\hline 1908 & 2.17 & 4.75 & 6.93 & 138 & 668 & 5.02 & 1.04 & 11 \\
\hline 1909 & 2.02 & 5.64 & 7.67 & 150 & 687 & 5.11 & 1.12 & 11 \\
\hline 1910 & 1.56 & 5.60 & 7.16 & 143 & 689 & 5.00 & 1.04 & 10.6 \\
\hline 1911 & 2.04 & 5.49 & 7.53 & 146 & 699 & 5.16 & 1.08 & 10.6 \\
\hline 1912 & 2.08 & 5.45 & 7.53 & 140 & 705 & 5.38 & 1.07 & 10.3 \\
\hline 1913 & 2.02 & 5.26 & 7.28 & 135 & 694 & 5.39 & 1.05 & 10 \\
\hline 1914 & 1.73 & 6.20 & 7.93 & 139 & 663 & 5.70 & 1.20 & 9.9 \\
\hline 1915 & 1.78 & 7.53 & 9.30 & 150 & 704 & 6.20 & 1.32 & 9.89 \\
\hline 1916 & 1.68 & 6.85 & 8.53 & 140 & 685 & 6.09 & 1.24 & 9.3 \\
\hline 1917 & 1.21 & 5.29 & 6.50 & 123 & 631 & 5.29 & 1.03 & 8 \\
\hline 1918 & 1.06 & 4.67 & 5.73 & 102 & 578 & 5.62 & 0.99 & 6.87 \\
\hline 1919 & 1.07 & 3.35 & 4.43 & 85.6 & 550 & 5.17 & 0.81 & 6.04 \\
\hline 1920 & 0.73 & 2.79 & 3.52 & 74.1 & 507 & 4.74 & 0.69 & 5.37 \\
\hline 1921 & 0.82 & 2.23 & 3.05 & 72.9 & 498 & 4.18 & 0.61 & 6.03 \\
\hline 1922 & 0.75 & 2.49 & 3.25 & 71.3 & 481 & 4.55 & 0.68 & 6.49 \\
\hline 1923 & 1.15 & 2.65 & 3.80 & 74.8 & 554 & 5.08 & 0.69 & 6.35 \\
\hline 1924 & 0.84 & 3.04 & 3.88 & 76.0 & 592 & 5.11 & 0.66 & 6.34 \\
\hline 1925 & 0.65 & 2.55 & 3.20 & 71.8 & 591 & 4.46 & 0.54 & 6.18 \\
\hline 1926 & 0.43 & 1.88 & 2.31 & 69.4 & 602 & 3.32 & 0.38 & 6.11 \\
\hline 1927 & 0.48 & 1.67 & 2.14 & 65.5 & 597 & 3.27 & 0.36 & 6.24 \\
\hline 1928 & 0.65 & 1.81 & 2.46 & 66.8 & 603 & 3.69 & 0.41 & 6.33 \\
\hline 1929 & 0.63 & 1.70 & 2.33 & 64.0 & 609 & 3.65 & 0.38 & 6.31 \\
\hline 1930 & 0.67 & 1.35 & 2.02 & 66.5 & 648 & 3.04 & 0.31 & 6.47 \\
\hline 1931 & 0.57 & 1.25 & 1.82 & 69.2 & 695 & 2.63 & 0.26 & 7.74 \\
\hline 1932 & 1.46 & 1.26 & 2.72 & 72.5 & 754 & 3.75 & 0.36 & 7.94 \\
\hline 1933 & 2.01 & 1.80 & 3.81 & 71.7 & 793 & 5.31 & 0.48 & 10.7 \\
\hline 1934 & 2.64 & 3.03 & 5.67 & 86.4 & 841 & 6.56 & 0.67 & 13.6 \\
\hline 1935 & 2.61 & 4.70 & 7.31 & 101 & 924 & 7.23 & 0.79 & 13.3 \\
\hline 1936 & 2.50 & 5.61 & 8.10 & 118 & 1,030 & 6.87 & 0.79 & 13.2 \\
\hline 1937 & 2.55 & 6.29 & 8.84 & 128 & 1,100 & 6.90 & 0.80 & 12.7 \\
\hline 1938 & 3.22 & 6.32 & 9.54 & 161 & 1,170 & 5.93 & 0.82 & 13 \\
\hline 1939 & 3.63 & 8.22 & 11.85 & 145 & 1,230 & 8.17 & 0.96 & 13 \\
\hline 1940 & 4.56 & 8.48 & 13.03 & 151 & 1,310 & 8.63 & 1.00 & 12.7 \\
\hline 1941 & 4.66 & 7.67 & 12.33 & 148 & 1,080 & 8.33 & 1.14 & 12 \\
\hline 1942 & 2.96 & 4.57 & 7.52 & 108 & 1,120 & 6.97 & 0.67 & 10.9 \\
\hline 1943 & 0.96 & 1.85 & 2.81 & 42.4 & 896 & 6.63 & 0.31 & 10.3 \\
\hline 1944 & 0.78 & 1.56 & 2.33 & 31.1 & 813 & 7.50 & 0.29 & 10.1 \\
\hline
\end{tabular}




\begin{tabular}{|c|c|c|c|c|c|c|c|c|}
\hline 1945 & 0.55 & 1.39 & 1.94 & 29.7 & 762 & 6.53 & 0.25 & 10.1 \\
\hline 1946 & 1.34 & 2.19 & 3.53 & 49.0 & 860 & 7.20 & 0.41 & 9.32 \\
\hline 1947 & 2.02 & 2.80 & 4.82 & 65.6 & 900 & 7.35 & 0.54 & 8.15 \\
\hline 1948 & 1.82 & 2.27 & 4.09 & 62.7 & 932 & 6.53 & 0.44 & 7.56 \\
\hline 1949 & 2.42 & 1.64 & 4.06 & 62.0 & 964 & 6.55 & 0.42 & 6.97 \\
\hline 1950 & 2.48 & 1.61 & 4.09 & 74.5 & 879 & 5.49 & 0.47 & 7.56 \\
\hline 1951 & 1.40 & 0.95 & 2.35 & 61.6 & 883 & 3.82 & 0.27 & 7.01 \\
\hline 1952 & 1.03 & 0.75 & 1.78 & 58.9 & 868 & 3.02 & 0.20 & 6.83 \\
\hline 1953 & 0.55 & 0.77 & 1.32 & 60.9 & 864 & 2.17 & 0.15 & 6.83 \\
\hline 1954 & 0.41 & 0.74 & 1.15 & 57.1 & 965 & 2.01 & 0.12 & 6.83 \\
\hline 1955 & 0.33 & 0.87 & 1.20 & 58.5 & 947 & 2.06 & 0.13 & 6.86 \\
\hline 1956 & 0.29 & 1.19 & 1.47 & 56.8 & 978 & 2.59 & 0.15 & 6.75 \\
\hline 1957 & 0.38 & 1.02 & 1.40 & 55.8 & 1,020 & 2.51 & 0.14 & 6.51 \\
\hline 1958 & 0.49 & 0.81 & 1.30 & 54.1 & 1,050 & 2.41 & 0.12 & 6.36 \\
\hline 1959 & 0.33 & 0.89 & 1.21 & 49.9 & 1,130 & 2.43 & 0.11 & 6.31 \\
\hline 1960 & 0.19 & 1.43 & 1.62 & 51.8 & 1,190 & 3.13 & 0.14 & 6.24 \\
\hline 1961 & 0.18 & 1.10 & 1.28 & 48.2 & 1,230 & 2.65 & 0.10 & 6.16 \\
\hline 1962 & 0.18 & 0.76 & 0.94 & 48.0 & 1,290 & 1.96 & 0.07 & 6.09 \\
\hline 1963 & 0.17 & 0.58 & 0.75 & 45.2 & 1,340 & 1.65 & 0.06 & 6.01 \\
\hline 1964 & 0.18 & 0.91 & 1.08 & 45.3 & 1,390 & 2.39 & 0.08 & 5.93 \\
\hline 1965 & 0.16 & 0.71 & 0.87 & 53.0 & 1,440 & 1.63 & 0.06 & 5.84 \\
\hline 1966 & 0.16 & 0.78 & 0.94 & 56.1 & 1,450 & 1.67 & 0.06 & 5.68 \\
\hline 1967 & 0.15 & 0.30 & 0.45 & 49.3 & 1,420 & 0.92 & 0.03 & 5.53 \\
\hline 1968 & 0.10 & 0.42 & 0.52 & 46.0 & 1,440 & 1.12 & 0.04 & 6.04 \\
\hline 1969 & 0.11 & 0.75 & 0.86 & 53.9 & 1,450 & 1.59 & 0.06 & 5.94 \\
\hline 1970 & 0.10 & 0.70 & 0.80 & 54.2 & 1,480 & 1.47 & 0.05 & 4.92 \\
\hline 1971 & 0.11 & 0.49 & 0.60 & 46.5 & 1,450 & 1.28 & 0.04 & 5.34 \\
\hline 1972 & 0.09 & 0.74 & 0.83 & 45.1 & 1,390 & 1.84 & 0.06 & 7.34 \\
\hline 1973 & 0.08 & 0.86 & 0.95 & 36.6 & 1,350 & 2.59 & 0.07 & 11.5 \\
\hline 1974 & 0.09 & 0.88 & 0.97 & 35.1 & 1,250 & 2.76 & 0.08 & 17 \\
\hline 1975 & 0.08 & 0.54 & 0.62 & 32.7 & 1,200 & 1.88 & 0.05 & 15.7 \\
\hline 1976 & 0.09 & 0.75 & 0.83 & 32.6 & 1,210 & 2.56 & 0.07 & 11.5 \\
\hline 1977 & 0.40 & 0.70 & 1.10 & 34.2 & 1,210 & 3.21 & 0.09 & 12.8 \\
\hline 1978 & 0.64 & 0.62 & 1.26 & 31.1 & 1,210 & 4.05 & 0.10 & 15.6 \\
\hline 1979 & 0.75 & 0.75 & 1.50 & 30.0 & 1,210 & 5.00 & 0.12 & 22.2 \\
\hline 1980 & 0.79 & 1.50 & 2.29 & 30.2 & 1,220 & 7.60 & 0.19 & 39 \\
\hline 1981 & 0.87 & 1.69 & 2.56 & 42.9 & 1,280 & 5.97 & 0.20 & 26.5 \\
\hline 1982 & 1.10 & 2.34 & 3.44 & 45.6 & 1,340 & 7.54 & 0.26 & 20.4 \\
\hline 1983 & 1.89 & 5.02 & 6.91 & 62.3 & 1,400 & 11.09 & 0.49 & 22.3 \\
\hline 1984 & 1.41 & 5.64 & 7.05 & 64.9 & 1,460 & 10.86 & 0.48 & 18.2 \\
\hline 1985 & 1.38 & 4.98 & 6.36 & 75.5 & 1,530 & 8.43 & 0.42 & 15.5 \\
\hline 1986 & 2.19 & 5.56 & 7.75 & 116 & 1,610 & 6.68 & 0.48 & 17.6 \\
\hline 1987 & 3.04 & 7.29 & 10.33 & 154 & 1,660 & 6.71 & 0.62 & 22 \\
\hline 1988 & 3.22 & 9.17 & 12.39 & 201 & 1,870 & 6.17 & 0.66 & 19.4 \\
\hline 1989 & 3.06 & 12.43 & 15.49 & 266 & 2,010 & 5.82 & 0.77 & 16.2 \\
\hline 1990 & 4.22 & 13.01 & 17.23 & 294 & 2,180 & 5.86 & 0.79 & 15.4 \\
\hline 1991 & 3.35 & 18.51 & 21.86 & 294 & 2,160 & 7.44 & 1.01 & 14 \\
\hline 1992 & 4.04 & 13.99 & 18.03 & 330 & 2,260 & 5.46 & 0.80 & 12.9 \\
\hline 1993 & 3.53 & 14.33 & 17.85 & 331 & 2,280 & 5.39 & 0.78 & 13.1 \\
\hline 1994 & 5.60 & 12.60 & 18.20 & 327 & 2,260 & 5.57 & 0.81 & 13.6 \\
\hline
\end{tabular}




\begin{tabular}{rrrrrrrrr}
1995 & 8.85 & 12.40 & 21.25 & 317 & 2,230 & 6.70 & 0.95 & 13.3 \\
1996 & 10.80 & 9.44 & 20.24 & 326 & 2,300 & 6.21 & 0.88 & 13 \\
1997 & 7.49 & 10.20 & 17.69 & 362 & 2,450 & 4.89 & 0.72 & 10.9 \\
1998 & 4.99 & 8.20 & 13.19 & 366 & 2,520 & 3.60 & 0.52 & 9.49 \\
1999 & 4.17 & 7.54 & 11.71 & 341 & 2,540 & 3.43 & 0.46 & 8.78 \\
2000 & 2.26 & 9.31 & 11.57 & 330 & 2,450 & 3.51 & 0.47 & 8.59 \\
2001 & 0.59 & 8.28 & 8.87 & n.d. & n.d. & W & n.d. & n.d. \\
\hline
\end{tabular}




\section{Iron}

Iron ore production from Montana was limited and often sporadic and there is no recorded production of iron from Idaho. From 1905 to 2001, there were two periods of activity when iron ore mining operations increased in Montana (fig. 7A). Iron ore production began in 1914 and had increased to over 26,000 metric tons by 1921. However, a long period of inactivity occurred between 1929 and 1952. The most prolific year for Montana's iron ore industry was 1960, when nearly 56,000 metric tons of iron ore were shipped. Iron production records in Montana were mostly withheld after 1976. Like Montana, U.S. iron production levels also varied widely by year, but national trends do not show the sharp production peaks that are seen in the trends from Montana (fig. 7B). The minor role of Montana in U.S. iron production is shown in figure $7 \mathrm{C}$ where Montana accounts for less than 0.1 percent in any given year. Because of the very small numbers associated with Montana iron production as a percentage of the world's production, a chart was not produced for this data.
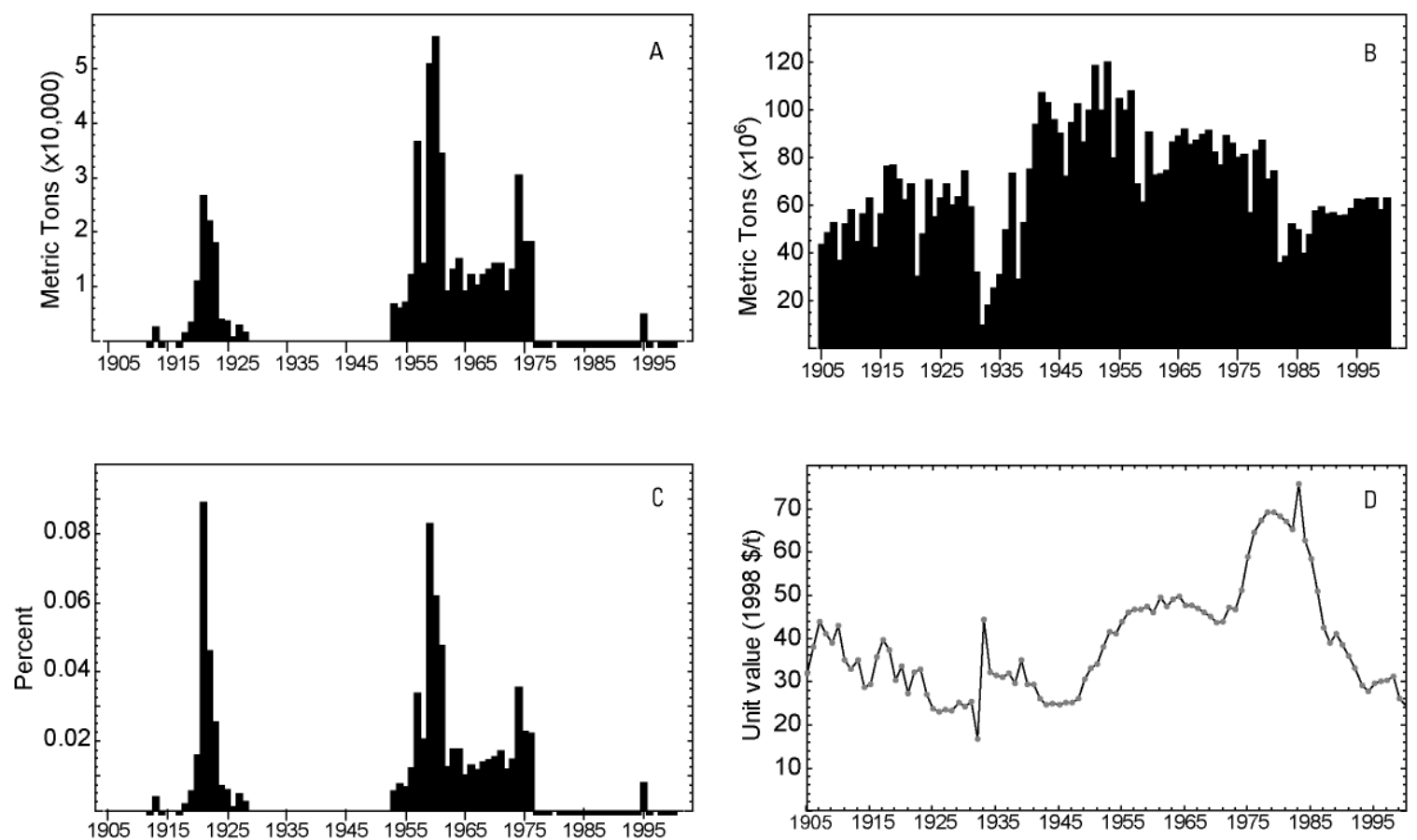

Figure 7. Iron production information from 1905-2001. A, annual production from Montana. B, annual U.S. production. C, the proportion of regional iron production to U.S. production. D, the historical value of iron in constant 1998 dollars. Bars extending below the $x$-axis represent data withheld from publication.

Table 15. Iron production from Montana, the U.S., and the world, 1905-2000

[The table also summarizes the proportions of Montana production relative to the US and the world as well as the price for iron in constant 1998 dollars. Data for state, US, and world production are given in metric tons; proportions of US and world production by Montana are given in percent; iron value is in US dollars. W, withheld; leaders ( $\_$_ $)$, no production; n.d., no data]

\begin{tabular}{|c|c|c|c|c|c|c|}
\hline \multirow[b]{2}{*}{ Year } & \multicolumn{3}{|c|}{ Production } & \multicolumn{2}{|c|}{$\begin{array}{l}\text { Proportion of Montana } \\
\text { production to: }\end{array}$} & \multirow[b]{2}{*}{$\begin{array}{r}\text { Iron value } \\
(1998 \$ / t)\end{array}$} \\
\hline & Montana & US & World & US & World & \\
\hline 1905 & - - & $43,209,000$ & $115,567,600$ & - - & -- & 32.00 \\
\hline
\end{tabular}




\begin{tabular}{|c|c|c|c|c|c|c|}
\hline 1906 & -- & $48,516,000$ & -- & -- & -- & 38.00 \\
\hline 1907 & -- & $52,551,000$ & -- & -- & -- & 44.00 \\
\hline 1908 & -- & $36,561,000$ & -- & -- & -- & 41.00 \\
\hline 1909 & -- & $51,976,000$ & -- & -- & -- & 39.00 \\
\hline 1910 & -- & $57,803,000$ & $160,074,200$ & -- & -- & 43.00 \\
\hline 1911 & -- & $44,581,000$ & $130,974,700$ & -- & -- & 35.00 \\
\hline 1912 & W & $56,056,000$ & $149,053,100$ & $\mathrm{~W}$ & W & 33.00 \\
\hline 1913 & 2,515 & $62,975,000$ & $180,419,900$ & 0.004 & 0.001 & 34.90 \\
\hline 1914 & $\mathrm{~W}$ & $42,105,000$ & $115,739,600$ & W & $\mathrm{W}$ & 28.60 \\
\hline 1915 & -- & $56,418,000$ & $139,304,500$ & -- & -- & 29.40 \\
\hline 1916 & -- & $76,374,000$ & $141,959,100$ & -- & -- & 35.80 \\
\hline 1917 & W & $76,497,000$ & $126,849,200$ & W & W & 39.70 \\
\hline 1918 & 1,438 & $70,776,000$ & $110,411,180$ & 0.002 & 0.001 & 37.40 \\
\hline 1919 & 3,493 & $61,944,000$ & $124,085,500$ & 0.006 & 0.003 & 30.20 \\
\hline 1920 & 10,976 & $68,690,000$ & $124,085,500$ & 0.016 & 0.009 & 33.70 \\
\hline 1921 & 26,594 & $29,964,000$ & $104,230,200$ & 0.089 & 0.026 & 27.30 \\
\hline 1922 & 22,075 & $47,885,000$ & $132,067,900$ & 0.046 & 0.017 & 32.20 \\
\hline 1923 & 18,036 & $70,465,000$ & $129,711,400$ & 0.026 & 0.014 & 32.80 \\
\hline 1924 & 3,976 & $55,138,000$ & $151,267,000$ & 0.007 & 0.003 & 27.00 \\
\hline 1925 & 3,731 & $62,902,000$ & $154,903,000$ & 0.006 & 0.002 & 23.80 \\
\hline 1926 & 736 & $68,708,000$ & $171,300,000$ & 0.001 & 0.000 & 23.10 \\
\hline 1927 & 2,883 & $60,158,000$ & $171,380,000$ & 0.005 & 0.002 & 23.40 \\
\hline 1928 & 1,666 & $63,195,000$ & $199,500,000$ & 0.003 & 0.001 & 23.30 \\
\hline 1929 & -- & $74,200,000$ & $178,400,000$ & -- & -- & 25.20 \\
\hline 1930 & -- & $59,346,000$ & $118,780,000$ & -- & -- & 24.20 \\
\hline 1931 & -- & $31,631,000$ & $118,780,000$ & -- & -- & 25.30 \\
\hline 1932 & -- & $9,639,200$ & $76,200,000$ & -- & -- & 16.80 \\
\hline 1933 & -- & $17,835,000$ & $91,200,000$ & -- & -- & 44.30 \\
\hline 1934 & -- & $24,982,000$ & $120,100,000$ & -- & -- & 32.20 \\
\hline 1935 & -- & $31,030,000$ & $140,900,000$ & - - & -- & 31.60 \\
\hline 1936 & -- & $49,572,000$ & $170,000,000$ & -- & -- & 31.10 \\
\hline 1937 & -- & $73,251,000$ & $211,000,000$ & -- & -- & 32.00 \\
\hline 1938 & -- & $28,904,000$ & $162,000,000$ & -- & -- & 29.60 \\
\hline 1939 & -- & $52,562,000$ & $200,000,000$ & -- & -- & 35.10 \\
\hline 1940 & -- & $74,879,000$ & $203,600,000$ & -- & -- & 29.30 \\
\hline 1941 & -- & $93,893,000$ & $219,540,000$ & -- & -- & 29.30 \\
\hline 1942 & -- & $107,220,000$ & $235,000,000$ & -- & -- & 26.10 \\
\hline 1943 & -- & $102,870,000$ & $231,000,000$ & - - & -- & 24.70 \\
\hline 1944 & -- & $95,628,000$ & $203,000,000$ & -- & -- & 24.90 \\
\hline 1945 & -- & $89,795,000$ & $162,000,000$ & -- & -- & 24.70 \\
\hline 1946 & -- & $71,980,000$ & $154,000,000$ & -- & -- & 25.10 \\
\hline 1947 & -- & $94,586,000$ & $187,000,000$ & -- & -- & 25.10 \\
\hline 1948 & -- & $102,620,000$ & $219,000,000$ & -- & -- & 26.20 \\
\hline 1949 & -- & $86,301,000$ & $223,000,000$ & -- & -- & 30.50 \\
\hline 1950 & -- & $99,619,000$ & $294,272,000$ & -- & -- & 33.20 \\
\hline 1951 & -- & $118,370,000$ & $297,295,300$ & -- & -- & 34.00 \\
\hline 1952 & -- & $99,490,000$ & $338,103,800$ & -- & -- & 38.10 \\
\hline 1953 & 6,817 & $119,890,000$ & $305,762,000$ & 0.006 & 0.002 & 41.50 \\
\hline
\end{tabular}




\begin{tabular}{|c|c|c|c|c|c|c|}
\hline 1954 & 6,096 & $79,383,000$ & $305,762,000$ & 0.008 & 0.002 & 41.00 \\
\hline 1955 & 7,112 & $104,660,000$ & $369,248,700$ & 0.007 & 0.002 & 44.00 \\
\hline 1956 & 12,193 & $99,448,000$ & $394,511,700$ & 0.012 & 0.003 & 46.00 \\
\hline 1957 & 36,578 & $107,850,000$ & $433,523,800$ & 0.034 & 0.008 & 46.80 \\
\hline 1958 & 14,225 & $68,796,000$ & $398,740,000$ & 0.021 & 0.004 & 46.70 \\
\hline 1959 & 50,802 & $61,243,000$ & $432,182,000$ & 0.083 & 0.012 & 47.40 \\
\hline 1960 & 55,883 & $90,209,000$ & $513,926,000$ & 0.062 & 0.011 & 45.90 \\
\hline 1961 & 34,546 & $72,474,000$ & $494,604,000$ & 0.048 & 0.007 & 49.60 \\
\hline 1962 & 9,144 & $72,982,000$ & $499,710,000$ & 0.013 & 0.002 & 47.40 \\
\hline 1963 & 13,209 & $74,780,000$ & $515,135,000$ & 0.018 & 0.003 & 49.00 \\
\hline 1964 & 15,241 & $86,198,000$ & $573,449,000$ & 0.018 & 0.003 & 49.80 \\
\hline 1965 & 9,144 & $88,842,000$ & $611,187,000$ & 0.010 & 0.001 & 47.70 \\
\hline 1966 & 12,193 & $91,594,000$ & $625,799,000$ & 0.013 & 0.002 & 47.70 \\
\hline 1967 & 10,160 & $85,530,000$ & $612,820,000$ & 0.012 & 0.002 & 47.00 \\
\hline 1968 & 12,193 & $87,243,000$ & $668,142,000$ & 0.014 & 0.002 & 45.90 \\
\hline 1969 & 13,209 & $89,746,000$ & $701,495,000$ & 0.015 & 0.002 & 45.13 \\
\hline 1970 & 14,225 & $91,201,000$ & $757,013,000$ & 0.016 & 0.002 & 43.62 \\
\hline 1971 & 14,225 & $82,058,000$ & $774,677,000$ & 0.017 & 0.002 & 43.96 \\
\hline 1972 & 9,144 & $76,645,000$ & $765,465,000$ & 0.012 & 0.001 & 47.12 \\
\hline 1973 & 13,209 & $89,076,000$ & $832,343,000$ & 0.015 & 0.002 & 46.77 \\
\hline 1974 & 30,481 & $85,709,000$ & $881,244,000$ & 0.036 & 0.003 & 51.24 \\
\hline 1975 & 18,289 & $80,132,000$ & $901,551,000$ & 0.023 & 0.002 & 58.89 \\
\hline 1976 & 18,289 & $81,277,000$ & $855,098,000$ & 0.023 & 0.002 & 64.60 \\
\hline 1977 & W & $56,645,000$ & $827,816,000$ & W & W & 67.26 \\
\hline 1978 & W & $82,892,000$ & $833,274,000$ & W & W & 69.32 \\
\hline 1979 & W & $87,092,000$ & $888,789,000$ & W & W & 69.16 \\
\hline 1980 & -- & $70,730,000$ & $877,152,000$ & -- & -- & 68.22 \\
\hline 1981 & $\mathrm{~W}$ & $74,348,000$ & $844,606,000$ & W & W & 67.14 \\
\hline 1982 & W & $36,002,000$ & $768,566,000$ & W & W & 65.33 \\
\hline 1983 & $\mathrm{~W}$ & $38,165,000$ & $728,247,000$ & W & W & 75.78 \\
\hline 1984 & $\mathrm{~W}$ & $52,092,000$ & $860,556,000$ & W & W & 62.66 \\
\hline 1985 & W & $49,533,000$ & $863,650,000$ & W & W & 58.47 \\
\hline 1986 & W & $39,486,000$ & $902,737,000$ & W & W & 50.88 \\
\hline 1987 & W & $47,648,000$ & $967,218,000$ & W & W & 42.52 \\
\hline 1988 & W & $57,515,000$ & $1,013,383,000$ & W & W & 39.04 \\
\hline 1989 & W & $59,032,000$ & $983,000,000$ & W & W & 41.18 \\
\hline 1990 & W & $56,400,000$ & $955,618,000$ & W & W & 38.54 \\
\hline 1991 & W & $56,761,000$ & $924,887,000$ & W & W & 36.03 \\
\hline 1992 & W & $55,593,000$ & $953,316,000$ & W & W & 33.21 \\
\hline 1993 & W & $55,676,000$ & $991,858,000$ & W & W & 29.11 \\
\hline 1994 & W & $58,454,000$ & $991,858,000$ & W & W & 27.67 \\
\hline 1995 & 5,000 & $62,581,000$ & $1,034,539,000$ & 0.008 & 0.000 & 29.66 \\
\hline 1996 & W & $62,083,000$ & $1,018,436,000$ & W & W & 30.03 \\
\hline 1997 & -- & $62,971,000$ & $1,068,727,000$ & -- & -- & 30.39 \\
\hline 1998 & W & $62,931,000$ & $1,050,688,000$ & W & W & 31.16 \\
\hline 1999 & W & $57,749,000$ & $1,019,051,000$ & W & W & 26.19 \\
\hline 2000 & W & $63,089,000$ & $1,061,148,000$ & W & W & 24.44 \\
\hline 2001 & -- & n.d. & n.d. & n.d. & n.d. & n.d. \\
\hline
\end{tabular}




\section{Lead}

Historically, Idaho has been a prominent lead producing state, specifically from the Coeur d' Alene mining district. The most abundant year for Idaho production was 1917 when nearly 180,000 metric tons of lead were refined (fig. 8A). Production rates overall have steadily declined in Idaho after the peak achieved early in the $20^{\text {th }}$ century. The average production of lead from Montana has been much lower than the average from Idaho, with Montana showing recorded production of less than 25,000 metric tons per year (fig. 8B). Together, Idaho and Montana have experienced somewhat cyclical waxing and waning in annual production; however, the overall trend has decreased over the last century (fig. 8C). A reduction in the use of lead in paint caused lower demand in the early 1950's; however, the decline in the national lead production (fig. 8D) was largely aided in 1973 when governmental regulations began phasing out lead in gasoline and all but banned it's use in indoor paint (Kramer, undated). In the early part of the $20^{\text {th }}$ century, Idaho and Montana combined to provide nearly 35 percent of the nation's lead supply (fig. 8E). After recognition of the toxic effects of lead, laws were enacted to limit commercial use, and production began to decline. By 2001, the proportion of the U.S. production from Idaho and Montana had dropped to less than 3 percent. Similarly, almost 16 percent of the world's lead production once originated in Idaho and Montana, though that has dropped to less than 0.5 percent in recent years (fig. 8F). An upturn in the price of lead in the 1990's (fig. 8G) was attributed to an increase in the demand for lead acid batteries; however, this has not translated into significant production increases for Idaho or Montana as a result (Smith, 1999).

Table 16. Selected events affecting regional lead production

\begin{tabular}{|c|c|c|}
\hline Year & Event & Reference \\
\hline 1917 & Bunker Hill Smelter begins operation & Chapman, 1994 \\
\hline 1925 & Most mills now use flotation & $\begin{array}{l}\text { Bookstrom, } 2004 \text { written } \\
\text { commun. }\end{array}$ \\
\hline 1930 & $\begin{array}{l}\text { Severe curtailment of mining operations due to collapse of metals } \\
\text { prices }\end{array}$ & this study \\
\hline 1937 & Bunker Hill last mine to convert to all-through flotation & $\begin{array}{l}\text { Bookstrom, } 2004 \text { written } \\
\text { commun. }\end{array}$ \\
\hline 1942 & Expansion of mining operations for war effort & this study \\
\hline 1945 & Production drops off due to wartime labor shortage & this study \\
\hline 1949 & $\begin{array}{l}\text { Strike at Bunker Hill caused smelter shutdown for } 15 \text { days, after } \\
50 \text { years continuous production }\end{array}$ & Chapman, 1994 \\
\hline 1955 & Lucky Friday mine opened & Box, 2004 written commun. \\
\hline 1960 & 200-day long strike at Bunker Hill; other mines closed by strikes & Chapman, 1994 \\
\hline 1981 & Bunker Hill mine, mill, and smelter closed & Chapman, 1994 \\
\hline 1982 & Star-Morning mine closed & Box, 2004 written commun. \\
\hline 1983 & Bunker Hill smelter listed on EPA's NPL & $\begin{array}{l}\text { Bookstrom, } 2004 \text { written } \\
\text { commun. }\end{array}$ \\
\hline 1997 & Hecla begins production from Gold Hunter via Lucky Friday mine & this study \\
\hline
\end{tabular}

Table 17. Selected events affecting U.S. lead production

\begin{tabular}{lll}
\hline Year & Event & Reference \\
\hline 1906 & Froth flotation comes into widespread use & Kramer, undated \\
1917 & Lead used in lead-acid batteries & Kramer, undated \\
1960 & Production begins at Viburnum Trend & Kramer, undated \\
$1961-69$ & Lead and Zinc Mining Stabilization Program in effect & Smith, 1999 \\
1962 & Prolonged labor strike; production curtailed & Smith, 1999 \\
1973 & Lead in paint banned; phase-out of leaded gasoline & Kramer, undated \\
$1976-79$ & Post-Vietnam War boom & Smith, 1999 \\
$1992-96$ & Increasing demand for lead-acid batteries & Smith, 1999 \\
\hline
\end{tabular}



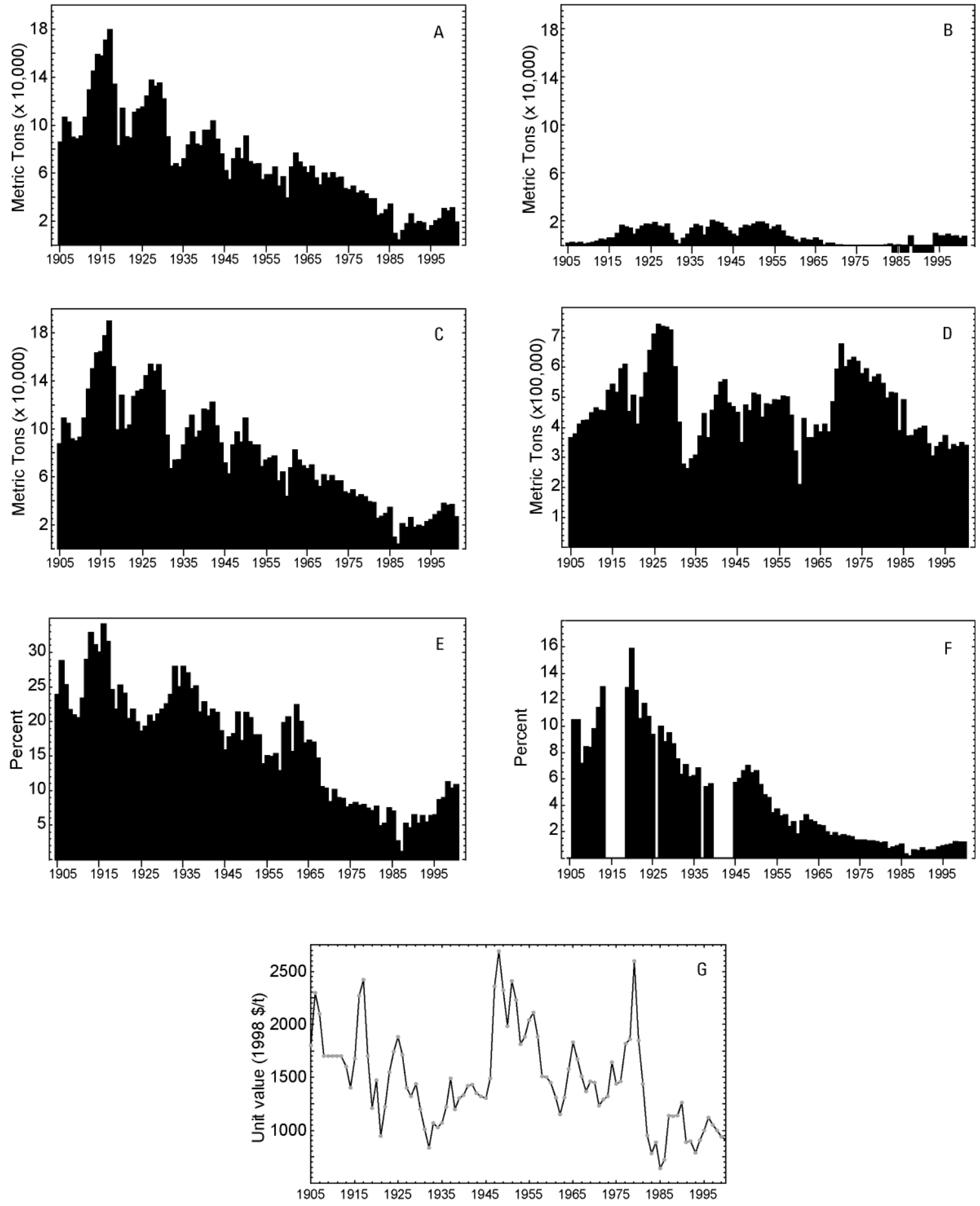

Figure 8. Lead production information from 1905-2001. A, annual production from Idaho. B, annual production from Montana. C, Idaho and Montana combined production. D, annual U.S. production. E, the proportion of regional lead production to U.S. production. $F$, the proportion of regional lead production to world production. $G$, the historical value of lead in constant 1998 dollars. Bars extending below the $\mathrm{x}$-axis represent data withheld from publication. 
Table 18. Lead production from Idaho and Montana, the U.S., and the world, 1905-2001

[The table also summarizes the proportions of Idaho and Montana production relative to the US and the world as well as the price for lead in constant 1998 dollars. Primary US production data represent the amount of refined lead. World production data for the years 1905-54 represent smelter production, while data for 1955-2000 represent mine production. Data for state, US, and world production are given in metric tons; proportions of US and world production by Idaho and Montana are given in percent; lead value is in US dollars. W, withheld; n.d., no data; leaders ( $\_$- ), no production]

Production

\begin{tabular}{|c|c|c|c|c|c|c|c|c|}
\hline \multirow[b]{2}{*}{ Year } & \multicolumn{5}{|l|}{ Production } & \multicolumn{2}{|c|}{ roduction to: } & \multirow[b]{2}{*}{$\begin{array}{r}\text { Lead } \\
\text { value } \\
(1998 \$ / t) \\
\end{array}$} \\
\hline & Idaho & Montana & $\begin{array}{l}\text { Idaho and } \\
\text { Montana } \\
\text { combined }\end{array}$ & US & World & US & World & \\
\hline 1905 & 85,346 & 1,902 & 87,248 & 366,000 & n.d. & 23.84 & n.d. & 1,800 \\
\hline 1906 & 106,249 & 2,254 & 108,503 & 378,000 & $1,040,000$ & 28.70 & 10.43 & 2,300 \\
\hline 1907 & 102,123 & 1,846 & 103,969 & 410,000 & 993,000 & 25.36 & 10.47 & 2,100 \\
\hline 1908 & 89,327 & 2,105 & 91,431 & 422,000 & $1,280,000$ & 21.67 & 7.14 & 1,700 \\
\hline 1909 & 88,164 & 1,248 & 89,413 & 426,000 & $1,060,000$ & 20.99 & 8.44 & 1,700 \\
\hline 1910 & 90,651 & 1,624 & 92,275 & 449,000 & $1,100,000$ & 20.55 & 8.39 & 1,700 \\
\hline 1911 & 106,287 & 2,267 & 108,554 & 465,000 & $1,110,000$ & 23.34 & 9.78 & 1,700 \\
\hline 1912 & 128,907 & 3,378 & 132,284 & 457,000 & $1,160,000$ & 28.95 & 11.40 & 1,700 \\
\hline 1913 & 144,187 & 4,961 & 149,147 & 455,000 & $1,150,000$ & 32.78 & 12.97 & 1,600 \\
\hline 1914 & 158,091 & 4,380 & 162,471 & 524,000 & n.d. & 31.01 & n.d. & 1,400 \\
\hline 1915 & 156,946 & 6,240 & 163,185 & 544,000 & n.d. & 30.00 & n.d. & 1,680 \\
\hline 1916 & 170,137 & 6,167 & 176,304 & 517,000 & n.d. & 34.10 & n.d. & 2,270 \\
\hline 1917 & 178,519 & 9,957 & 188,476 & 597,000 & n.d. & 31.57 & n.d. & 2,420 \\
\hline 1918 & 133,674 & 16,845 & 150,519 & 611,000 & n.d. & 24.63 & n.d. & 1,700 \\
\hline 1919 & 82,710 & 15,621 & 98,331 & 452,000 & 764,000 & 21.75 & 12.87 & 1,210 \\
\hline 1920 & 113,223 & 14,177 & 127,400 & 506,000 & 804,000 & 25.18 & 15.85 & 1,470 \\
\hline 1921 & 90,026 & 9,238 & 99,264 & 412,000 & 783,000 & 24.09 & 12.68 & 945 \\
\hline 1922 & 88,830 & 13,503 & 102,333 & 501,000 & 972,000 & 20.43 & 10.53 & 1,220 \\
\hline 1923 & 109,933 & 16,305 & 126,238 & 581,000 & $1,080,000$ & 21.73 & 11.69 & 1,550 \\
\hline 1924 & 112,924 & 17,906 & 130,830 & 657,000 & $1,220,000$ & 19.91 & 10.72 & 1,740 \\
\hline 1925 & 114,780 & 17,024 & 131,803 & 711,000 & $1,410,000$ & 18.54 & 9.35 & 1,880 \\
\hline 1926 & 123,824 & 19,190 & 143,014 & 744,000 & n.d. & 19.22 & n.d. & 1,710 \\
\hline 1927 & 137,004 & 16,283 & 153,288 & 736,000 & $1,540,000$ & 20.83 & 9.95 & 1,400 \\
\hline 1928 & 131,837 & 15,314 & 147,151 & 735,000 & $1,680,000$ & 20.02 & 8.76 & 1,320 \\
\hline 1929 & 134,896 & 17,787 & 152,684 & 724,000 & $1,610,000$ & 21.09 & 9.48 & 1,440 \\
\hline 1930 & 121,617 & 9,664 & 131,282 & 603,000 & $1,520,000$ & 21.77 & 8.64 & 1,200 \\
\hline 1931 & 90,144 & 4,019 & 94,163 & 419,000 & $1,260,000$ & 22.47 & 7.47 & 1,010 \\
\hline 1932 & 65,425 & 979 & 66,404 & 278,000 & $1,050,000$ & 23.89 & 6.32 & 833 \\
\hline 1933 & 67,462 & 5,971 & 73,433 & 263,000 & $1,040,000$ & 27.92 & 7.06 & 1,070 \\
\hline 1934 & 64,705 & 9,077 & 73,782 & 295,000 & $1,200,000$ & 25.01 & 6.15 & 1,030 \\
\hline 1935 & 71,687 & 14,142 & 85,829 & 308,000 & $1,380,000$ & 27.87 & 6.22 & 1,070 \\
\hline 1936 & 82,863 & 17,290 & 100,153 & 371,000 & $1,470,000$ & 27.00 & 6.81 & 1,220 \\
\hline 1937 & 94,087 & 16,291 & 110,377 & 447,000 & n.d. & 24.69 & n.d. & 1,490 \\
\hline 1938 & 83,623 & 8,461 & 92,084 & 367,000 & $1,700,000$ & 25.09 & 5.42 & 1,200 \\
\hline 1939 & 82,538 & 15,019 & 97,557 & 457,000 & $1,740,000$ & 21.35 & 5.61 & 1,300 \\
\hline 1940 & 95,105 & 20,898 & 116,004 & 508,000 & n.d. & 22.84 & n.d. & 1,330 \\
\hline 1941 & 95,178 & 19,286 & 114,464 & 551,000 & n.d. & 20.77 & n.d. & 1,420 \\
\hline 1942 & 103,338 & 18,189 & 121,528 & 558,000 & n.d. & 21.78 & n.d. & 1,430 \\
\hline 1943 & 87,506 & 14,809 & 102,315 & 481,000 & n.d. & 21.27 & n.d. & 1,350 \\
\hline
\end{tabular}




\begin{tabular}{|c|c|c|c|c|c|c|c|c|}
\hline 1944 & 75,778 & 11,889 & 87,667 & 470,000 & n.d. & 18.65 & n.d. & 1,320 \\
\hline 1945 & 62,095 & 9,071 & 71,166 & 450,000 & $1,250,000$ & 15.81 & 5.69 & 1,300 \\
\hline 1946 & 54,420 & 7,512 & 61,932 & 350,000 & $1,030,000$ & 17.69 & 6.01 & 1,490 \\
\hline 1947 & 71,618 & 14,613 & 86,231 & 474,000 & $1,310,000$ & 18.19 & 6.58 & 2,360 \\
\hline 1948 & 80,327 & 16,702 & 97,030 & 455,000 & $1,380,000$ & 21.33 & 7.03 & 2,690 \\
\hline 1949 & 71,940 & 16,326 & 88,266 & 515,000 & $1,370,000$ & 17.14 & 6.44 & 2,320 \\
\hline 1950 & 90,743 & 17,797 & 108,539 & 510,000 & $1,640,000$ & 21.28 & 6.62 & 1,980 \\
\hline 1951 & 69,594 & 19,325 & 88,919 & 434,000 & $1,600,000$ & 20.49 & 5.56 & 2,410 \\
\hline 1952 & 66,878 & 19,304 & 86,182 & 478,000 & $1,810,000$ & 18.03 & 4.76 & 2,230 \\
\hline 1953 & 67,686 & 18,098 & 85,784 & 477,000 & $1,870,000$ & 17.98 & 4.59 & 1,810 \\
\hline 1954 & 54,706 & 13,445 & 68,151 & 493,000 & $2,000,000$ & 13.82 & 3.41 & 1,880 \\
\hline 1955 & 58,209 & 15,448 & 73,656 & 490,000 & $2,010,000$ & 15.03 & 3.66 & 2,040 \\
\hline 1956 & 58,352 & 16,912 & 75,264 & 504,000 & $2,400,000$ & 14.93 & 3.14 & 2,110 \\
\hline 1957 & 64,989 & 12,066 & 77,055 & 502,000 & $2,380,000$ & 15.35 & 3.24 & 1,880 \\
\hline 1958 & 48,629 & 7,651 & 56,280 & 441,000 & $2,350,000$ & 12.76 & 2.39 & 1,510 \\
\hline 1959 & 56,605 & 6,960 & 63,565 & 321,000 & $2,320,000$ & 19.80 & 2.74 & 1,500 \\
\hline 1960 & 38,925 & 4,426 & 43,351 & 210,000 & $2,390,000$ & 20.64 & 1.81 & 1,450 \\
\hline 1961 & 64,843 & 2,398 & 67,241 & 430,000 & $2,390,000$ & 15.64 & 2.81 & 1,310 \\
\hline 1962 & 76,257 & 5,553 & 81,810 & 366,000 & $2,510,000$ & 22.35 & 3.26 & 1,150 \\
\hline 1963 & 68,729 & 4,536 & 73,265 & 366,000 & $2,560,000$ & 20.02 & 2.86 & 1,310 \\
\hline 1964 & 64,694 & 4,117 & 68,811 & 408,000 & $2,530,000$ & 16.87 & 2.72 & 1,580 \\
\hline 1965 & 60,425 & 6,333 & 66,758 & 385,000 & $2,700,000$ & 17.34 & 2.47 & 1,830 \\
\hline 1966 & 65,621 & 4,000 & 69,621 & 410,000 & $2,850,000$ & 16.98 & 2.44 & 1,670 \\
\hline 1967 & 55,690 & 815 & 56,505 & 386,000 & $2,870,000$ & 14.64 & 1.97 & 1,510 \\
\hline 1968 & 49,705 & 1,696 & 51,402 & 487,000 & $3,010,000$ & 10.55 & 1.71 & 1,370 \\
\hline 1969 & 59,510 & 1,590 & 61,100 & 594,000 & $3,240,000$ & 10.29 & 1.89 & 1,460 \\
\hline 1970 & 55,531 & 904 & 56,434 & 677,000 & $3,390,000$ & 8.34 & 1.66 & 1,450 \\
\hline 1971 & 60,429 & 558 & 60,987 & 604,000 & $3,490,000$ & 10.10 & 1.75 & 1,230 \\
\hline 1972 & 55,708 & 260 & 55,969 & 625,000 & $3,450,000$ & 8.96 & 1.62 & 1,290 \\
\hline 1973 & 56,014 & 160 & 56,174 & 633,000 & $3,490,000$ & 8.87 & 1.61 & 1,320 \\
\hline 1974 & 46,918 & 140 & 47,057 & 620,000 & $3,490,000$ & 7.59 & 1.35 & 1,640 \\
\hline 1975 & 45,718 & 186 & 45,904 & 579,000 & $3,440,000$ & 7.93 & 1.33 & 1,440 \\
\hline 1976 & 48,659 & 83 & 48,742 & 596,000 & $3,690,000$ & 8.18 & 1.32 & 1,460 \\
\hline 1977 & 42,872 & 96 & 42,968 & 552,000 & $3,410,000$ & 7.78 & 1.26 & 1,820 \\
\hline 1978 & 44,761 & 132 & 44,893 & 568,000 & $3,460,000$ & 7.90 & 1.30 & 1,860 \\
\hline 1979 & 42,636 & 258 & 42,894 & 578,000 & $3,510,000$ & 7.42 & 1.22 & 2,600 \\
\hline 1980 & 38,607 & 295 & 38,902 & 548,000 & $3,520,000$ & 7.10 & 1.11 & 1,850 \\
\hline 1981 & 38,397 & 194 & 38,591 & 498,000 & $3,350,000$ & 7.75 & 1.15 & 1,440 \\
\hline 1982 & 24,617 & 661 & 25,278 & 517,000 & $3,450,000$ & 4.89 & 0.73 & 949 \\
\hline 1983 & 25,893 & 1,163 & 27,056 & 515,000 & $3,350,000$ & 5.25 & 0.81 & 782 \\
\hline 1984 & 29,182 & W & 29,182 & 389,000 & $3,200,000$ & 7.50 & 0.91 & 885 \\
\hline 1985 & 33,707 & 846 & 34,553 & 494,000 & $3,390,000$ & 6.99 & 1.02 & 638 \\
\hline 1986 & 9,951 & W & 9,951 & 370,000 & $3,240,000$ & 2.69 & 0.31 & 721 \\
\hline 1987 & 4,126 & W & 4,126 & 373,000 & $3,430,000$ & 1.11 & 0.12 & 1,140 \\
\hline 1988 & 12,287 & 8,266 & 20,553 & 392,000 & $3,420,000$ & 5.24 & 0.60 & 1,130 \\
\hline 1989 & 17,957 & $\mathrm{~W}$ & 17,957 & 396,000 & $3,400,000$ & 4.53 & 0.53 & 1,140 \\
\hline 1990 & 25,895 & W & 25,895 & 404,000 & $3,370,000$ & 6.41 & 0.77 & 1,260 \\
\hline 1991 & 17,967 & W & 17,967 & 346,000 & $3,260,000$ & 5.19 & 0.55 & 884 \\
\hline 1992 & 19,356 & W & 19,356 & 305,000 & $3,200,000$ & 6.35 & 0.60 & 899 \\
\hline 1993 & 18,121 & W & 18,121 & 335,000 & $2,900,000$ & 5.41 & 0.62 & 788 \\
\hline
\end{tabular}




\begin{tabular}{rrrrrrrrr}
1994 & 12,269 & 9,940 & 22,209 & 351,000 & $2,800,000$ & 6.33 & 0.79 & 902 \\
1995 & 15,839 & 8,350 & 24,189 & 374,000 & $2,710,000$ & 6.47 & 0.89 & 998 \\
1996 & 20,179 & 7,970 & 28,149 & 326,000 & $2,920,000$ & 8.63 & 0.96 & 1,120 \\
1997 & 21,656 & 9,230 & 30,886 & 343,000 & $3,010,000$ & 9.00 & 1.03 & 1,050 \\
1998 & 30,580 & 7,310 & 37,890 & 337,000 & $3,100,000$ & 11.24 & 1.22 & 999 \\
1999 & 28,210 & 7,950 & 36,160 & 350,000 & $3,020,000$ & 10.33 & 1.20 & 942 \\
2000 & 30,972 & 6,020 & 36,992 & 341,000 & $3,100,000$ & 10.85 & 1.19 & 910 \\
2001 & 19,036 & 7,290 & 26,326 & n.d & n.d & n.d & n.d & n.d \\
\hline
\end{tabular}




\section{Lime}

Lime production in Idaho is largely withheld, though the few years that the commodity was reported after 1961 show production between 50,000 and 100,000 metric tons, with a peak in 1991 of 136,000 metric tons (fig. 9A). Montana shows an overall greater production capability by year, though much of the data is also withheld from publication (fig. 9B). When taken together, the Idaho and Montana charts provide a greater range of reporting years; however, because there are so many years of withheld information, the combined production is not accurately portrayed (fig. 9C). A change in lime production reporting was implemented in 1953 to include all captive lime but was not fully accomplished until 1961 (Kelly and others, 2001). As a result, lime production appears to double, even though no significant increases in production occurred. Figure 9D illustrates the increasing importance of lime based on rising production in the United States, especially in recent years. However, these trends are not observed in Idaho and Montana recorded production; the two states rarely exceed 1.2 percent of U.S. lime production (fig. 9E) and .25 percent of world production (fig. 9F). Furthermore, the value of lime has declined rapidly since 1979 and is currently at its lowest point of the last hundred years (fig. 9G).

Table 15. Selected events affecting U.S. lime production

\begin{tabular}{lll}
\hline Year & Event & Reference \\
\hline 1955 & First steel production by basic-oxygen furnace & Kramer, undated \\
1970 & Clean Air Act introduced. Control of $\mathrm{SO}_{2}$ leads to large market for lime & Kramer, undated \\
\hline
\end{tabular}



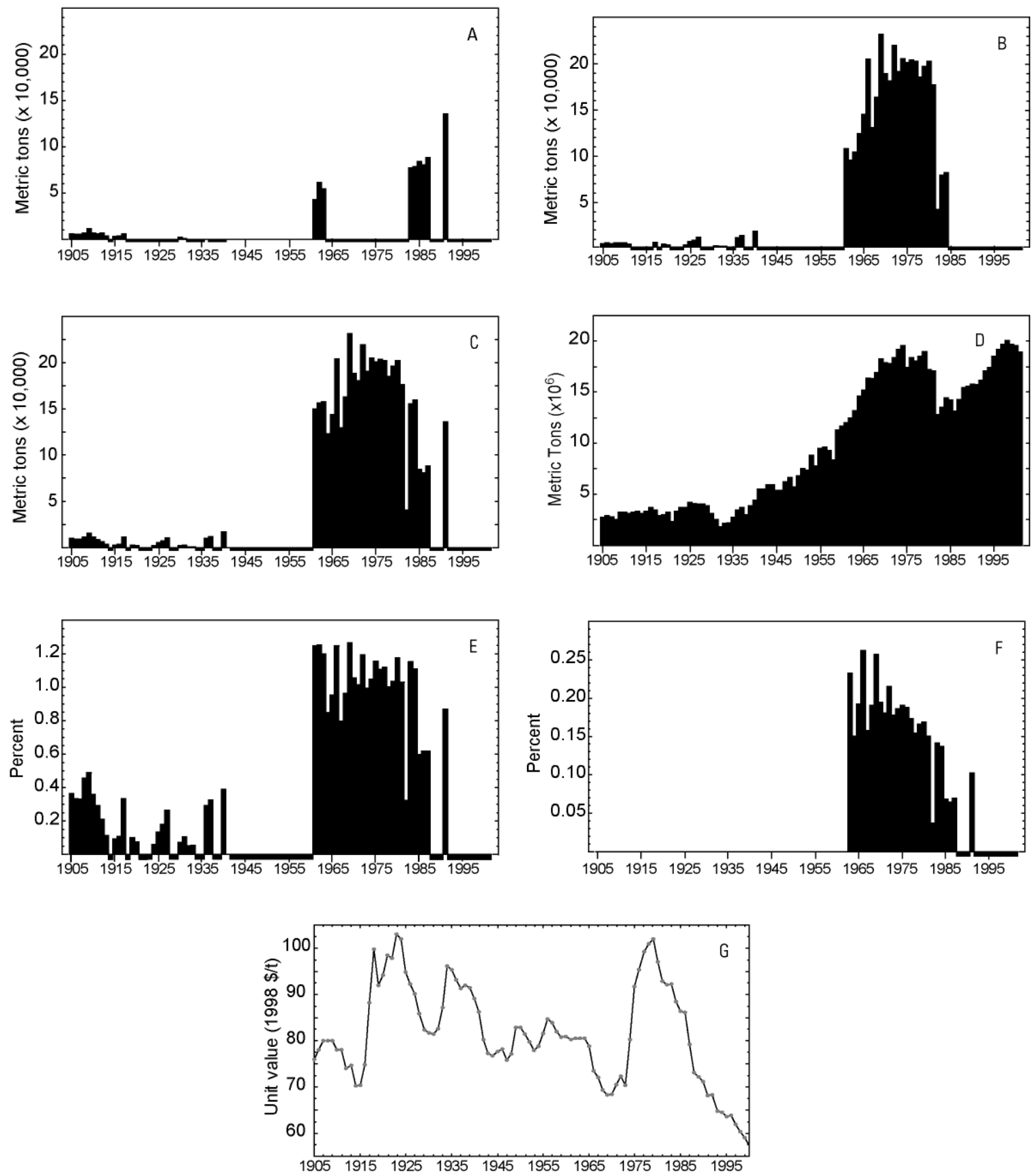

Figure 9. Lime production information from 1905-2001. A, annual production from Idaho. B, annual production from Montana. C, Idaho and Montana combined production. D, annual U.S. production in millions of metric tons. $E$, the proportion of regional lime production to U.S. production. F, the proportion of regional lime production to world production. $\mathrm{G}$, the historical value of lime in constant 1998 dollars. Bars extending below the $\mathrm{x}$-axis represent data withheld from publication. 
Table 20. Lime production from Idaho and Montana, the U.S., and the world, 1905-2001

[The table also summarizes the proportions of Idaho and Montana production relative to the US and the world as well as the price for lime in constant 1998 dollars. Data for state, US, and world production are given in metric tons; proportions of US and world production by Idaho and Montana are given in percent; lime value is in US dollars. W, withheld; n.d., no data; leaders ( _ - ), no production]

\begin{tabular}{|c|c|c|c|c|c|c|c|c|}
\hline \multirow[b]{2}{*}{ Year } & \multicolumn{4}{|c|}{ Production } & \multicolumn{3}{|c|}{$\begin{array}{l}\text { Proportion of Idaho } \\
\text { and Montana } \\
\text { production to: }\end{array}$} & \multirow[b]{2}{*}{$\begin{array}{r}\text { Lime value } \\
(1998 \text { \$/t) }\end{array}$} \\
\hline & Idaho & Montana & $\begin{array}{l}\text { Idaho and } \\
\text { Montana } \\
\text { combined } \\
\end{array}$ & US & World & US & World & \\
\hline 1905 & 6,073 & 3,695 & 9,768 & $2,700,000$ & n.d. & 0.36 & n.d. & 76.00 \\
\hline 1906 & 5,382 & 4,305 & 9,686 & $2,900,000$ & n.d. & 0.33 & n.d. & 78.00 \\
\hline 1907 & 5,411 & 3,826 & 9,236 & $2,800,000$ & n.d. & 0.33 & n.d. & 80.00 \\
\hline 1908 & 6,689 & 4,646 & 11,335 & $2,500,000$ & n.d. & 0.45 & n.d. & 80.00 \\
\hline 1909 & 11,459 & 4,119 & 15,578 & $3,200,000$ & n.d. & 0.49 & n.d. & 80.00 \\
\hline 1910 & 7,343 & 4,064 & 11,407 & $3,200,000$ & n.d. & 0.36 & n.d. & 78.00 \\
\hline 1911 & 6,177 & 2,812 & 8,989 & $3,100,000$ & n.d. & 0.29 & n.d. & 78.00 \\
\hline 1912 & 6,715 & W & 6,715 & $3,200,000$ & n.d. & 0.21 & n.d. & 74.00 \\
\hline 1913 & 3,749 & W & 3,749 & $3,300,000$ & n.d. & 0.11 & n.d. & 74.60 \\
\hline 1914 & W & W & W & $3,100,000$ & n.d. & W & n.d. & 70.20 \\
\hline 1915 & 3,045 & $\mathrm{~W}$ & 3,045 & $3,300,000$ & n.d. & 0.09 & n.d. & 70.40 \\
\hline 1916 & 3,982 & W & 3,982 & $3,700,000$ & n.d. & 0.11 & n.d. & 74.80 \\
\hline 1917 & 6,557 & 4,791 & 11,348 & $3,400,000$ & n.d. & 0.33 & n.d. & 88.20 \\
\hline 1918 & W & W & W & $2,900,000$ & n.d. & W & n.d. & 99.80 \\
\hline 1919 & W & 3,030 & 3,030 & $3,000,000$ & n.d. & 0.10 & n.d. & 92.00 \\
\hline 1920 & W & 2,393 & 2,393 & $3,200,000$ & n.d. & 0.07 & n.d. & 94.20 \\
\hline 1921 & W & W & W & $2,300,000$ & n.d. & $\mathrm{W}$ & n.d. & 98.50 \\
\hline 1922 & W & W & W & $3,300,000$ & n.d. & W & n.d. & 97.80 \\
\hline 1923 & W & W & W & $3,700,000$ & n.d. & W & n.d. & 103.00 \\
\hline 1924 & W & 2,154 & 2,154 & $3,700,000$ & n.d. & 0.06 & n.d. & 102.00 \\
\hline 1925 & W & 5,687 & 5,687 & $4,200,000$ & n.d. & 0.14 & n.d. & 94.90 \\
\hline 1926 & W & 7,347 & 7,347 & $4,100,000$ & n.d. & 0.18 & n.d. & 92.20 \\
\hline 1927 & W & 10,530 & 10,530 & $4,000,000$ & n.d. & 0.26 & n.d. & 90.20 \\
\hline 1928 & W & $\mathrm{W}$ & $\mathrm{W}$ & $4,000,000$ & n.d. & W & n.d. & 85.80 \\
\hline 1929 & W & W & W & $3,900,000$ & n.d. & $\mathrm{W}$ & n.d. & 82.30 \\
\hline 1930 & 2,234 & W & 2,234 & $3,100,000$ & n.d. & 0.07 & n.d. & 81.70 \\
\hline 1931 & 798 & 1,840 & 2,638 & $2,500,000$ & n.d. & 0.11 & n.d. & 81.40 \\
\hline 1932 & W & 922 & 922 & $1,800,000$ & n.d. & 0.05 & n.d. & 82.60 \\
\hline 1933 & W & 1,135 & 1,135 & $2,100,000$ & n.d. & 0.05 & n.d. & 87.10 \\
\hline 1934 & W & W & W & $2,200,000$ & n.d. & W & n.d. & 96.10 \\
\hline 1935 & W & W & W & $2,700,000$ & n.d. & W & n.d. & 95.30 \\
\hline 1936 & -- & 9,945 & 9,945 & $3,400,000$ & n.d. & 0.29 & n.d. & 93.20 \\
\hline 1937 & $\mathrm{~W}$ & 12,061 & 12,061 & $3,700,000$ & n.d. & 0.33 & n.d. & 91.30 \\
\hline 1938 & W & $\mathrm{W}$ & $\mathrm{W}$ & $3,000,000$ & n.d. & W & n.d. & 92.00 \\
\hline 1939 & W & $\mathrm{W}$ & W & $3,900,000$ & n.d. & $\mathrm{W}$ & n.d. & 91.40 \\
\hline
\end{tabular}




\begin{tabular}{|c|c|c|c|c|c|c|c|c|}
\hline 1940 & W & 17,053 & 17,053 & $4,400,000$ & n.d. & 0.39 & n.d. & 89.10 \\
\hline 1941 & 109 & W & 109 & $5,500,000$ & n.d. & 0.00 & n.d. & 86.20 \\
\hline 1942 & - - & W & W & $5,500,000$ & n.d. & W & n.d. & 80.20 \\
\hline 1943 & -- & W & $\mathrm{W}$ & $5,900,000$ & n.d. & W & n.d. & 77.30 \\
\hline 1944 & -- & W & W & $5,900,000$ & n.d. & W & n.d. & 76.80 \\
\hline 1945 & -- & W & W & $5,400,000$ & n.d. & W & n.d. & 77.70 \\
\hline 1946 & -- & W & W & $5,400,000$ & n.d. & W & n.d. & 78.20 \\
\hline 1947 & -- & W & W & $6,200,000$ & n.d. & W & n.d. & 75.80 \\
\hline 1948 & -- & W & W & $6,600,000$ & n.d. & W & n.d. & 77.10 \\
\hline 1949 & -- & W & W & $5,700,000$ & n.d. & W & n.d. & 82.80 \\
\hline 1950 & -- & $\mathrm{W}$ & W & $6,800,000$ & n.d. & $\mathrm{W}$ & n.d. & 82.90 \\
\hline 1951 & _- & W & W & $7,500,000$ & n.d. & W & n.d. & 81.40 \\
\hline 1952 & -- & W & W & $7,300,000$ & n.d. & W & n.d. & 79.80 \\
\hline 1953 & -- & W & $\mathrm{W}$ & $8,800,000$ & n.d. & W & n.d. & 77.90 \\
\hline 1954 & -- & W & $\mathrm{W}$ & $7,800,000$ & n.d. & W & n.d. & 78.80 \\
\hline 1955 & -- & W & W & $9,500,000$ & n.d. & W & n.d. & 81.50 \\
\hline 1956 & -- & W & W & $9,600,000$ & n.d. & W & n.d. & 84.70 \\
\hline 1957 & -- & W & W & $9,300,000$ & n.d. & W & n.d. & 83.90 \\
\hline 1958 & -- & W & $\mathrm{W}$ & $8,400,000$ & n.d. & W & n.d. & 81.90 \\
\hline 1959 & _- & W & W & $11,300,000$ & n.d. & W & n.d. & 80.80 \\
\hline 1960 & -- & W & W & $11,700,000$ & n.d. & W & n.d. & 80.90 \\
\hline 1961 & 42,638 & 107,050 & 149,688 & $12,000,000$ & n.d. & 1.25 & n.d. & 80.20 \\
\hline 1962 & 61,690 & 94,349 & 156,038 & $12,500,000$ & n.d. & 1.25 & n.d. & 80.50 \\
\hline 1963 & 54,432 & 103,421 & 157,853 & $13,200,000$ & $68,000,000$ & 1.20 & 0.23 & 80.50 \\
\hline 1964 & $\mathrm{~W}$ & 123,379 & 123,379 & $14,600,000$ & $82,000,000$ & 0.85 & 0.15 & 80.50 \\
\hline 1965 & $\mathrm{~W}$ & 144,245 & 144,245 & $15,200,000$ & $75,000,000$ & 0.95 & 0.19 & 78.80 \\
\hline 1966 & W & 204,120 & 204,120 & $16,400,000$ & $78,000,000$ & 1.24 & 0.26 & 73.50 \\
\hline 1967 & W & 129,730 & 129,730 & $16,300,000$ & $82,000,000$ & 0.80 & 0.16 & 72.10 \\
\hline 1968 & W & 162,389 & 162,389 & $16,900,000$ & $85,000,000$ & 0.96 & 0.19 & 69.30 \\
\hline 1969 & W & 231,336 & 231,336 & $18,300,000$ & $90,000,000$ & 1.26 & 0.26 & 68.30 \\
\hline 1970 & W & 188,698 & 188,698 & $17,900,000$ & $97,000,000$ & 1.05 & 0.19 & 68.40 \\
\hline 1971 & W & 180,533 & 180,533 & $17,800,000$ & $100,000,000$ & 1.01 & 0.18 & 70.50 \\
\hline 1972 & W & 219,542 & 219,542 & $18,400,000$ & $102,000,000$ & 1.19 & 0.22 & 72.30 \\
\hline 1973 & W & 190,512 & 190,512 & $19,200,000$ & $107,000,000$ & 0.99 & 0.18 & 70.40 \\
\hline 1974 & W & 205,027 & 205,027 & $19,600,000$ & $110,000,000$ & 1.05 & 0.19 & 80.20 \\
\hline 1975 & W & 200,491 & 200,491 & $17,400,000$ & $105,000,000$ & 1.15 & 0.19 & 91.70 \\
\hline 1976 & W & 203,213 & 203,213 & $18,400,000$ & $108,000,000$ & 1.10 & 0.19 & 95.30 \\
\hline 1977 & W & 202,306 & 202,306 & $18,100,000$ & $117,000,000$ & 1.12 & 0.17 & 99.30 \\
\hline 1978 & W & 185,069 & 185,069 & $18,500,000$ & $120,000,000$ & 1.00 & 0.15 & 101.00 \\
\hline 1979 & W & 195,955 & 195,955 & $19,000,000$ & $118,000,000$ & 1.03 & 0.17 & 102.00 \\
\hline 1980 & W & 202,306 & 202,306 & $17,200,000$ & $120,000,000$ & 1.18 & 0.17 & 97.00 \\
\hline 1981 & W & 175,997 & 175,997 & $17,100,000$ & $117,000,000$ & 1.03 & 0.15 & 92.90 \\
\hline 1982 & W & 40,824 & 40,824 & $12,800,000$ & $109,000,000$ & 0.32 & 0.04 & 92.10 \\
\hline 1983 & 77,112 & 78,019 & 155,131 & $13,500,000$ & $110,000,000$ & 1.15 & 0.14 & 92.20 \\
\hline 1984 & 78,926 & 80,741 & 159,667 & $14,400,000$ & $117,000,000$ & 1.11 & 0.14 & 88.50 \\
\hline 1985 & 84,370 & W & 84,370 & $14,200,000$ & $123,000,000$ & 0.59 & 0.07 & 86.40 \\
\hline 1986 & 80,741 & W & 80,741 & $13,100,000$ & $124,000,000$ & 0.62 & 0.07 & 86.10 \\
\hline 1987 & 87,998 & W & 87,998 & $14,300,000$ & $127,000,000$ & 0.62 & 0.07 & 79.20 \\
\hline
\end{tabular}




\begin{tabular}{lrrrrrrrr}
1988 & W & W & W & $15,500,000$ & $134,000,000$ & W & W & 73.10 \\
1989 & W & W & W & $15,600,000$ & $139,000,000$ & W & W & 72.20 \\
1990 & W & W & W & $15,800,000$ & $136,000,000$ & W & W & 71.20 \\
1991 & 136,080 & W & 136,080 & $15,700,000$ & $133,000,000$ & 0.87 & 0.10 & 68.20 \\
1992 & W & W & W & $16,200,000$ & $133,000,000$ & W & W & 68.30 \\
1993 & W & W & W & $17,100,000$ & $123,000,000$ & W & W & 64.75 \\
1994 & W & W & W & $17,400,000$ & $118,000,000$ & W & W & 64.50 \\
1995 & W & W & W & $18,500,000$ & $120,000,000$ & W & W & 63.60 \\
1996 & W & W & W & $19,200,000$ & $121,000,000$ & W & W & 63.89 \\
1997 & W & W & W & $19,700,000$ & $118,000,000$ & W & W & 61.95 \\
1998 & W & W & W & $20,100,000$ & $117,000,000$ & W & W & 60.30 \\
1999 & W & W & W & $19,700,000$ & $116,000,000$ & W & W & 59.09 \\
2000 & W & W & W & $19,600,000$ & $118,000,000$ & W & W & 57.36 \\
2001 & W & W & W & $18,900,000$ & $118,000,000$ & W & W & 56.42 \\
\hline
\end{tabular}




\section{Mercury}

Idaho has had a volatile record of mercury production, which began in 1917 and ended in 1972 (fig. 10A). Idaho's Hermes mine produced 148 metric tons of mercury in 1943 to meet the high demand during WWII. Abundant mining operations remained after the war; however, production levels diminished and mercury mining was suspended after the commodity was declared a hazardous substance by the U.S. Environmental Protection Agency in 1971 (Kramer, undated). Idaho's last operating mercury mine, the Idaho-Almaden mine, shut down early in 1972. There has been no recorded mercury production in Idaho since the Idaho-Almaden mine closure. The state of Montana has never been a significant producer of mercury, and no production was reported in the USBM Yearbooks. Furthermore, no charts were produced for the proportion of regional mercury mining production to U.S. or world mercury production, due to the very small numbers involved.

Table 21. Selected events affecting regional mercury production

\begin{tabular}{lll}
\hline Year & Event & Reference \\
\hline $1942-1968$ & Years of operation of Hermes mine & this study \\
$1955-1972$ & Years of operation of Idaho-Almaden mine & this study \\
\hline
\end{tabular}

Table 22. Selected events affecting U.S. mercury production

\begin{tabular}{lll}
\hline Year & Event & Reference \\
\hline WWI \& WWII & High demand leads to increased prices & Reese, 1999 \\
\hline 1971 & $\begin{array}{l}\text { Environmental Protection Agency declares Mercury a } \\
\text { hazardous substance }\end{array}$ & Kramer, undated \\
& Last US primary mercury mine closed & Kramer, undated \\
\hline
\end{tabular}
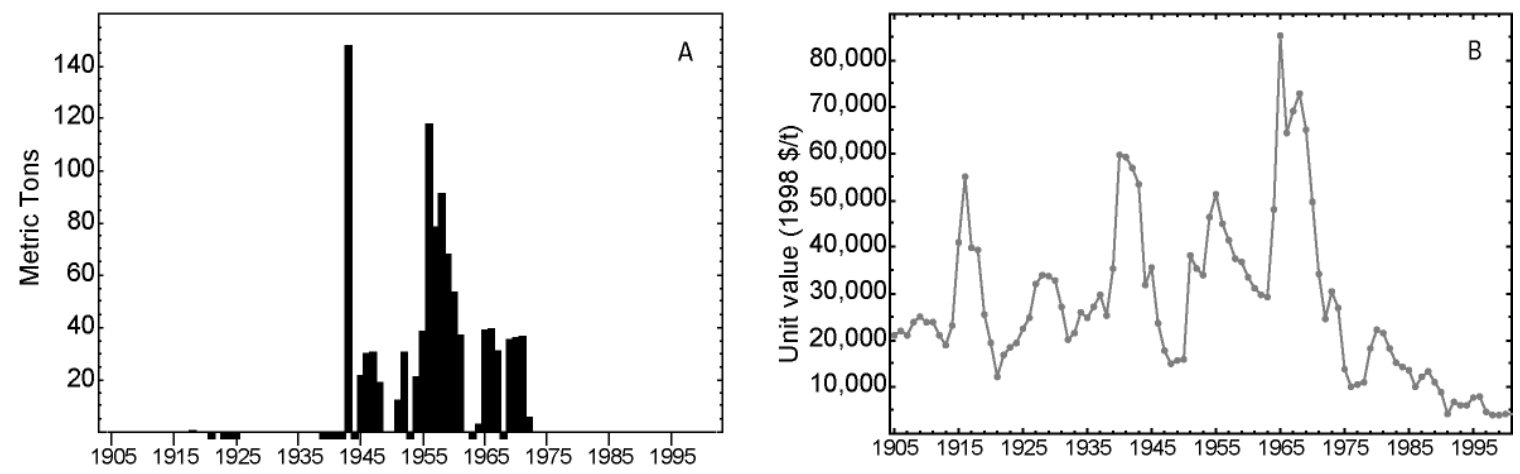

Figure 10. Mercury production information from 1905-2001. A, annual production from Idaho. $B$, the historical value of mercury in constant 1998 dollars. Bars extending below the x-axis represent data withheld from publication. 
Table 23. Mercury production from Idaho, the U.S., and the world, 1905-2001

[The table also summarizes the proportions of Idaho production relative to the US and the world as well as the price for mercury in constant 1998 dollars. Figures for national mercury production reflect domestic mine production and the mercury byproduct recovered from gold, copper, and zinc mining. Production data for the world represent mine production of mercury. Data for state, US, and world production are given in metric tons; proportions of US and world production by Idaho are given in percent; mercury value is in US dollars. W, withheld; n.d., no data; leaders $(\ldots$, ), no production]

\begin{tabular}{|c|c|c|c|c|c|c|}
\hline \multirow[b]{2}{*}{ Year } & \multicolumn{3}{|c|}{ Production } & \multicolumn{2}{|c|}{ Proportion of Idaho production to: } & \multirow{2}{*}{$\begin{array}{r}\text { Mercury value } \\
(1998 \$ / \mathrm{t})\end{array}$} \\
\hline & Idaho & US & World & US & World & \\
\hline 1905 & -- & 1,040 & 3,340 & -- & -- & 21,000 \\
\hline 1906 & -- & 887 & 3,860 & -- & -- & 22,000 \\
\hline 1907 & -- & 733 & 3,310 & -- & -- & 21,000 \\
\hline 1908 & -- & 672 & 3,300 & -- & -- & 24,000 \\
\hline 1909 & - - & 717 & 3,230 & - - & - - & 25,000 \\
\hline 1910 & - - & 701 & 3,690 & - - & - - & 24,000 \\
\hline 1911 & -- & 723 & 4,250 & -- & -- & 24,000 \\
\hline 1912 & -- & 853 & 4,160 & -- & -- & 21,000 \\
\hline 1913 & -- & 688 & 4,050 & -- & -- & 19,100 \\
\hline 1914 & -- & 563 & 3,740 & -- & -- & 23,100 \\
\hline 1915 & -- & 716 & 3,890 & -- & -- & 41,100 \\
\hline 1916 & -- & 1,020 & 3,500 & -- & -- & 55,100 \\
\hline 1917 & 0.17 & 1,230 & 3,970 & 0.00 & 0.00 & 39,800 \\
\hline 1918 & 0.76 & 1,120 & 3,420 & 0.00 & 0.00 & 39,300 \\
\hline 1919 & - - & 729 & 3,100 & - - & -- & 25,600 \\
\hline 1920 & -- & 456 & 2,910 & - - & -- & 19,400 \\
\hline 1921 & W & 216 & 2,130 & W & W & 12,100 \\
\hline 1922 & -- & 217 & 3,170 & -- & -- & 16,800 \\
\hline 1923 & W & 270 & 3,210 & W & W & 18,600 \\
\hline 1924 & W & 343 & 3,070 & W & W & 19,500 \\
\hline 1925 & W & 312 & 3,560 & W & W & 22,600 \\
\hline 1926 & 0.21 & 260 & 4,000 & 0.00 & 0.00 & 24,800 \\
\hline 1927 & - - & 384 & 5,170 & -- & - - & 32,000 \\
\hline 1928 & -- & 616 & 5,140 & -- & -- & 34,100 \\
\hline 1929 & - - & 816 & 5,610 & - - & -- & 33,700 \\
\hline 1930 & -- & 743 & 3,760 & - - & -- & 32,700 \\
\hline 1931 & -- & 860 & 3,420 & -- & -- & 27,100 \\
\hline 1932 & -- & 435 & 2,850 & -- & -- & 20,100 \\
\hline 1933 & -- & 333 & 2,060 & -- & -- & 21,600 \\
\hline 1934 & -- & 532 & 2,650 & -- & -- & 26,100 \\
\hline 1935 & -- & 604 & 3,460 & -- & -- & 24,800 \\
\hline 1936 & -- & 571 & 4,270 & -- & -- & 27,300 \\
\hline 1937 & -- & 569 & 4,590 & -- & -- & 29,700 \\
\hline 1938 & -- & 620 & 5,170 & -- & -- & 25,300 \\
\hline 1939 & W & 642 & 4,830 & W & W & 35,400 \\
\hline 1940 & W & 1,300 & 7,130 & W & W & 59,700 \\
\hline 1941 & W & 1,550 & 9,170 & W & W & 59,400 \\
\hline 1942 & W & 1,750 & 8,990 & W & W & 57,000 \\
\hline 1943 & 147.86 & 1,790 & 7,870 & 0.08 & 0.02 & 53,400 \\
\hline
\end{tabular}




\begin{tabular}{|c|c|c|c|c|c|c|}
\hline 1944 & W & 1,300 & 5,330 & W & W & 31,800 \\
\hline 1945 & 21.76 & 1,060 & 4,180 & 0.02 & 0.01 & 35,600 \\
\hline 1946 & 30.12 & 874 & 4,960 & 0.03 & 0.01 & 23,700 \\
\hline 1947 & 30.74 & 801 & 5,360 & 0.04 & 0.01 & 17,700 \\
\hline 1948 & 18.84 & 496 & 3,260 & 0.04 & 0.01 & 15,000 \\
\hline 1949 & -- & 342 & 4,160 & -- & -- & 15,800 \\
\hline 1950 & -- & 156 & 4,940 & -- & -- & 15,900 \\
\hline 1951 & 12.39 & 251 & 5,060 & 0.05 & 0.00 & 38,300 \\
\hline 1952 & 30.78 & 433 & 5,190 & 0.07 & 0.01 & 35,400 \\
\hline 1953 & W & 494 & 5,510 & W & W & 34,100 \\
\hline 1954 & 21.13 & 639 & 6,180 & 0.03 & 0.00 & 46,500 \\
\hline 1955 & 38.41 & 653 & 6,380 & 0.06 & 0.01 & 51,400 \\
\hline 1956 & 117.77 & 833 & 7,620 & 0.14 & 0.02 & 45,100 \\
\hline 1957 & 78.42 & 1,190 & 8,260 & 0.07 & 0.01 & 41,400 \\
\hline 1958 & 91.09 & 1,310 & 8,490 & 0.07 & 0.01 & 37,500 \\
\hline 1959 & 68.05 & 1,080 & 7,710 & 0.06 & 0.01 & 36,900 \\
\hline 1960 & 53.37 & 1,150 & 8,340 & 0.05 & 0.01 & 33,600 \\
\hline 1961 & 37.23 & 1,090 & 8,260 & 0.03 & 0.00 & 31,200 \\
\hline 1962 & -- & 906 & 8,430 & -- & -- & 29,800 \\
\hline 1963 & W & 659 & 8,260 & W & W & 29,200 \\
\hline 1964 & 2.88 & 488 & 8,800 & 0.01 & 0.00 & 48,100 \\
\hline 1965 & 38.83 & 675 & 9,230 & 0.06 & 0.00 & 85,300 \\
\hline 1966 & 39.35 & 759 & 9,140 & 0.05 & 0.00 & 64,400 \\
\hline 1967 & 31.16 & 820 & 8,000 & 0.04 & 0.00 & 69,200 \\
\hline 1968 & W & 995 & 8,950 & W & W & 72,900 \\
\hline 1969 & 35.12 & 1,020 & 9,970 & 0.03 & 0.00 & 65,140 \\
\hline 1970 & 36.02 & 941 & 9,790 & 0.04 & 0.00 & 49,660 \\
\hline 1971 & 36.68 & 616 & 10,400 & 0.06 & 0.00 & 34,150 \\
\hline 1972 & 5.59 & 253 & 9,620 & 0.02 & 0.00 & 24,680 \\
\hline 1973 & -- & 76.8 & 9,250 & -- & -- & 30,460 \\
\hline 1974 & -- & 75.5 & 8,880 & -- & -- & 27,010 \\
\hline 1975 & -- & 254 & 8,700 & -- & -- & 13,900 \\
\hline 1976 & -- & 797 & 8,090 & -- & -- & 10,080 \\
\hline 1977 & -- & 974 & 6,580 & -- & -- & 10,590 \\
\hline 1978 & -- & 833 & 6,250 & -- & -- & 11,110 \\
\hline 1979 & -- & 1,020 & 6,010 & -- & -- & 18,320 \\
\hline 1980 & -- & 1,060 & 6,810 & -- & -- & 22,350 \\
\hline 1981 & -- & 962 & 7,270 & -- & -- & 21,520 \\
\hline 1982 & -- & 888 & 6,820 & -- & -- & 18,170 \\
\hline 1983 & -- & 864 & 6,230 & -- & -- & 15,310 \\
\hline 1984 & -- & 657 & 6,740 & -- & -- & 14,310 \\
\hline 1985 & -- & 570 & 6,140 & -- & -- & 13,670 \\
\hline 1986 & -- & 483 & 7,780 & -- & -- & 10,040 \\
\hline 1987 & -- & 34 & 5,530 & -- & -- & 12,300 \\
\hline 1988 & -- & 379 & 6,840 & -- & -- & 13,410 \\
\hline 1989 & -- & 414 & 6,750 & -- & -- & 10,980 \\
\hline 1990 & -- & 562 & 4,100 & -- & -- & 9,019 \\
\hline 1991 & -- & 58 & 2,540 & -- & -- & 4,250 \\
\hline 1992 & -- & 64 & 1,960 & -- & -- & 6,787 \\
\hline 1993 & -- & n.d. & 1,730 & -- & -- & 6,106 \\
\hline
\end{tabular}




\begin{tabular}{lllllll}
1994 & -- & n.d. & 1,960 & -- & -- & 6,203 \\
1995 & -- & n.d. & 3,190 & -- & -- & 7,676 \\
1996 & -- & n.d. & 2,560 & -- & -- & 7,887 \\
1997 & -- & n.d. & 2,410 & -- & -- & 4,700 \\
1998 & -- & n.d. & 1,580 & -- & -- & 4,057 \\
1999 & -- & n.d. & 1,310 & -- & -- & 3,973 \\
2000 & -- & n.d. & 1,350 & -- & -- & 4,256 \\
2001 & -- & n.d. & 1,490 & -- & -- & 4,138 \\
\hline
\end{tabular}




\section{Platinum Group Elements (PGE)}

The Stillwater mine in Nye, Montana began operations in 1986 and became the nation's first major PGE mine (Kramer, undated). Early platinum production at Stillwater was 1.5 to 2 metric tons per year, but production increased to more than 3.5 metric tons by 2001 (fig. 11A). Palladium mining has had similar trends in production, though the more plentiful metal has been produced at a rate of about three times that of platinum (fig. 11B). Combined platinum and palladium production in Montana has greatly increased from 1997 to 2001, nearly doubling from earlier production rates (fig. 11C). Prior to the opening of the Stillwater mine in Montana, the U.S. produced between one-half and 2 metric tons of PGE minerals each year from placer platinum deposits in Alaska (fig. 11D). In 1950, national production reporting began to include the PGE minerals obtained as byproducts of gold and copper smelting (Kelley and others, 2001). Moreover, the large contribution of PGE commodities from Montana supplied between two and four percent of the world's PGE production every year since 1987 (fig. 11F). The Stillwater mine was the only PGE mine in the U.S. from 1987 to 2002 when the East Boulder mine began operations on the PGE deposit as well. Montana platinum and palladium production equals that reported for the U.S. after 1986; however, PGE production reported for Montana in USBM yearbooks and reported for the U.S. in Kelly and others (2001) do not agree for the years 1992 and 1993, where 100 percent of the nations PGE minerals should have originated in Montana.

Table 24. Selected events affecting regional PGE production

\begin{tabular}{lll}
\hline Year & Event & Reference \\
\hline 1986 & Stillwater mine in Mont. opens. 1987 first year of production & Kramer, undated \\
2002 & East Boulder mine opens & this study \\
\hline
\end{tabular}

Table 25. Selected events affecting U.S. PGE production

\begin{tabular}{lll}
\hline Year & Event & Reference \\
\hline 1923 & Platinum lodes discovered in Transvaal, Republic of South Africa & Page and others, 1973 \\
1924 & Bushveld Complex discovered & Page and others, 1973 \\
1924 & Platinum placers discovered in Alaska - becomes 5 $5^{\text {th }}$ largest producer & Page and others, 1973 \\
& in world by 1934. & \\
$1957-1958$ & Drop in demand by domestic petroleum refineries & Hilliard, 1999a \\
1975 & Catalytic converters required in autos & Kramer, undated \\
1988 & Ford Motor Company announces platinum-free catalytic converter & Hilliard, 1999a \\
\hline
\end{tabular}



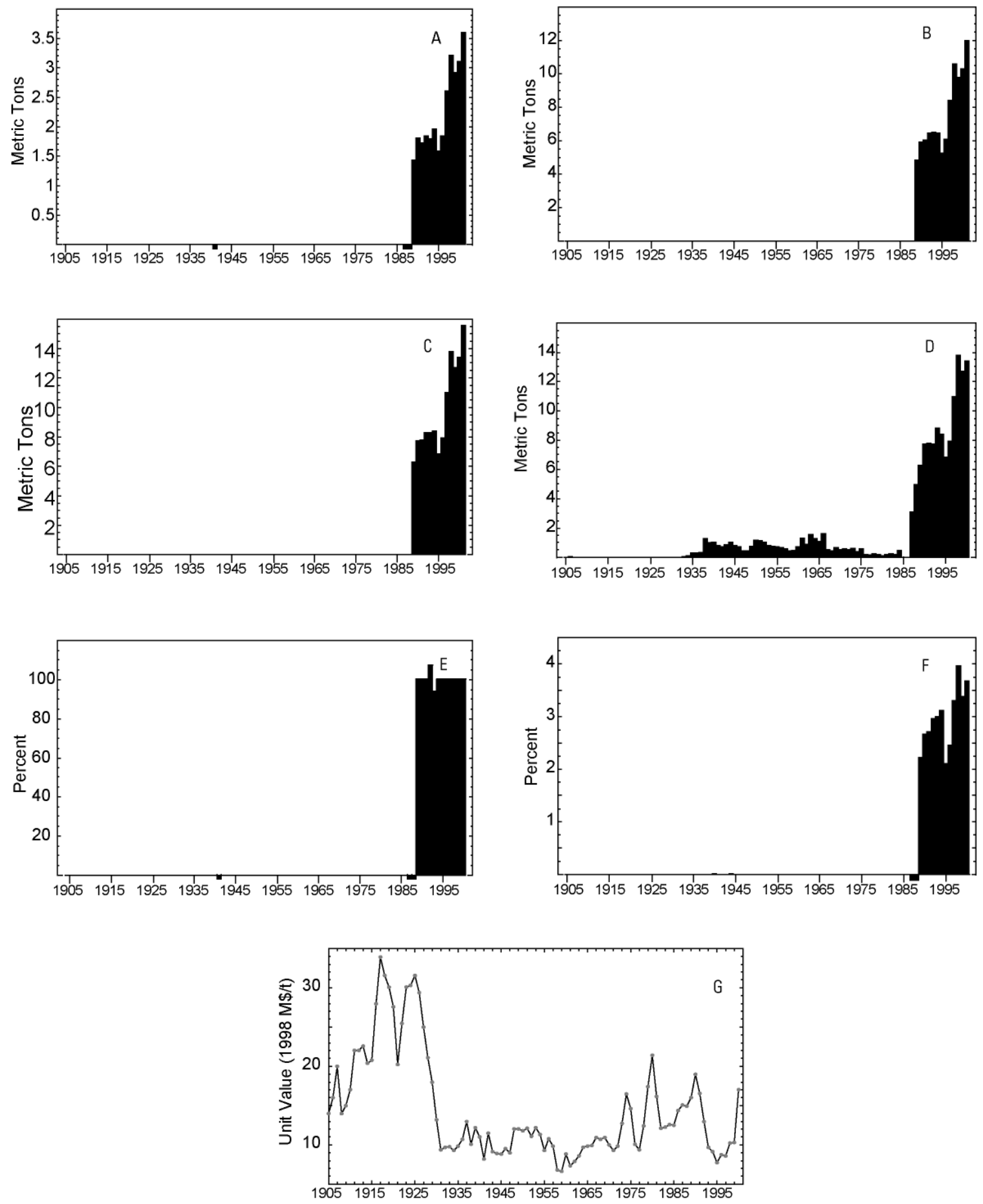

Figure 11. PGE production information from 1905-2001. A, annual platinum production from Montana. B, annual palladium production from Montana. C, combined platinum and palladium production. D, annual U.S. PGE production. $\mathrm{E}$, the proportion of regional PGE production to U.S. production. $F$, the proportion of regional PGE production to world production. $\mathrm{G}$, the historical value of platinum in constant 1998 dollars. Bars extending below the x-axis represent data withheld from publication. 
Table 26. PGE production from Montana, the U.S., and the world, 1905-2001

[The table also summarizes the proportions of Montana production relative to the US and the world as well as the price for PGE in constant 1998 dollars. Data for state, US, and world production are given in metric tons; proportions of US and world production by Idaho and Montana are given in percent; PGE value is in US dollars. W, withheld; n.d., no data; leaders ( _ _ ), no production]

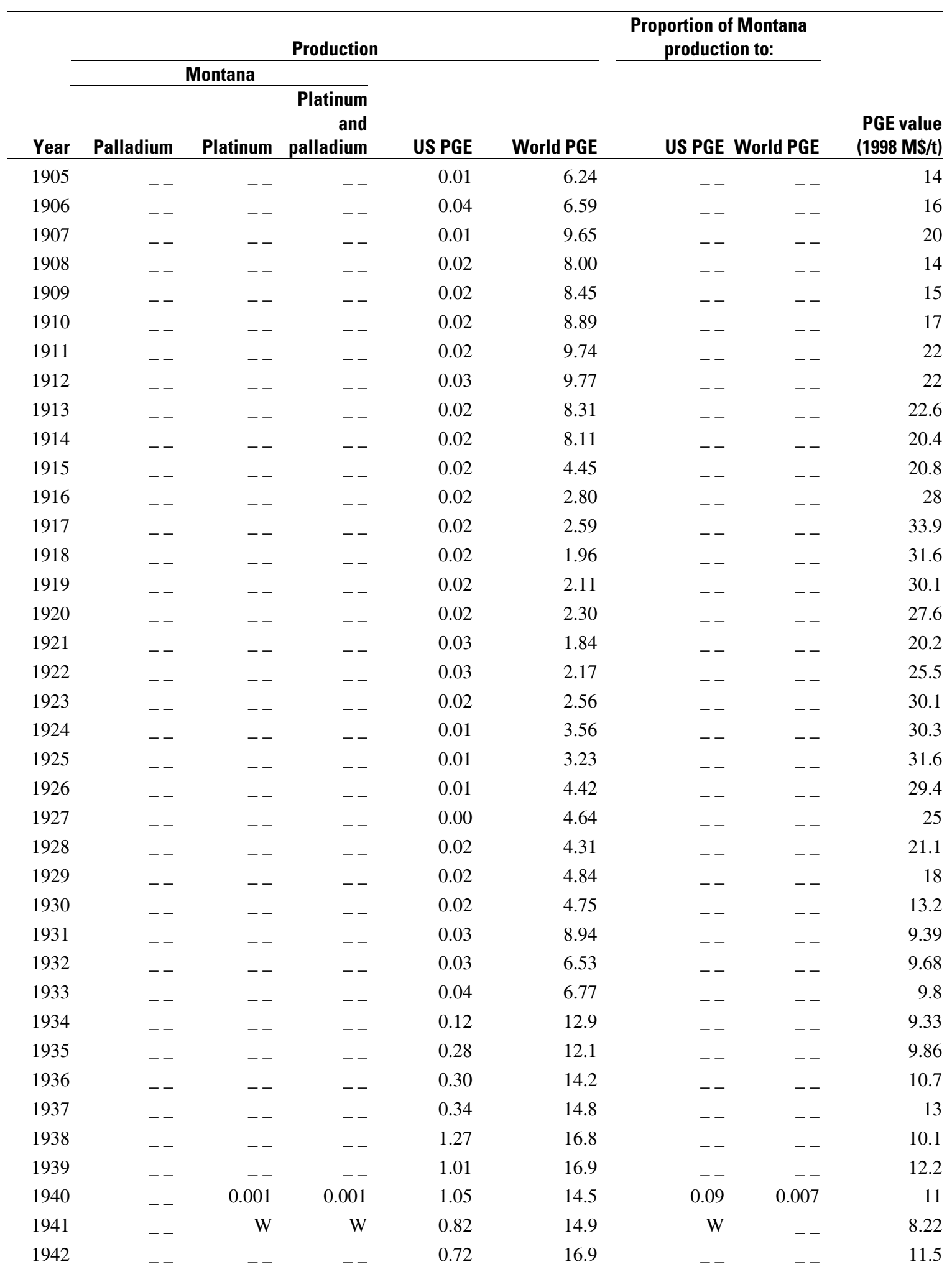




\begin{tabular}{|c|c|c|c|c|c|c|c|c|}
\hline 1943 & -- & -- & -- & 0.85 & 19.6 & -- & -- & 9.16 \\
\hline 1944 & -- & 0.0003 & 0.0003 & 1.05 & 16.0 & 0.03 & 0.002 & 8.93 \\
\hline 1945 & -- & -- & -- & 0.83 & 30.0 & -- & -_ & 8.83 \\
\hline 1946 & -- & -- & -- & 0.71 & 17.9 & -- & -- & 9.5 \\
\hline 1947 & -- & -- & - - & 0.43 & 15.6 & -- & -- & 8.98 \\
\hline 1948 & -- & -- & -- & 0.43 & 16.3 & -- & -- & 12 \\
\hline 1949 & -- & -- & -- & 0.77 & 17.9 & -- & -- & 12 \\
\hline 1950 & -- & -- & -- & 1.18 & 18.7 & -- & -- & 11.8 \\
\hline 1951 & - - & -- & - - & 1.15 & 21.0 & -- & -- & 12.1 \\
\hline 1952 & -- & -- & -- & 1.07 & 21.8 & -- & -- & 11.1 \\
\hline 1953 & -- & -- & -- & 0.81 & 24.1 & -- & -- & 12.2 \\
\hline 1954 & -- & -- & -- & 0.75 & 29.2 & -- & -- & 11.3 \\
\hline 1955 & -- & - - & -- & 0.72 & 33.9 & -- & -- & 9.33 \\
\hline 1956 & -- & -- & -- & 0.67 & 34.5 & -- & -- & 10.8 \\
\hline 1957 & -- & -- & -- & 0.58 & 41.1 & -- & -- & 9.83 \\
\hline 1958 & -- & -- & -- & 0.45 & 27.7 & -- & -- & 6.78 \\
\hline 1959 & - - & -_ & -- & 0.48 & 32.8 & -- & -- & 6.65 \\
\hline 1960 & -- & -- & -- & 0.73 & 39.7 & -- & -- & 8.85 \\
\hline 1961 & -- & -- & -- & 1.35 & 41.8 & -- & -- & 7.32 \\
\hline 1962 & -- & -- & -- & 0.89 & 50.5 & -- & -- & 7.89 \\
\hline 1963 & -- & -- & -- & 1.55 & 63.4 & -- & -- & 8.56 \\
\hline 1964 & -- & -- & -- & 1.26 & 79.2 & -- & -- & 9.68 \\
\hline 1965 & -- & -- & -- & 1.09 & 92.3 & -- & -- & 9.84 \\
\hline 1966 & -- & -- & -- & 1.60 & 94.5 & -- & -- & 9.95 \\
\hline 1967 & -- & -- & -- & 0.51 & 98.8 & -- & -- & 10.9 \\
\hline 1968 & -- & -- & -- & 0.46 & 106 & -- & -- & 10.7 \\
\hline 1969 & -- & -- & -- & 0.68 & 107 & -- & -- & 10.9 \\
\hline 1970 & -- & -- & -- & 0.54 & 132 & -- & -- & 10 \\
\hline 1971 & -- & -- & -- & 0.56 & 127 & -- & -- & 9.3 \\
\hline 1972 & -- & -- & -- & 0.53 & 133 & -- & -- & 9.83 \\
\hline 1973 & -- & -- & -- & 0.62 & 163 & -- & -- & 12.7 \\
\hline 1974 & -- & -- & -- & 0.39 & 179 & -- & -- & 16.5 \\
\hline 1975 & -- & -- & -- & 0.59 & 178 & -- & -- & 14.6 \\
\hline 1976 & -- & -- & -- & 0.19 & 194 & -- & -- & 10.1 \\
\hline 1977 & -- & -- & -- & 0.17 & 203 & -- & -- & 9.41 \\
\hline 1978 & -- & -- & -- & 0.26 & 200 & -- & -- & 12.4 \\
\hline 1979 & -- & -- & -- & 0.22 & 202 & -- & -- & 17.4 \\
\hline 1980 & -- & -- & -- & 0.09 & 213 & -- & -- & 21.4 \\
\hline 1981 & -- & -- & -- & 0.22 & 216 & -- & -- & 16.2 \\
\hline 1982 & -- & -- & -- & 0.25 & 200 & -- & -- & 12.1 \\
\hline 1983 & -- & -- & -- & 0.19 & 203 & -- & -- & 12.3 \\
\hline 1984 & -- & -- & -- & 0.47 & 238 & -- & -- & 12.6 \\
\hline 1985 & -- & -- & -- & -- & 247 & -- & -- & 12.5 \\
\hline 1986 & -- & -- & -- & -- & 260 & -- & -- & 14.4 \\
\hline 1987 & -- & W & W & 3.11 & 271 & W & W & 15.1 \\
\hline 1988 & -- & W & W & 4.97 & 280 & W & W & 14.9 \\
\hline 1989 & 4.85 & 1.43 & 6.28 & 6.28 & 282 & 100.00 & 2.23 & 16 \\
\hline 1990 & 5.93 & 1.81 & 7.74 & 7.74 & 291 & 100.00 & 2.66 & 19 \\
\hline
\end{tabular}




\begin{tabular}{rrrrrrrrr}
1991 & 6.05 & 1.73 & 7.78 & 7.78 & 287 & 100.00 & 2.71 & 16.6 \\
1992 & 6.47 & 1.84 & 8.31 & 7.74 & 280 & 107.36 & 2.97 & 13 \\
1993 & 6.5 & 1.8 & 8.3 & 8.83 & 276 & 94.00 & 3.01 & 9.66 \\
1994 & 6.44 & 1.96 & 8.4 & 8.40 & 269 & 100.00 & 3.12 & 9.16 \\
1995 & 5.26 & 1.59 & 6.85 & 6.85 & 326 & 100.00 & 2.10 & 7.7 \\
1996 & 6.1 & 1.84 & 7.94 & 7.94 & 324 & 100.00 & 2.45 & 8.72 \\
1997 & 8.4 & 2.61 & 11.01 & 11.0 & 333 & 100.00 & 3.31 & 8.59 \\
1998 & 10.6 & 3.22 & 13.82 & 13.8 & 348 & 100.00 & 3.97 & 10.2 \\
1999 & 9.8 & 2.92 & 12.72 & 12.7 & 376 & 100.00 & 3.38 & 10.3 \\
2000 & 10.3 & 3.11 & 13.41 & 13.4 & 365 & 100.00 & 3.67 & n. \\
2001 & 12 & 3.6 & 15.6 & n.d. & n.d. & n.d. & n.d. & n.d. \\
\hline
\end{tabular}




\section{Phosphate}

Phosphate rock was discovered in the Western Phosphate Field in 1889 and commercial production began in Idaho in 1906. Following a national trend, phosphate production in Idaho saw significant production increases during the late 1940s when open pit mining replaced underground mining, lowering production costs (Jasinsky and others, 2004). Idaho increased annual production from 125,000 metric tons in 1945 to 1,000,000 metric tons in the early 1950's. Idaho production continued to increase, reaching a maximum of 6,200,000 metric tons in 1999 (fig. 12A). Phosphate rock production in Montana began in 1929; however the transition to open pit mining was not feasible as in other states because the phosphate-bearing rocks were not amenable to open-pit methods. Phosphate rock mining in Montana eventually ceased in 1993 due to the high costs of underground mining (Jasinsky and others, 2004). U.S. phosphate production increased rapidly during the 1940s similar to the Idaho trends; however, this trend ended after 1980 (fig. 12D). The price of the commodity began to fall in 1976, and national production decreased (Kramer, undated). Nevertheless, production in Idaho remained strong, accounting for 8 to 14 percent of the U.S.'s phosphate rock production (fig. 12E) and nearly six percent of the world's production (fig. 12F). Despite the overall increased production in both the region and the U.S. over the course of the century, the average value of the commodity remained mostly constant at about $\$ 20$ to $\$ 40$ per ton, with the exception of anomalous spikes in about 1908 and again in 1976 (fig. 12G).

Table 27. Selected events affecting regional phosphate rock production

\begin{tabular}{lll}
\hline Year & Event & Reference \\
\hline 1889 & Discovery of Western Phosphate Field & Jasinsky and others, 2004 \\
1906 & Phosphate rock production begins in Idaho & Jasinsky and others, 2004 \\
1929 & Phosphate mining begins in Mont. & Jasinsky and others, 2004 \\
1940 s & Transition to open pit from underground mining & Jasinsky and others, 2004 \\
$1946-93$ & Gay mine in Idaho years of operation & Jasinsky and others, 2004 \\
1960 & Montana only remaining state using underground mining & Jasinsky and others, 2004 \\
1993 & Production ends in Montana due to high cost of underground & Jasinsky and others, 2004 \\
& mining & \\
\hline
\end{tabular}

Table 28. Selected events affecting U.S. phosphate rock production

\begin{tabular}{lll}
\hline Year & Event & Reference \\
\hline 1928 & Flotation first used to process phosphate ore & Kramer, undated \\
1979 & Phosphate mining ends in Wyoming & Kramer, undated \\
\hline
\end{tabular}



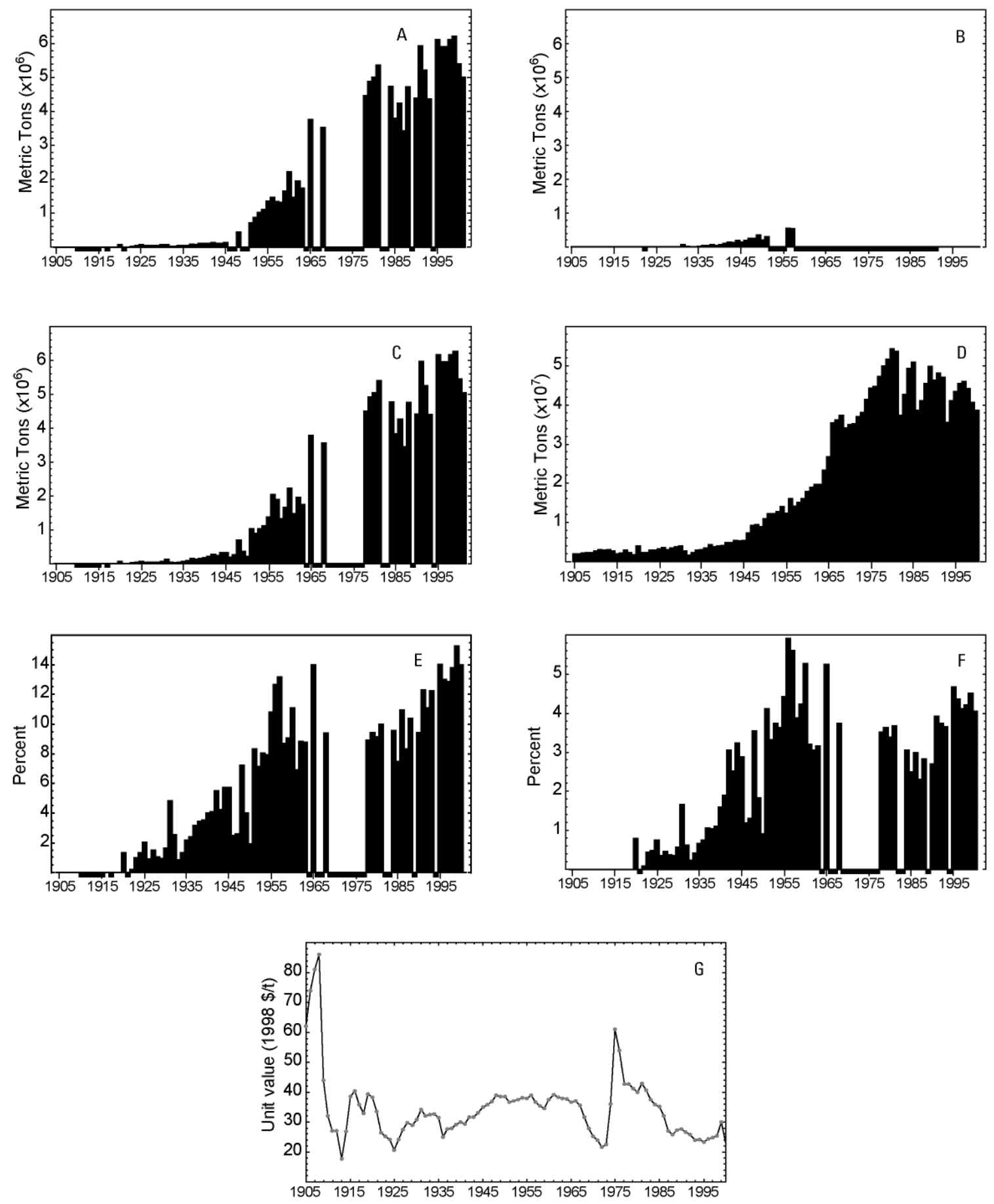

Figure 12. Phosphate rock production information from 1905-2001. State production data is shown in millions of metric tons and US production is shown in tens of millions of metric tons. A, annual production from Idaho. B, annual production from Montana. C, Idaho and Montana combined production. D, annual U.S. production. E, the proportion of regional phosphate rock production to U.S. production. $F$, the proportion of regional phosphate rock production to world production. $\mathrm{G}$, the historical value of phosphate rock in constant 1998 dollars. Bars extending below the $\mathrm{x}$-axis represent data withheld from publication. 
Table 29. Phosphate rock production from Idaho and Montana, the U.S., and the world, 1905-2001

[The table also summarizes the proportions of Idaho and Montana production relative to the US and the world as well as the price for phosphate rock in constant 1998 dollars. Data for state, US, and world production are given in metric tons; proportions of US and world production by Idaho and Montana are given in percent; phosphate rock value is in US dollars. W, withheld; n.d., no data; leaders ( $\ldots$ - ), no production]

\begin{tabular}{|c|c|c|c|c|c|c|c|c|}
\hline \multirow[b]{2}{*}{ Year } & \multicolumn{5}{|c|}{ Production } & \multicolumn{2}{|c|}{$\begin{array}{l}\text { Proportion of } \\
\text { Idaho and } \\
\text { Montana } \\
\text { production to: }\end{array}$} & \multirow[b]{2}{*}{$\begin{array}{r}\text { Phosphate } \\
\text { rock value } \\
(1998 \$ / t) \\
\end{array}$} \\
\hline & Idaho & Montana & $\begin{array}{r}\text { Idaho and } \\
\text { Montana } \\
\text { combined } \\
\end{array}$ & US & World & US & World & \\
\hline 1905 & -- & -- & - - & $1,980,000$ & $3,850,000$ & - - & - - & 62.00 \\
\hline 1906 & -- & -- & -- & $2,110,000$ & $4,190,000$ & -- & -- & 74.00 \\
\hline 1907 & -- & -- & -- & $2,300,000$ & $4,720,000$ & -- & -- & 81.00 \\
\hline 1908 & -- & - - & - - & $2,420,000$ & $5,380,000$ & -- & - - & 86.00 \\
\hline 1909 & -- & -- & -- & $2,380,000$ & $4,950,000$ & -- & -- & 44.00 \\
\hline 1910 & W & -- & W & $2,700,000$ & $5,430,000$ & W & -- & 32.00 \\
\hline 1911 & W & -- & W & $3,100,000$ & $5,940,000$ & W & -- & 27.00 \\
\hline 1912 & W & -- & $\mathrm{W}$ & $3,020,000$ & $6,730,000$ & W & -- & 27.00 \\
\hline 1913 & W & - - & W & $3,160,000$ & $7,230,000$ & W & - - & 17.80 \\
\hline 1914 & W & -- & $\mathrm{W}$ & $2,780,000$ & $5,420,000$ & W & -- & 26.80 \\
\hline 1915 & W & -- & W & $1,970,000$ & $4,120,000$ & W & -- & 38.50 \\
\hline 1916 & -- & -- & -- & $2,200,000$ & $4,830,000$ & -- & -- & 40.40 \\
\hline 1917 & W & -- & W & $2,900,000$ & $4,710,000$ & W & -- & 35.90 \\
\hline 1918 & -- & -- & -- & $2,320,000$ & $4,190,000$ & -- & -- & 33.00 \\
\hline 1919 & -- & -- & -- & $1,880,000$ & $4,150,000$ & - - & -- & 39.30 \\
\hline 1920 & 54,596 & -- & 54,596 & $4,040,000$ & $6,870,000$ & 1.31 & 0.79 & 38.30 \\
\hline 1921 & W & -- & - - & $2,470,000$ & $5,430,000$ & W & W & 33.50 \\
\hline 1922 & 4,553 & W & 4,553 & $2,370,000$ & $5,940,000$ & 0.19 & 0.08 & 26.50 \\
\hline 1923 & 30,568 & - - & 30,568 & $2,990,000$ & $7,120,000$ & 0.99 & 0.43 & 25.20 \\
\hline 1924 & 37,420 & 499 & 37,919 & $2,890,000$ & $7,780,000$ & 1.31 & 0.49 & 24.10 \\
\hline 1925 & 66,992 & -- & 67,491 & $3,310,000$ & $8,900,000$ & 2.04 & 0.76 & 20.70 \\
\hline 1926 & 33,644 & -- & 33,644 & $3,650,000$ & $9,380,000$ & 0.92 & 0.36 & 24.20 \\
\hline 1927 & 45,986 & -- & 45,986 & $3,070,000$ & $9,990,000$ & 1.50 & 0.46 & 27.50 \\
\hline 1928 & 38,078 & -- & 38,078 & $3,580,000$ & $10,100,000$ & 1.06 & 0.38 & 29.80 \\
\hline 1929 & 36,475 & 41 & 36,516 & $3,880,000$ & $10,400,000$ & 0.94 & 0.35 & 29.00 \\
\hline 1930 & 60,894 & 6,101 & 66,995 & $4,020,000$ & $11,800,000$ & 1.67 & 0.57 & 30.90 \\
\hline 1931 & 61,957 & 68,982 & 130,939 & $2,710,000$ & $7,860,000$ & 4.83 & 1.67 & 34.20 \\
\hline 1932 & 23,544 & 20,412 & 43,956 & $1,730,000$ & $7,110,000$ & 2.54 & 0.62 & 32.10 \\
\hline 1933 & 20,068 & 500 & 20,568 & $2,400,000$ & $8,900,000$ & 0.86 & 0.23 & 32.60 \\
\hline 1934 & 37,747 & 2,119 & 39,867 & $2,950,000$ & $9,510,000$ & 1.35 & 0.42 & 32.70 \\
\hline 1935 & 42,467 & 27,938 & 70,405 & $3,210,000$ & $10,500,000$ & 2.19 & 0.67 & 31.40 \\
\hline 1936 & 47,869 & 36,600 & 84,469 & $3,520,000$ & $11,300,000$ & 2.40 & 0.75 & 25.10 \\
\hline 1937 & 84,775 & 51,650 & 136,425 & $4,330,000$ & $12,900,000$ & 3.15 & 1.06 & 27.70 \\
\hline 1938 & 67,073 & 67,558 & 134,631 & $3,920,000$ & $12,900,000$ & 3.43 & 1.04 & 28.00 \\
\hline 1939 & 96,983 & 45,096 & 142,079 & $4,050,000$ & $12,800,000$ & 3.51 & 1.11 & 29.10 \\
\hline 1940 & 100,678 & 65,270 & 165,948 & $4,130,000$ & $10,300,000$ & 4.02 & 1.61 & 29.90 \\
\hline 1941 & 98,835 & 106,795 & 205,630 & $5,000,000$ & $10,800,000$ & 4.11 & 1.90 & 29.40 \\
\hline 1942 & 115,910 & 152,815 & 268,725 & $4,900,000$ & $8,800,000$ & 5.48 & 3.05 & 31.70 \\
\hline 1943 & 110,664 & 121,686 & 232,350 & $5,460,000$ & $9,250,000$ & 4.26 & 2.51 & 31.70 \\
\hline
\end{tabular}




\begin{tabular}{|c|c|c|c|c|c|c|c|c|}
\hline 1944 & 114,371 & 189,426 & 303,797 & $5,280,000$ & $9,330,000$ & 5.75 & 3.26 & 33.10 \\
\hline 1945 & 125,319 & 153,279 & 278,598 & $5,490,000$ & $10,900,000$ & 5.07 & 2.56 & 35.00 \\
\hline 1946 & $\mathrm{~W}$ & 182,832 & 182,832 & $7,280,000$ & $15,300,000$ & 2.51 & 1.19 & 35.80 \\
\hline 1947 & W & 240,020 & 240,020 & $9,260,000$ & $18,300,000$ & 2.59 & 1.31 & 36.80 \\
\hline 1948 & 437,916 & 252,996 & 690,912 & $9,540,000$ & $19,400,000$ & 7.24 & 3.56 & 38.90 \\
\hline 1949 & W & 360,697 & 360,697 & $9,020,000$ & $19,700,000$ & 4.00 & 1.83 & 38.60 \\
\hline 1950 & W & 213,370 & 213,370 & $10,900,000$ & $23,400,000$ & 1.96 & 0.91 & 38.60 \\
\hline 1951 & 704,250 & 308,878 & $1,013,128$ & $12,200,000$ & $24,600,000$ & 8.30 & 4.12 & 36.70 \\
\hline 1952 & 880,232 & W & 880,232 & $12,300,000$ & $26,400,000$ & 7.16 & 3.33 & 37.10 \\
\hline 1953 & $1,018,047$ & W & $1,018,047$ & $12,700,000$ & $27,200,000$ & 8.02 & 3.74 & 37.40 \\
\hline 1954 & $1,110,539$ & W & $1,110,539$ & $14,000,000$ & $30,500,000$ & 7.93 & 3.64 & 38.10 \\
\hline 1955 & $1,351,342$ & W & $1,351,342$ & $12,500,000$ & $30,500,000$ & 10.81 & 4.43 & 38.00 \\
\hline 1956 & $1,461,075$ & 566,954 & $2,028,030$ & $16,000,000$ & $34,200,000$ & 12.68 & 5.93 & 38.90 \\
\hline 1957 & $1,327,973$ & 542,569 & $1,870,542$ & $14,200,000$ & $33,200,000$ & 13.17 & 5.63 & 36.60 \\
\hline 1958 & $1,311,717$ & W & $1,311,717$ & $15,100,000$ & $33,700,000$ & 8.69 & 3.89 & 35.50 \\
\hline 1959 & $1,635,836$ & W & $1,635,836$ & $16,100,000$ & $38,400,000$ & 10.16 & 4.26 & 34.50 \\
\hline 1960 & $2,211,934$ & W & $2,211,934$ & $17,800,000$ & $41,800,000$ & 12.43 & 5.29 & 37.50 \\
\hline 1961 & $1,463,108$ & W & $1,463,108$ & $18,900,000$ & $45,500,000$ & 7.74 & 3.22 & 39.10 \\
\hline 1962 & $1,942,682$ & W & $1,942,682$ & $19,700,000$ & $63,300,000$ & 9.86 & 3.07 & 38.40 \\
\hline 1963 & $1,727,309$ & W & $1,727,309$ & $19,700,000$ & $54,600,000$ & 8.77 & 3.16 & 38.00 \\
\hline 1964 & W & $\mathrm{W}$ & W & $23,300,000$ & $63,700,000$ & W & W & 37.70 \\
\hline 1965 & $3,759,375$ & $\mathrm{~W}$ & $3,759,375$ & $26,900,000$ & $71,400,000$ & 13.98 & 5.27 & 36.70 \\
\hline 1966 & W & W & W & $35,400,000$ & $84,500,000$ & W & W & 37.10 \\
\hline 1967 & W & W & W & $36,100,000$ & $87,300,000$ & W & W & 35.70 \\
\hline 1968 & $3,519,029$ & W & $3,519,029$ & $37,400,000$ & $94,100,000$ & 9.41 & 3.74 & 31.70 \\
\hline 1969 & W & $\mathrm{W}$ & W & $34,200,000$ & $92,100,000$ & W & W & 27.90 \\
\hline 1970 & W & W & $\mathrm{W}$ & $35,100,000$ & $95,100,000$ & W & W & 25.20 \\
\hline 1971 & W & W & W & $35,200,000$ & $94,000,000$ & W & W & 23.90 \\
\hline 1972 & W & W & $\mathrm{W}$ & $37,000,000$ & $101,000,000$ & W & W & 21.70 \\
\hline 1973 & W & W & $\mathrm{W}$ & $38,200,000$ & $111,000,000$ & $\mathrm{~W}$ & W & 22.40 \\
\hline 1974 & $\mathrm{~W}$ & $\mathrm{~W}$ & $\mathrm{~W}$ & $41,400,000$ & $123,000,000$ & $\mathrm{~W}$ & $\mathrm{~W}$ & 36.13 \\
\hline 1975 & W & W & W & $44,300,000$ & $109,000,000$ & W & W & 61.07 \\
\hline 1976 & W & W & W & $44,700,000$ & $109,000,000$ & W & W & 53.97 \\
\hline 1977 & W & W & W & $47,300,000$ & $121,000,000$ & W & W & 42.76 \\
\hline 1978 & $4,461,000$ & W & $4,461,000$ & $50,000,000$ & $127,000,000$ & 8.92 & 3.51 & 42.68 \\
\hline 1979 & $4,880,000$ & W & $4,880,000$ & $51,600,000$ & $134,000,000$ & 9.46 & 3.64 & 41.31 \\
\hline 1980 & $4,991,000$ & W & $4,991,000$ & $54,400,000$ & $147,000,000$ & 9.17 & 3.40 & 40.00 \\
\hline 1981 & $5,361,000$ & W & $5,361,000$ & $53,600,000$ & $145,000,000$ & 10.00 & 3.70 & 42.84 \\
\hline 1982 & W & $\mathrm{W}$ & W & $37,400,000$ & $129,000,000$ & W & W & 40.59 \\
\hline 1983 & $\mathrm{~W}$ & $\mathrm{~W}$ & $\mathrm{~W}$ & $42,600,000$ & $143,000,000$ & $\mathrm{~W}$ & $\mathrm{~W}$ & 37.40 \\
\hline 1984 & $4,722,000$ & $\mathrm{~W}$ & $4,722,000$ & $49,200,000$ & $154,000,000$ & 9.60 & 3.07 & 35.83 \\
\hline 1985 & $3,784,000$ & W & $3,784,000$ & $50,800,000$ & $151,000,000$ & 7.45 & 2.51 & 35.19 \\
\hline 1986 & $4,235,000$ & W & $4,235,000$ & $38,700,000$ & $141,000,000$ & 10.94 & 3.00 & 32.08 \\
\hline 1987 & $3,411,000$ & W & $3,411,000$ & $41,000,000$ & $147,000,000$ & 8.32 & 2.32 & 27.08 \\
\hline 1988 & $4,706,000$ & $\mathrm{~W}$ & $4,706,000$ & $45,400,000$ & $166,000,000$ & 10.37 & 2.83 & 25.76 \\
\hline 1989 & W & W & W & $49,800,000$ & $163,000,000$ & W & W & 27.29 \\
\hline 1990 & $4,380,000$ & W & $4,380,000$ & $46,300,000$ & $162,000,000$ & 9.46 & 2.70 & 27.80 \\
\hline 1991 & $5,921,000$ & $\mathrm{~W}$ & $5,921,000$ & $48,100,000$ & $150,000,000$ & 12.31 & 3.95 & 26.66 \\
\hline 1992 & $5,208,000$ & - - & $5,208,000$ & $47,000,000$ & $139,000,000$ & 11.08 & 3.75 & 25.89 \\
\hline 1993 & $4,355,000$ & -- & $4,355,000$ & $35,500,000$ & $119,000,000$ & 12.27 & 3.66 & 24.02 \\
\hline
\end{tabular}




\begin{tabular}{rrrrrrrrr}
1994 & W & -- & W & $41,100,000$ & $127,000,000$ & W & W & 24.23 \\
1995 & $6,100,000$ & -- & $6,100,000$ & $43,500,000$ & $130,000,000$ & 14.02 & 4.69 & 23.26 \\
1996 & $5,900,000$ & -- & $5,900,000$ & $45,400,000$ & $135,000,000$ & 13.00 & 4.37 & 24.31 \\
1997 & $5,900,000$ & -- & $5,900,000$ & $45,900,000$ & $143,000,000$ & 12.85 & 4.13 & 24.78 \\
1998 & $6,100,000$ & -- & $6,100,000$ & $44,200,000$ & $144,000,000$ & 13.80 & 4.24 & 25.46 \\
1999 & $6,200,000$ & -- & $6,200,000$ & $40,600,000$ & $137,000,000$ & 15.27 & 4.53 & 29.90 \\
2000 & $5,400,000$ & -- & $5,400,000$ & $38,600,000$ & $133,000,000$ & 13.99 & 4.06 & 22.86 \\
2001 & $5,000,000$ & -- & $5,000,000$ & n.d. & n.d. & n.d. & n.d. & n.d. \\
\hline
\end{tabular}




\section{Pumice}

Montana is not a significant producer of pumice; therefore no data regarding production from that state are included in this report. Pumice production in Idaho began in 1945, but annual production has varied considerably from year to year (fig. 13A). Idaho pumice production has ranged from less than 20,000 metric tons in 1969 to as much as 159,000 tons in 1996, although the production information was withheld for the period of 1976-89. U.S. production increased substantially after 1945 and has similarly varied drastically by year (fig. 13B). Idaho accounted for nearly 35 percent of the national pumice production in 1946, while the annual production in 1968 and 1996 was over 25 percent of national production (fig. 13C). Idaho generally averages 5 to 15 percent of the nation's production of pumice and 0.25 to 1 percent of the world's production (fig. 13D). However, the figures for world pumice production include abundant resources in the form of volcanic cinders, which are not included in the production from Idaho. This difference in the method of pumice production reporting tends to marginalize U.S. production when compared to global production. For the last 50 years, the value of pumice has ranged between $\$ 10$ and $\$ 30$ per ton. Values had been as high as $\$ 80$ per ton prior to pumice production in Idaho (fig. 13E).
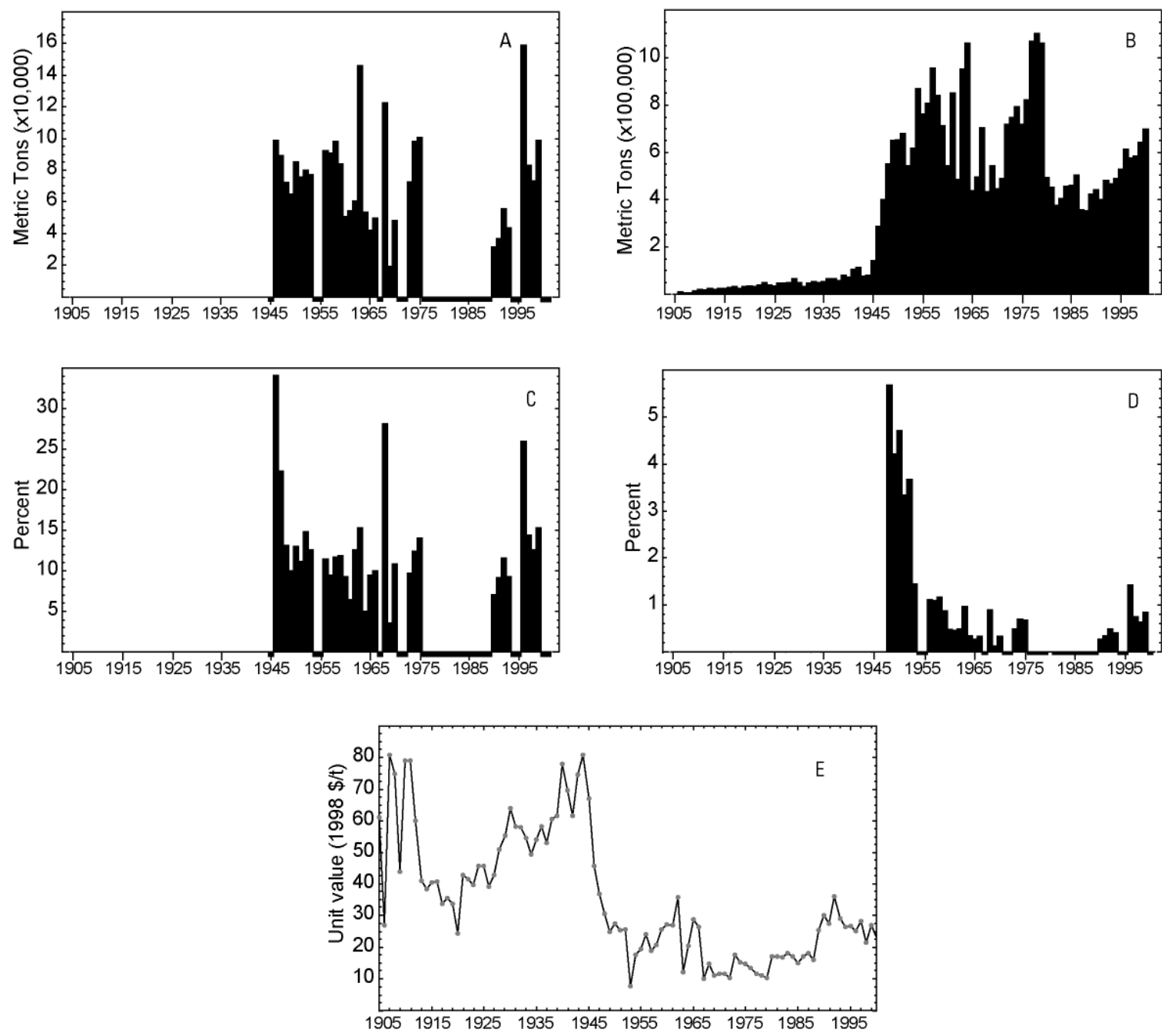

Figure 13. Pumice production information from 1905-2001. A, annual production from Idaho. B, annual U.S. production. C, the proportion of Idaho pumice production to U.S. production. D, the proportion of Idaho pumice production to world production. E, the historical value of pumice in constant 1998 dollars. Bars extending below the x-axis represent data withheld from publication. 
Table 30. Pumice production from Idaho, the U.S., and the world, 1905-2001

[The table also summarizes the proportions of Idaho production relative to the US and the world as well as the price for pumice in constant 1998 dollars. World pumice production data includes volcanic cinders. Data for state, US, and world production are given in metric tons; proportions of US and world production by Idaho are given in percent; pumice value is in US dollars. W, withheld; n.d., no data; leaders ( $\_$- $)$, no production]

\begin{tabular}{|c|c|c|c|c|c|c|}
\hline \multirow[b]{2}{*}{ Year } & \multicolumn{3}{|c|}{ Production } & \multicolumn{2}{|c|}{ Proportion of Idaho production to: } & \multirow{2}{*}{$\begin{array}{r}\text { Pumice value } \\
(1998 \$ / t)\end{array}$} \\
\hline & Idaho & US & World & US & World & \\
\hline 1905 & -_ & 1,660 & n.d. & $\ldots$ & n.d. & 61.00 \\
\hline 1906 & - - & 11,100 & n.d. & _- & n.d. & 27.00 \\
\hline 1907 & -- & 7,360 & n.d. & -- & n.d. & 81.00 \\
\hline 1908 & -- & 9,590 & n.d. & -- & n.d. & 75.00 \\
\hline 1909 & -- & 13,700 & n.d. & -- & n.d. & 44.00 \\
\hline 1910 & -- & 21,100 & n.d. & -- & n.d. & 79.00 \\
\hline 1911 & -- & 19,700 & n.d. & -- & n.d. & 79.00 \\
\hline 1912 & -- & 24,600 & n.d. & -- & n.d. & 60.00 \\
\hline 1913 & -- & 22,300 & n.d. & -- & n.d. & 41.00 \\
\hline 1914 & -- & 25,000 & n.d. & -- & n.d. & 38.50 \\
\hline 1915 & -- & 25,100 & n.d. & -- & n.d. & 40.50 \\
\hline 1916 & -- & 30,200 & n.d. & -- & n.d. & 40.70 \\
\hline 1917 & -- & 32,000 & n.d. & -- & n.d. & 33.80 \\
\hline 1918 & -- & 27,800 & n.d. & -- & n.d. & 35.50 \\
\hline 1919 & -- & 32,700 & n.d. & -- & n.d. & 33.70 \\
\hline 1920 & -- & 38,000 & 66,000 & -- & -- & 24.50 \\
\hline 1921 & -- & 33,700 & 47,600 & -- & -- & 42.80 \\
\hline 1922 & -- & 41,100 & 240,000 & -- & -- & 41.60 \\
\hline 1923 & -- & 51,300 & 275,000 & -- & -- & 39.80 \\
\hline 1924 & -- & 39,600 & 157,000 & -- & -- & 45.80 \\
\hline 1925 & -- & 36,600 & 166,000 & -- & -- & 45.60 \\
\hline 1926 & -- & 48,900 & n.d. & -- & n.d. & 39.20 \\
\hline 1927 & -- & 48,400 & n.d. & -- & n.d. & 42.90 \\
\hline 1928 & -- & 52,100 & n.d. & -- & n.d. & 51.00 \\
\hline 1929 & -- & 67,000 & n.d. & -- & n.d. & 55.30 \\
\hline 1930 & -- & 51,600 & n.d. & -- & n.d. & 63.90 \\
\hline 1931 & -- & 35,000 & n.d. & -- & n.d. & 58.10 \\
\hline 1932 & -- & 48,300 & n.d. & -- & n.d. & 58.00 \\
\hline 1933 & -- & 55,500 & n.d. & -- & n.d. & 54.50 \\
\hline 1934 & -- & 50,900 & n.d. & -- & n.d. & 49.50 \\
\hline 1935 & -- & 54,400 & n.d. & -- & n.d. & 54.10 \\
\hline 1936 & -- & 66,100 & n.d. & -- & n.d. & 58.20 \\
\hline 1937 & -- & 64,400 & n.d. & -- & n.d. & 53.10 \\
\hline 1938 & -- & 59,600 & n.d. & -- & n.d. & 60.70 \\
\hline 1939 & -- & 80,900 & n.d. & -- & n.d. & 61.60 \\
\hline 1940 & -- & 74,800 & n.d. & -- & n.d. & 77.90 \\
\hline 1941 & -- & 106,000 & n.d. & -- & n.d. & 69.70 \\
\hline 1942 & -- & 115,000 & n.d. & -- & n.d. & 61.50 \\
\hline 1943 & -- & 77,200 & n.d. & -- & n.d. & 74.70 \\
\hline
\end{tabular}




\begin{tabular}{|c|c|c|c|c|c|c|}
\hline 1944 & -- & 80,500 & n.d. & -- & n.d. & 80.90 \\
\hline 1945 & W & 142,000 & n.d. & $\mathrm{W}$ & n.d. & 67.00 \\
\hline 1946 & 98,746 & 290,000 & n.d. & 34.050 & n.d. & 45.60 \\
\hline 1947 & 89,466 & 401,000 & n.d. & 22.311 & n.d. & 36.80 \\
\hline 1948 & 72,055 & 551,000 & $1,270,000$ & 13.077 & 5.6736431 & 30.70 \\
\hline 1949 & 64,750 & 650,000 & $1,540,000$ & 9.961 & 4.2045185 & 25.00 \\
\hline 1950 & 85,268 & 653,000 & $1,810,000$ & 13.058 & 4.7109242 & 27.60 \\
\hline 1951 & 75,777 & 680,000 & $2,270,000$ & 11.144 & 3.3381763 & 25.30 \\
\hline 1952 & 79,911 & 542,000 & $2,180,000$ & 14.744 & 3.665629 & 25.60 \\
\hline 1953 & 77,315 & 615,000 & $5,350,000$ & 12.572 & 1.4451442 & 7.74 \\
\hline 1954 & W & 868,000 & $5,620,000$ & W & W & 17.50 \\
\hline 1955 & W & 764,000 & $6,530,000$ & W & W & 19.50 \\
\hline 1956 & 92,534 & 805,000 & $8,350,000$ & 11.49 & 1.11 & 24.00 \\
\hline 1957 & 90,720 & 957,000 & $8,260,000$ & 9.48 & 1.10 & 18.80 \\
\hline 1958 & 97,978 & 839,000 & $8,440,000$ & 11.68 & 1.16 & 20.80 \\
\hline 1959 & 84,370 & 711,000 & $9,710,000$ & 11.87 & 0.87 & 25.70 \\
\hline 1960 & 50,803 & 545,000 & $10,800,000$ & 9.32 & 0.47 & 27.30 \\
\hline 1961 & 54,432 & 849,000 & $11,900,000$ & 6.41 & 0.46 & 27.10 \\
\hline 1962 & 60,782 & 484,000 & $12,400,000$ & 12.56 & 0.49 & 35.90 \\
\hline 1963 & 146,059 & 953,000 & $15,100,000$ & 15.33 & 0.97 & 12.20 \\
\hline 1964 & 53,525 & $1,060,000$ & $15,200,000$ & 5.05 & 0.35 & 20.40 \\
\hline 1965 & 41,731 & 438,000 & $14,900,000$ & 9.53 & 0.28 & 28.90 \\
\hline 1966 & 49,896 & 498,000 & $14,700,000$ & 10.02 & 0.34 & 26.50 \\
\hline 1967 & W & 704,000 & $14,000,000$ & W & W & 10.00 \\
\hline 1968 & 122,472 & 436,000 & $13,600,000$ & 28.09 & 0.90 & 14.70 \\
\hline 1969 & 19,051 & 543,000 & $14,700,000$ & 3.51 & 0.13 & 11.06 \\
\hline 1970 & 48,082 & 445,000 & $14,300,000$ & 10.80 & 0.34 & 11.68 \\
\hline 1971 & W & 490,000 & $14,800,000$ & $\mathrm{~W}$ & W & 11.51 \\
\hline 1972 & W & 717,000 & $15,600,000$ & W & W & 10.22 \\
\hline 1973 & 72,576 & 748,000 & $14,900,000$ & 9.70 & 0.49 & 17.73 \\
\hline 1974 & 97,978 & 792,000 & $14,000,000$ & 12.37 & 0.70 & 15.31 \\
\hline 1975 & 100,699 & 717,000 & $14,700,000$ & 14.04 & 0.69 & 14.75 \\
\hline 1976 & $\mathrm{~W}$ & 822,000 & $15,800,000$ & $\mathrm{~W}$ & W & 13.35 \\
\hline 1977 & W & $1,070,000$ & $15,500,000$ & W & W & 11.65 \\
\hline 1978 & W & $1,100,000$ & $17,800,000$ & W & W & 11.03 \\
\hline 1979 & W & $1,060,000$ & $17,700,000$ & W & W & 10.28 \\
\hline 1980 & W & 493,000 & n.d. & W & n.d. & 17.13 \\
\hline 1981 & $\mathrm{~W}$ & 453,000 & $12,800,000$ & W & $\mathrm{W}$ & 17.07 \\
\hline 1982 & W & 377,000 & $11,700,000$ & W & W & 16.77 \\
\hline 1983 & W & 407,000 & $10,700,000$ & W & W & 18.02 \\
\hline 1984 & W & 455,000 & $12,200,000$ & W & W & 16.98 \\
\hline 1985 & W & 461,000 & $11,000,000$ & W & W & 14.97 \\
\hline 1986 & W & 503,000 & $10,400,000$ & W & W & 17.01 \\
\hline 1987 & W & 356,000 & $10,700,000$ & W & W & 18.11 \\
\hline 1988 & W & 353,000 & $10,800,000$ & W & W & 16.12 \\
\hline 1989 & W & 424,000 & $12,300,000$ & W & W & 25.48 \\
\hline 1990 & 31,333 & 443,000 & $11,000,000$ & 7.07 & 0.28 & 30.09 \\
\hline 1991 & 36,868 & 401,000 & $10,800,000$ & 9.19 & 0.34 & 27.41 \\
\hline
\end{tabular}




\begin{tabular}{rrrrrrr}
1992 & 55,525 & 481,000 & $11,100,000$ & 11.54 & 0.50 & 36.01 \\
1993 & 43,438 & 469,000 & $11,000,000$ & 9.26 & 0.39 & 28.97 \\
1994 & $\mathrm{~W}$ & 490,000 & $11,400,000$ & $\mathrm{~W}$ & $\mathrm{~W}$ & 26.51 \\
1995 & $\mathrm{~W}$ & 529,000 & $10,800,000$ & $\mathrm{~W}$ & $\mathrm{~W}$ & 26.74 \\
1996 & 159,000 & 612,000 & $11,100,000$ & 25.98 & 1.43 & 25.13 \\
1997 & 83,100 & 577,000 & $11,200,000$ & 14.40 & 0.74 & 28.33 \\
1998 & 73,400 & 583,000 & $11,500,000$ & 12.59 & 0.64 & 21.59 \\
1999 & 98,600 & 643,000 & $11,600,000$ & 15.33 & 0.85 & 27.09 \\
2000 & $\mathrm{~W}$ & 697,000 & $12,000,000$ & $\mathrm{~W}$ & $\mathrm{~W}$ & 22.98 \\
2001 & $\mathrm{~W}$ & n.d. & n.d. & $\mathrm{W}$ & n.d. & n.d. \\
\hline
\end{tabular}




\section{Silver}

Numerous silver mines were developed throughout Idaho in the latter half of the $19^{\text {th }}$ century; however, in 1934-35 the state saw a substantial increase in silver production when the Idaho Silver Belt in the Coeur d'Alene district began major production (Heyl and others, 1973). High-grade ore from the Sunshine mine contributed to the largest production year for Idaho in 1937 with over 600 metric tons. Since then, Idaho has consistently been an important producer of silver, often producing well over 400 metric tons per year. Montana was a significant silver producer as well in the early $20^{\text {th }}$ century; however by the 1970's, Montana's silver industry decreased to about 100 metric tons per year (fig. 14B). This declining trend reversed in 1982 when the Troy mine began operation, but by 2000, three of Montana's top silver producing mines, the Troy, Zortman, and Continental mines had all closed or suspended operations. Combined, Idaho and Montana consistently produced more than 500 metric tons of silver, though recent production rates for the region have dropped below 400 metric tons per year (fig. 14C). In contrast, US silver production has risen in recent years to more than 2000 metric tons per year (fig. 14D). In 1960, silver mining in Idaho and Montana was responsible for more than 60 percent of the nation's production, although by 1998 the proportion had decreased to about 20 percent (fig. 14E). Idaho and Montana accounted for 17 percent of the world's silver production during World War I, but their contribution dropped below 3 percent by 1998 (fig. 14F; Heyl and others, 1973). Production data for Montana are withheld after 1996.

Table 31. Selected events affecting regional silver production

\begin{tabular}{lll}
\hline Year & Event & Reference \\
\hline 1921 & Ore found on Yankee Boy vein by Sunshine Mining Co., builds small mill & this study \\
Apr. 1921 - & Low copper price closes Butte mines & Everett, 2002 \\
Jan. 1922 & & \\
1930 & Severe curtailment of mining operations due to collapse of metals prices & this study \\
1939 & Mineral Point mine developed, expanding Silver Belt production & this study \\
1945 & Production decreases due to wartime labor shortage & this study \\
1952 & Galena mine development begins & this study \\
1956 & Sunshine mine strike & Kiilsgaard, 1964 \\
July 1967 - & Longest strike in Butte's history - 8 1/2 months & Everett, 2002 \\
Mar. 1968 & & \\
May 1972 & Fire at Sunshine mine kills 91 men and closes mine for 7 months & Heyl and others, 1973 \\
1976 & Couer mine placed in production & this study \\
$1982-1993$ & Montana's Troy mine years of operation & this study \\
$1979-1998$ & Zortman-Landusky mine in Mont. Years of operation & this study \\
2000 & Continental mine in Mont. suspends operation & this study \\
2001 & Sunshine mine in Idaho closed & this study \\
\hline
\end{tabular}

Table 32. Selected events affecting U.S. silver production

\begin{tabular}{lll}
\hline Year & Event & Reference \\
\hline$\sim 1915$ & Peak production owing to coinage demand of WWI allies & Heyl and others, 1973 \\
1918 & $\begin{array}{l}\text { Pittman Act to convert silver dollars to bullion } \\
\text { Technological advances during the war in electronics and } \\
\text { photography lead to increased consumer demand after WWII }\end{array}$ & Hilliard, 1999b \\
WWII era & Production begins at Viburnum Trend & Kramer, undated \\
1960 & Silver Purchase Act repealed & Hilliard, 1999b \\
1963 & Coinage act of 1965 eliminates silver from most US coins & this study \\
1965 & Price of silver sets record high & Kramer, undated \\
1980 & US mint began minting silver bullion coin & Hilliard, 1999b \\
\hline
\end{tabular}



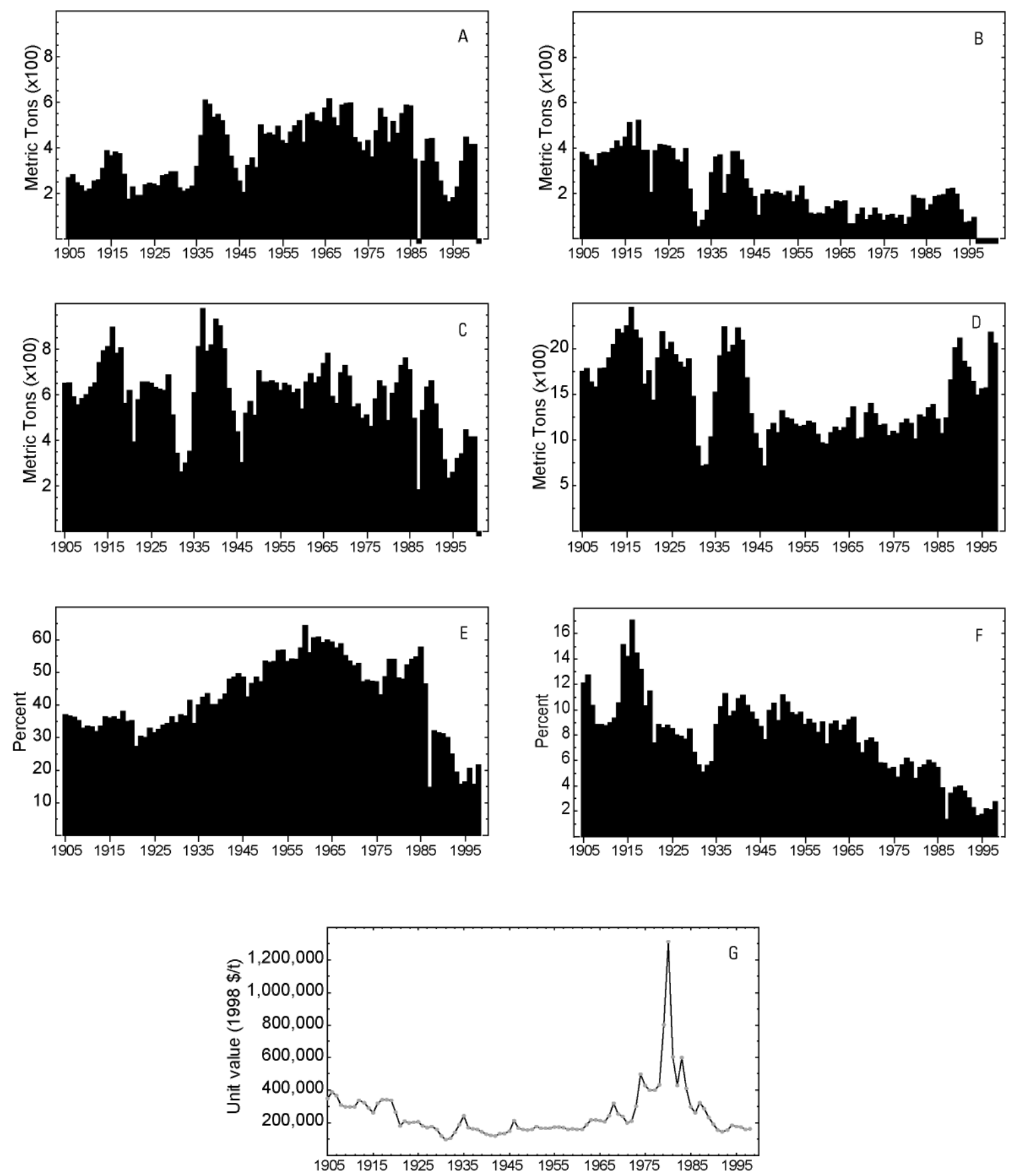

Figure 14. Silver production information from 1905-2001. A, annual production from Idaho. B, annual production from Montana. C, Idaho and Montana combined production. D, annual U.S. domestic mine production of silver. E, the proportion of regional silver production to U.S. production. $F$, the proportion of regional silver production to world production. $G$, the historical value of silver in constant 1998 dollars. Bars extending below the $x$-axis represent data withheld from publication. 
Table 33. Silver production from Idaho and Montana, the U.S., and the world, 1905-2001

[The table also summarizes the proportions of Idaho and Montana production relative to the US and the world as well as the price for silver in constant 1998 dollars. Data for state, US, and world production are given in metric tons; proportions of US and world production by Idaho and Montana are given in percent; silver value is in US dollars. W, withheld; n.d., no data]

\begin{tabular}{|c|c|c|c|c|c|c|c|c|}
\hline \multirow[b]{2}{*}{ Year } & \multicolumn{5}{|c|}{ Production } & \multicolumn{2}{|c|}{$\begin{array}{l}\text { Proportion of Idaho and } \\
\text { Montana production to: }\end{array}$} & \multirow[b]{2}{*}{$\begin{array}{r}\text { Silver value } \\
(1998 \$ / \mathbf{t}) \\
\end{array}$} \\
\hline & Idaho & Montana & $\begin{array}{l}\text { Idaho and } \\
\text { Montana } \\
\text { combined }\end{array}$ & US & World & US & World & \\
\hline 1905 & 270 & 380 & 650 & 1,750 & 5,360 & 37.16 & 12.13 & 350,000 \\
\hline 1906 & 281 & 373 & 653 & 1,780 & 5,130 & 36.69 & 12.73 & 390,000 \\
\hline 1907 & 245 & 346 & 592 & 1,630 & 5,730 & 36.29 & 10.32 & 370,000 \\
\hline 1908 & 235 & 322 & 557 & 1,580 & 6,320 & 35.27 & 8.82 & 310,000 \\
\hline 1909 & 210 & 374 & 584 & 1,780 & 6,600 & 32.83 & 8.86 & 300,000 \\
\hline 1910 & 219 & 382 & 601 & 1,790 & 6,900 & 33.55 & 8.70 & 300,000 \\
\hline 1911 & 255 & 378 & 633 & 1,900 & 7,040 & 33.31 & 8.99 & 300,000 \\
\hline 1912 & 258 & 396 & 654 & 2,050 & 6,980 & 31.90 & 9.37 & 340,000 \\
\hline 1913 & 311 & 430 & 741 & 2,210 & 7,010 & 33.51 & 10.56 & 323,000 \\
\hline 1914 & 388 & 405 & 793 & 2,170 & 5,240 & 36.54 & 15.13 & 292,000 \\
\hline 1915 & 366 & 447 & 813 & 2,250 & 5,730 & 36.15 & 14.19 & 263,000 \\
\hline 1916 & 383 & 513 & 896 & 2,450 & 5,250 & 36.56 & 17.06 & 322,000 \\
\hline 1917 & 374 & 408 & 782 & 2,200 & 5,420 & 35.57 & 14.44 & 344,000 \\
\hline 1918 & 285 & 522 & 808 & 2,120 & 6,140 & 38.10 & 13.16 & 341,000 \\
\hline 1919 & 174 & 390 & 564 & 1,610 & 5,490 & 35.01 & 10.27 & 339,000 \\
\hline 1920 & 228 & 391 & 619 & 1,760 & 5,390 & 35.18 & 11.49 & 267,000 \\
\hline 1921 & 189 & 204 & 393 & 1,440 & 5,330 & 27.30 & 7.38 & 184,000 \\
\hline 1922 & 189 & 388 & 577 & 1,900 & 6,530 & 30.37 & 8.84 & 213,000 \\
\hline 1923 & 239 & 416 & 655 & 2,190 & 7,650 & 29.90 & 8.56 & 200,000 \\
\hline 1924 & 242 & 413 & 656 & 1,990 & 7,450 & 32.95 & 8.80 & 205,000 \\
\hline 1925 & 241 & 409 & 650 & 2,070 & 7,650 & 31.41 & 8.50 & 206,000 \\
\hline 1926 & 235 & 397 & 632 & 1,940 & 7,890 & 32.59 & 8.01 & 184,000 \\
\hline 1927 & 277 & 348 & 625 & 1,850 & 7,900 & 33.80 & 7.91 & 172,000 \\
\hline 1928 & 280 & 338 & 617 & 1,800 & 8,020 & 34.30 & 7.70 & 177,000 \\
\hline 1929 & 293 & 396 & 688 & 1,890 & 8,120 & 36.42 & 8.48 & 162,000 \\
\hline 1930 & 293 & 219 & 512 & 1,480 & 7,740 & 34.62 & 6.62 & 119,000 \\
\hline 1931 & 225 & 119 & 344 & 929 & 6,080 & 37.00 & 5.65 & 99,800 \\
\hline 1932 & 209 & 52 & 261 & 712 & 5,130 & 36.71 & 5.09 & 107,000 \\
\hline 1933 & 217 & 83 & 300 & 725 & 5,340 & 41.39 & 5.62 & 142,000 \\
\hline 1934 & 230 & 125 & 355 & 1,030 & 5,990 & 34.43 & 5.92 & 188,000 \\
\hline 1935 & 319 & 290 & 609 & 1,520 & 6,890 & 40.03 & 8.83 & 244,000 \\
\hline 1936 & 452 & 361 & 813 & 1,920 & 7,920 & 42.34 & 10.26 & 170,000 \\
\hline 1937 & 609 & 367 & 977 & 2,240 & 8,640 & 43.60 & 11.30 & 164,000 \\
\hline 1938 & 591 & 199 & 790 & 1,960 & 8,320 & 40.30 & 9.49 & 160,000 \\
\hline 1939 & 536 & 283 & 818 & 2,040 & 8,300 & 40.11 & 9.86 & 147,000 \\
\hline 1940 & 546 & 384 & 930 & 2,230 & 8,570 & 41.72 & 10.86 & 131,000 \\
\hline 1941 & 519 & 385 & 904 & 2,090 & 8,140 & 43.25 & 11.10 & 125,000 \\
\hline 1942 & 456 & 348 & 803 & 1,680 & 7,780 & 47.83 & 10.33 & 122,000 \\
\hline
\end{tabular}




\begin{tabular}{|c|c|c|c|c|c|c|c|c|}
\hline 1943 & 364 & 263 & 627 & 1,290 & 6,380 & 48.59 & 9.82 & 136,000 \\
\hline 1944 & 309 & 221 & 530 & 1,070 & 5,740 & 49.49 & 9.23 & 134,000 \\
\hline 1945 & 253 & 185 & 438 & 903 & 5,040 & 48.51 & 8.69 & 151,000 \\
\hline 1946 & 202 & 102 & 304 & 713 & 3,970 & 42.59 & 7.65 & 215,000 \\
\hline 1947 & 322 & 197 & 519 & 1,110 & 5,220 & 46.72 & 9.93 & 169,000 \\
\hline 1948 & 356 & 216 & 572 & 1,180 & 5,440 & 48.45 & 10.51 & 161,000 \\
\hline 1949 & 313 & 197 & 509 & 1,080 & 5,570 & 47.16 & 9.14 & 158,000 \\
\hline 1950 & 501 & 205 & 706 & 1,320 & 6,320 & 53.46 & 11.16 & 161,000 \\
\hline 1951 & 459 & 199 & 658 & 1,240 & 6,210 & 53.04 & 10.59 & 180,000 \\
\hline 1952 & 464 & 191 & 655 & 1,230 & 6,700 & 53.26 & 9.78 & 168,000 \\
\hline 1953 & 455 & 208 & 663 & 1,170 & 6,900 & 56.70 & 9.61 & 167,000 \\
\hline 1954 & 494 & 161 & 655 & 1,150 & 6,670 & 56.92 & 9.81 & 166,000 \\
\hline 1955 & 430 & 189 & 619 & 1,160 & 7,000 & 53.39 & 8.85 & 174,000 \\
\hline 1956 & 419 & 230 & 649 & 1,200 & 7,020 & 54.06 & 9.24 & 176,000 \\
\hline 1957 & 469 & 173 & 642 & 1,190 & 7,190 & 53.91 & 8.92 & 170,000 \\
\hline 1958 & 496 & 113 & 609 & 1,060 & 7,430 & 57.46 & 8.20 & 161,000 \\
\hline 1959 & 517 & 106 & 624 & 970 & 6,910 & 64.31 & 9.03 & 164,000 \\
\hline 1960 & 424 & 112 & 537 & 957 & 7,320 & 56.08 & 7.33 & 161,000 \\
\hline 1961 & 547 & 109 & 655 & 1,080 & 7,370 & 60.67 & 8.89 & 161,000 \\
\hline 1962 & 553 & 142 & 695 & 1,140 & 7,650 & 60.93 & 9.08 & 189,000 \\
\hline 1963 & 520 & 132 & 652 & 1,100 & 7,780 & 59.25 & 8.38 & 219,000 \\
\hline 1964 & 513 & 165 & 677 & 1,130 & 7,730 & 59.93 & 8.76 & 218,000 \\
\hline 1965 & 574 & 162 & 736 & 1,240 & 8,010 & 59.36 & 9.19 & 214,000 \\
\hline 1966 & 615 & 165 & 781 & 1,360 & 8,300 & 57.40 & 9.40 & 208,000 \\
\hline 1967 & 530 & 64 & 594 & 1,010 & 8,030 & 58.82 & 7.40 & 243,000 \\
\hline 1968 & 496 & 66 & 563 & 1,020 & 8,560 & 55.17 & 6.57 & 322,000 \\
\hline 1969 & 589 & 107 & 695 & 1,300 & 9,200 & 53.50 & 7.56 & 255,900 \\
\hline 1970 & 595 & 134 & 728 & 1,400 & 9,360 & 52.03 & 7.78 & 238,900 \\
\hline 1971 & 595 & 85 & 681 & 1,290 & 9,170 & 52.77 & 7.42 & 200,600 \\
\hline 1972 & 443 & 103 & 547 & 1,160 & 9,380 & 47.13 & 5.83 & 210,500 \\
\hline 1973 & 424 & 135 & 559 & 1,170 & 9,700 & 47.77 & 5.76 & 301,900 \\
\hline 1974 & 387 & 109 & 496 & 1,050 & 9,260 & 47.24 & 5.36 & 500,600 \\
\hline 1975 & 431 & 81 & 513 & 1,090 & 9,430 & 47.04 & 5.44 & 430,500 \\
\hline 1976 & 360 & 102 & 462 & 1,070 & 9,840 & 43.14 & 4.69 & 400,500 \\
\hline 1977 & 476 & 105 & 580 & 1,190 & 10,300 & 48.77 & 5.63 & 399,600 \\
\hline 1978 & 572 & 91 & 662 & 1,230 & 10,700 & 53.85 & 6.19 & 433,800 \\
\hline 1979 & 533 & 103 & 636 & 1,180 & 10,800 & 53.89 & 5.89 & 800,900 \\
\hline 1980 & 426 & 63 & 489 & 1,010 & 10,700 & 48.41 & 4.57 & $1,312,000$ \\
\hline 1981 & 515 & 93 & 608 & 1,270 & 11,200 & 47.84 & 5.43 & 606,200 \\
\hline 1982 & 461 & 192 & 653 & 1,250 & 11,500 & 52.25 & 5.68 & 431,700 \\
\hline 1983 & 550 & 178 & 728 & 1,350 & 12,100 & 53.89 & 6.01 & 601,900 \\
\hline 1984 & 587 & 176 & 763 & 1,390 & 13,100 & 54.87 & 5.82 & 410,800 \\
\hline 1985 & 586 & 125 & 710 & 1,230 & 13,100 & 57.75 & 5.42 & 299,200 \\
\hline 1986 & 349 & 148 & 497 & 1,070 & 13,000 & 46.45 & 3.82 & 261,500 \\
\hline 1987 & $\mathrm{~W}$ & 185 & 184 & 1,240 & 14,000 & 14.84 & 1.31 & 323,300 \\
\hline 1988 & 340 & 192 & 532 & 1,660 & 15,500 & 32.05 & 3.43 & 289,300 \\
\hline 1989 & 439 & 194 & 633 & 2,010 & 16,400 & 31.49 & 3.86 & 232,500 \\
\hline 1990 & 442 & 220 & 662 & 2,120 & 16,600 & 31.23 & 3.99 & 193,300 \\
\hline
\end{tabular}




\begin{tabular}{rrrrrrrrr}
1991 & 337 & 222 & 559 & 1,860 & 15,600 & 30.05 & 3.58 & 155,400 \\
1992 & 254 & 197 & 451 & 1,800 & 14,900 & 25.06 & 3.03 & 147,200 \\
1993 & 190 & 127 & 317 & 1,640 & 14,100 & 19.33 & 2.25 & 156,000 \\
1994 & 162 & 71 & 233 & 1,490 & 14,000 & 15.64 & 1.66 & 187,000 \\
1995 & 182 & 76 & 258 & 1,560 & 14,800 & 16.54 & 1.74 & 177,100 \\
1996 & 229 & 94 & 323 & 1,570 & 14,900 & 20.57 & 2.17 & 173,400 \\
1997 & 341 & $\mathrm{~W}$ & 341 & 2,180 & 16,000 & 15.64 & 2.13 & 159,700 \\
1998 & 447 & $\mathrm{~W}$ & 447 & 2,060 & 16,400 & 21.70 & 2.73 & 164,000 \\
1999 & 416 & $\mathrm{~W}$ & 416 & n.d. & n.d. & n.d. & n.d. & n.d. \\
2000 & 416 & $\mathrm{~W}$ & 416 & n.d. & n.d. & n.d. & n.d. & n.d. \\
2001 & $\mathrm{~W}$ & $\mathrm{~W}$ & $\mathrm{~W}$ & n.d. & n.d. & n.d. & n.d. & n.d. \\
\hline
\end{tabular}




\section{Talc}

Montana talc mining began in 1941 and became one of the nation's leading talc-producing states; however, much of the production data are proprietary and have been withheld from publication. Montana produced between 200,000 and 450,000 metric tons of talc per year in the years for which data are available (fig. 15A). U.S. production increased significantly from their previously stagnant levels once production of Montana talc began in the early 1940's. Prior to Montana's contribution to the talc industry, national production rarely topped 200,000 metric tons; however by 1979 , U.S. talc production rose to $1,300,000$ metric tons per year (fig. 15B). The significance of Montana's talc industry was highlighted in the late-1980s and early-1990s when the states production contributed as much as 40 percent to the nation's total (fig. 15C), and nearly 5 percent to the world's total (fig. 15D). No talc production is reported from Montana after 1993. There is no record of talc production from Idaho.
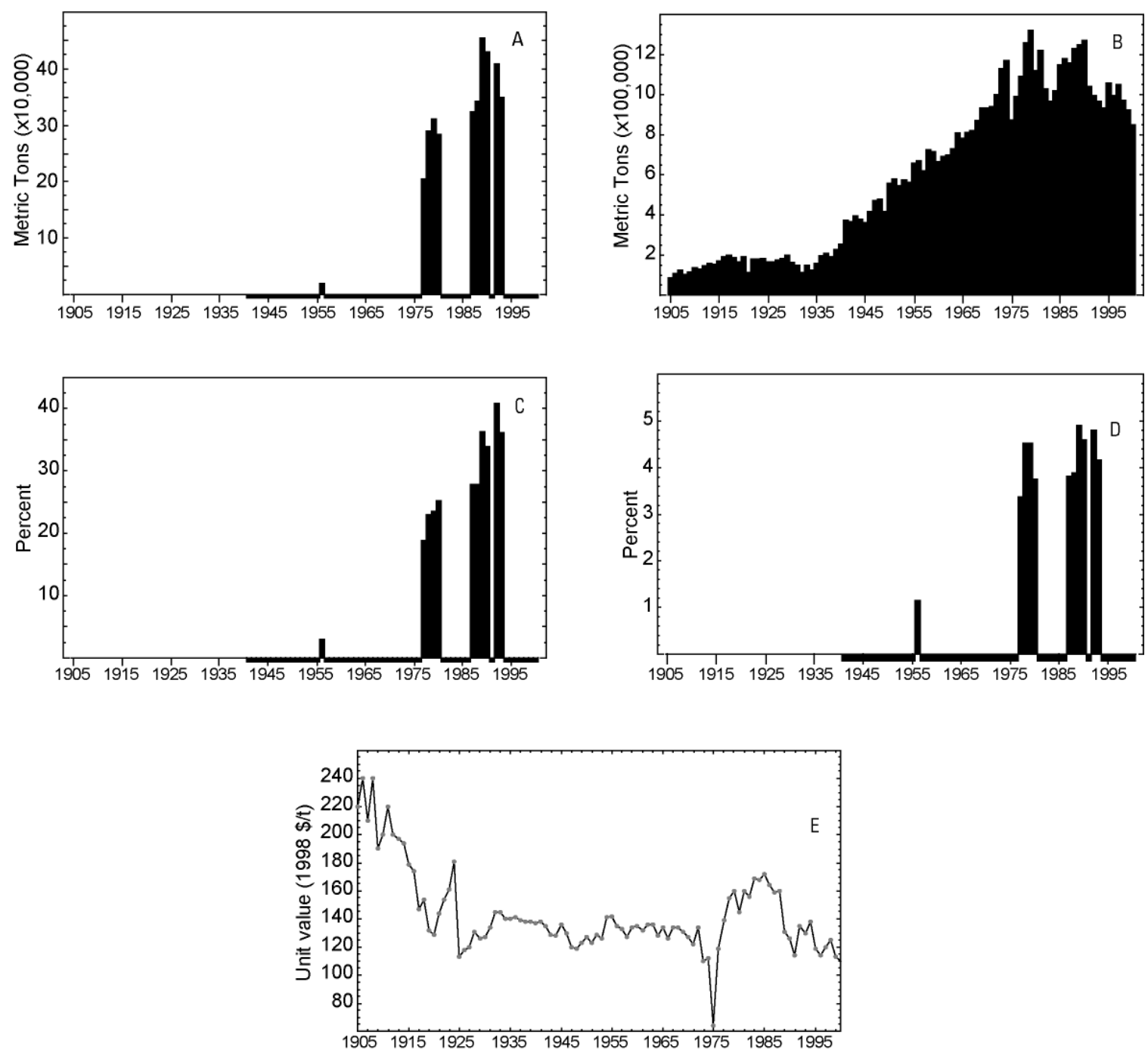

Figure 15. Talc production information from 1905-2001. A, annual production from Montana. B, annual U.S. domestic production of talc. E, the proportion of Montana talc production to U.S. production. F, the proportion of Montana talc production to world production. $\mathrm{G}$, the historical value of talc in constant 1998 dollars. Bars extending below the $\mathrm{x}$-axis represent data withheld from publication. 
Table 34. Talc production from Montana, the U.S., and the world, 1905-2000

[The table also summarizes the proportion of Montana production relative to the US and the world as well as the price for talc in constant 1998 dollars. National talc production figures for the years 1905-1920 represent talc and soapstone produced and sold in the U.S., but also include pyrophyllite from 1921-1940. National figures from 1941-1971 include talc, soapstone, and pyrophyllite produced from domestic mines, though after 1971 soapstone is not included. Data for state, US, and world production are given in metric tons; proportions of US and world production by Montana are given in percent; talc value is in US dollars. W, withheld; leaders ( $\_$) , no production]

\begin{tabular}{|c|c|c|c|c|c|c|}
\hline \multirow[b]{2}{*}{ Year } & \multicolumn{3}{|c|}{ Production } & \multicolumn{2}{|c|}{$\begin{array}{l}\text { Proportion of Montana } \\
\text { production to: }\end{array}$} & \multirow{2}{*}{$\begin{array}{r}\text { Talc value } \\
(1998 \$ / t\end{array}$} \\
\hline & Montana & US & World & US & World & \\
\hline 1905 & - - & 87,700 & 124,000 & _- & - - & 220 \\
\hline 1906 & -- & 109,000 & 151,000 & -- & -- & 240 \\
\hline 1907 & -- & 127,000 & 191,000 & -- & -- & 210 \\
\hline 1908 & -- & 106,000 & 160,000 & -- & -- & 240 \\
\hline 1909 & -- & 118,000 & 178,000 & -- & -- & 190 \\
\hline 1910 & -- & 137,000 & 202,000 & -- & -- & 200 \\
\hline 1911 & -- & 130,000 & 208,000 & -- & -- & 220 \\
\hline 1912 & -- & 144,000 & 171,000 & -- & -- & 200 \\
\hline 1913 & -- & 160,000 & 279,000 & -- & -- & 197 \\
\hline 1914 & -- & 156,000 & 213,000 & -- & -- & 194 \\
\hline 1915 & -- & 170,000 & 224,000 & -- & -- & 179 \\
\hline 1916 & -- & 193,000 & 257,000 & -- & -- & 174 \\
\hline 1917 & -- & 199,000 & 266,000 & -- & -- & 147 \\
\hline 1918 & -- & 189,000 & 252,000 & -- & -- & 154 \\
\hline 1919 & -- & 168,000 & 255,000 & -- & -- & 132 \\
\hline 1920 & -- & 191,000 & 322,000 & -- & -- & 129 \\
\hline 1921 & -- & 111,000 & 207,000 & -- & -- & 144 \\
\hline 1922 & -- & 180,000 & 353,000 & -- & -- & 154 \\
\hline 1923 & -- & 178,000 & 336,000 & -- & -- & 161 \\
\hline 1924 & -- & 185,000 & 375,000 & -- & -- & 181 \\
\hline 1925 & -- & 165,000 & 398,000 & -- & -- & 113 \\
\hline 1926 & -- & 165,000 & 344,000 & -- & -- & 118 \\
\hline 1927 & -- & 174,000 & 431,000 & -- & -- & 120 \\
\hline 1928 & -- & 184,000 & 389,000 & -- & -- & 131 \\
\hline 1929 & -- & 199,000 & 421,000 & -- & -- & 126 \\
\hline 1930 & -- & 163,000 & 364,000 & -- & -- & 127 \\
\hline 1931 & -- & 149,000 & 384,000 & -- & -- & 134 \\
\hline 1932 & -- & 112,000 & 331,000 & -- & -- & 145 \\
\hline 1933 & -- & 151,000 & 430,000 & -- & -- & 145 \\
\hline 1934 & -- & 126,000 & 399,000 & -- & -- & 140 \\
\hline 1935 & -- & 157,000 & 424,000 & -- & -- & 140 \\
\hline 1936 & -- & 196,000 & 472,000 & -- & -- & 141 \\
\hline 1937 & -- & 209,000 & 515,000 & -- & -- & 139 \\
\hline 1938 & -- & 193,000 & 420,000 & -- & -- & 138 \\
\hline 1939 & -- & 230,000 & 488,000 & -- & -- & 138 \\
\hline 1940 & -- & 255,000 & 664,000 & -- & -- & 137 \\
\hline
\end{tabular}




\begin{tabular}{|c|c|c|c|c|c|c|}
\hline 1941 & W & 376,000 & 840,000 & W & W & 138 \\
\hline 1942 & W & 366,000 & $1,170,000$ & W & W & 135 \\
\hline 1943 & W & 396,000 & $1,120,000$ & $\mathrm{~W}$ & $\mathrm{~W}$ & 129 \\
\hline 1944 & W & 379,000 & $1,010,000$ & $\mathrm{~W}$ & W & 128 \\
\hline 1945 & W & 364,000 & 840,000 & W & W & 136 \\
\hline 1946 & W & 418,000 & 950,000 & W & W & 130 \\
\hline 1947 & W & 469,000 & $1,060,000$ & $\mathrm{~W}$ & W & 120 \\
\hline 1948 & W & 479,000 & $1,300,000$ & W & W & 119 \\
\hline 1949 & W & 417,000 & $1,280,000$ & W & W & 123 \\
\hline 1950 & W & 559,000 & $1,430,000$ & W & W & 127 \\
\hline 1951 & W & 581,000 & $1,570,000$ & $\mathrm{~W}$ & $\mathrm{~W}$ & 123 \\
\hline 1952 & W & 545,000 & $1,410,000$ & W & W & 129 \\
\hline 1953 & W & 573,000 & $1,480,000$ & W & W & 126 \\
\hline 1954 & W & 562,000 & $1,470,000$ & W & W & 141 \\
\hline 1955 & W & 659,000 & $1,620,000$ & W & W & 142 \\
\hline 1956 & 20,137 & 670,000 & $1,750,000$ & 3.01 & 1.15 & 135 \\
\hline 1957 & W & 621,000 & $2,010,000$ & W & W & 133 \\
\hline 1958 & W & 724,000 & $1,910,000$ & W & W & 127 \\
\hline 1959 & W & 718,000 & $2,350,000$ & $\mathrm{~W}$ & W & 134 \\
\hline 1960 & W & 666,000 & $2,520,000$ & W & W & 135 \\
\hline 1961 & W & 691,000 & $2,710,000$ & W & W & 132 \\
\hline 1962 & W & 700,000 & $2,670,000$ & W & W & 136 \\
\hline 1963 & W & 729,000 & $2,990,000$ & W & W & 136 \\
\hline 1964 & W & 807,000 & $3,520,000$ & W & W & 128 \\
\hline 1965 & W & 783,000 & $3,570,000$ & W & W & 134 \\
\hline 1966 & W & 812,000 & $3,710,000$ & W & $\mathrm{W}$ & 126 \\
\hline 1967 & W & 819,000 & $3,960,000$ & W & W & 134 \\
\hline 1968 & W & 869,000 & $4,350,000$ & W & W & 134 \\
\hline 1969 & W & 933,000 & $4,680,000$ & W & W & 131 \\
\hline 1970 & W & 933,000 & $4,820,000$ & W & W & 127 \\
\hline 1971 & W & 941,000 & $4,740,000$ & W & W & 122 \\
\hline 1972 & W & $1,000,000$ & $4,830,000$ & W & W & 134 \\
\hline 1973 & W & $1,130,000$ & $5,400,000$ & W & W & 110 \\
\hline 1974 & W & $1,170,000$ & $5,810,000$ & W & W & 112 \\
\hline 1975 & W & 875,000 & $4,900,000$ & W & W & 64.0 \\
\hline 1976 & W & 991,000 & $5,270,000$ & W & W & 119 \\
\hline 1977 & 205,027 & $1,090,000$ & $6,090,000$ & 18.81 & 3.37 & 139 \\
\hline 1978 & 289,397 & $1,260,000$ & $6,400,000$ & 22.97 & 4.52 & 155 \\
\hline 1979 & 311,170 & $1,320,000$ & $6,870,000$ & 23.57 & 4.53 & 160 \\
\hline 1980 & 283,046 & $1,120,000$ & $7,540,000$ & 25.27 & 3.75 & 145 \\
\hline 1981 & W & $1,220,000$ & $7,270,000$ & W & W & 160 \\
\hline 1982 & W & $1,030,000$ & $7,060,000$ & W & W & 156 \\
\hline 1983 & W & 967,000 & $7,060,000$ & W & W & 169 \\
\hline 1984 & W & $1,020,000$ & $7,570,000$ & W & W & 168 \\
\hline 1985 & W & $1,150,000$ & $7,830,000$ & W & W & 172 \\
\hline 1986 & W & $1,180,000$ & $7,760,000$ & W & W & 164 \\
\hline 1987 & 323,173 & $1,160,000$ & $8,470,000$ & 27.86 & 3.82 & 159 \\
\hline 1988 & 342,730 & $1,230,000$ & $8,810,000$ & 27.86 & 3.89 & 160 \\
\hline
\end{tabular}




\begin{tabular}{rrrrrrr}
1989 & 453,978 & $1,250,000$ & $9,240,000$ & 36.32 & 4.91 & 131 \\
1990 & 430,125 & $1,270,000$ & $9,370,000$ & 33.87 & 4.59 & 126 \\
1991 & $\mathrm{~W}$ & $1,040,000$ & $9,060,000$ & $\mathrm{~W}$ & $\mathrm{~W}$ & 114 \\
1992 & 407,657 & 997,000 & $8,500,000$ & 40.89 & 4.80 & 135 \\
1993 & 349,559 & 968,000 & $8,420,000$ & 36.11 & 4.15 & 130 \\
1994 & $\mathrm{~W}$ & 935,000 & $8,260,000$ & $\mathrm{~W}$ & $\mathrm{~W}$ & 138 \\
1995 & $\mathrm{~W}$ & $1,060,000$ & $8,490,000$ & $\mathrm{~W}$ & $\mathrm{~W}$ & 119 \\
1996 & $\mathrm{~W}$ & 994,000 & $9,880,000$ & $\mathrm{~W}$ & $\mathrm{~W}$ & 114 \\
1997 & $\mathrm{~W}$ & $1,050,000$ & $11,100,000$ & $\mathrm{~W}$ & $\mathrm{~W}$ & 120 \\
1998 & $\mathrm{~W}$ & 971,000 & $10,100,000$ & $\mathrm{~W}$ & $\mathrm{~W}$ & 125 \\
1999 & $\mathrm{~W}$ & 925,000 & $10,000,000$ & $\mathrm{~W}$ & $\mathrm{~W}$ & 113 \\
2000 & $\mathrm{~W}$ & 851,000 & $9,640,000$ & $\mathrm{~W}$ & $\mathrm{~W}$ & 110 \\
\hline
\end{tabular}




\section{Tungsten}

Idaho tungsten production began in 1911; however, significant production was limited to four years from 1942 through 1945 (fig. 84). Over 4,200 metric tons were produced in 1943 alone; however, this was the only period of abundant tungsten mining in Idaho. A second, though much smaller, period of production during the early- to mid-1950's coincided with a U.S. government strategic materials stockpile program (Kramer, undated). Significant production in Montana was limited to the period between 1954 and 1957; however, the maximum quantity produced in Montana amounted to only one-fourth that of Idaho's most prolific years (fig. 85). Together, Idaho and Montana contributed over 1,600 metric tons of tungsten per year in the mid-1950's (fig.86). Production from both Idaho and Montana decreased when the U.S. government terminated the strategic materials program (Shedd, 1999). Tungsten mining in the region ended in 1983, though data are withheld for both states after 1972. No charts were produced for the proportion of regional tungsten mining production to U.S. or world tungsten production, due to the very small numbers involved.

Table 35. Selected events affecting U.S. tungsten production

\begin{tabular}{lll}
\hline Year & Event & Reference \\
\hline Early 1950's & $\begin{array}{l}\text { US Government begins program of stockpiling tungsten after Korean } \\
\text { conflict }\end{array}$ & Shedd, 1999 \\
1964 & $\begin{array}{l}\text { Increase in US production due to decreased imports from communist } \\
\text { countries }\end{array}$ & Shedd, 1999 \\
\hline
\end{tabular}
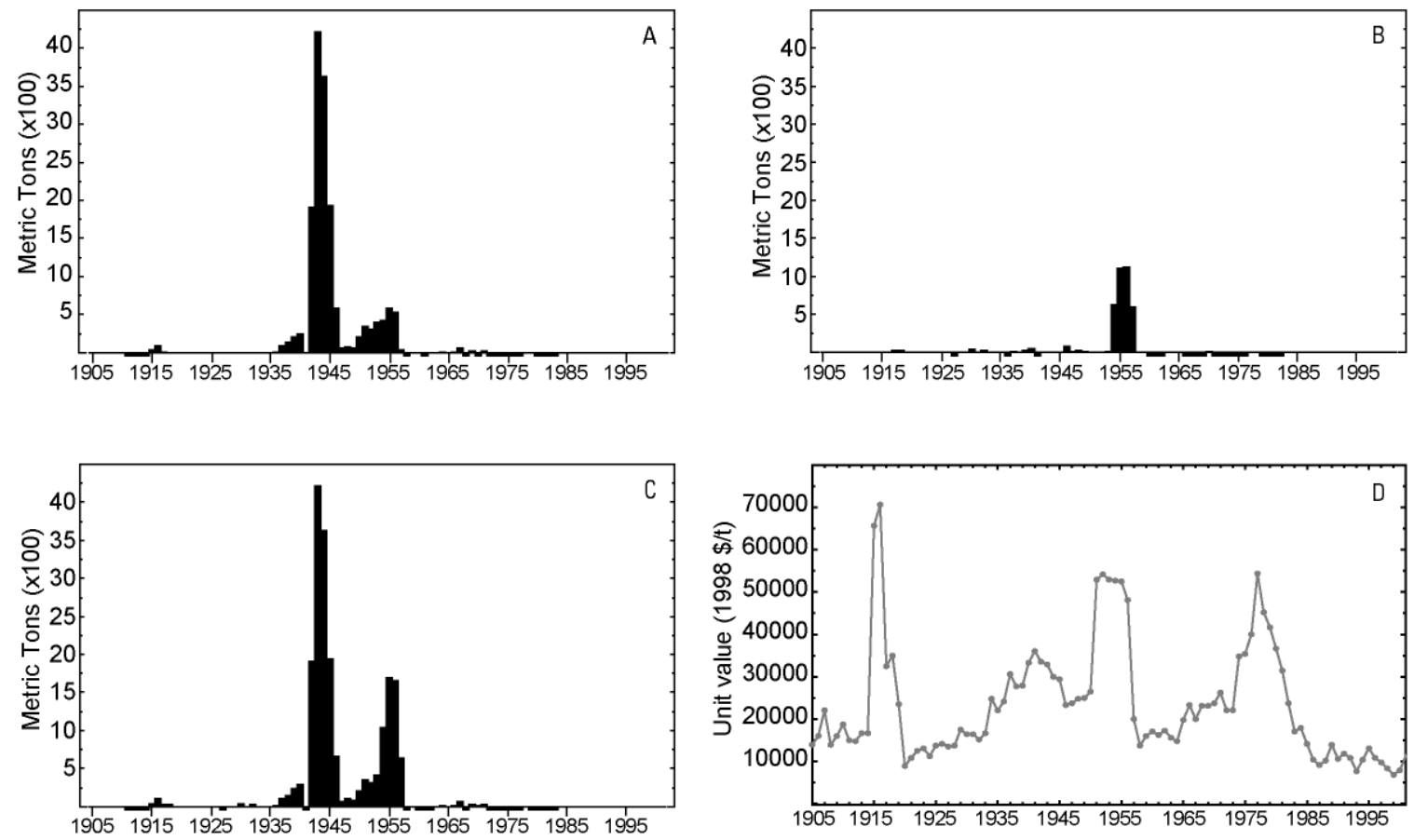

Figure 16. Tungsten production information from 1905-2001. A, annual production of tungsten concentrates from Idaho. $B$, annual production of tungsten concentrates from Montana. C, Idaho and Montana combined production. D, the historical value of tungsten in constant 1998 dollars. Bars extending below the $\mathrm{x}$-axis represent data withheld from publication.

Table 36. Tungsten (60-percent concentrate) production from Idaho and Montana, the U.S. and the world, 1905-2001

[The table also summarizes the proportion of Idaho and Montana production relative to the US and the world as well as the price for 
tungsten in constant 1998 dollars. The regional production figures represent tungsten concentrate containing 60-percent tungsten trioxide. Primary U.S. production of tungsten concentrates include domestically mined, imported, or stockpiled ore. Data for state, US, and world production are given in metric tons; proportions of US and world production by Idaho and Montana are given in percent; tungsten value is in US dollars. W, withheld; leaders ( _ _ ), no production]

\begin{tabular}{|c|c|c|c|c|c|c|c|c|}
\hline \multirow[b]{2}{*}{ Year } & \multicolumn{5}{|c|}{ Production } & \multicolumn{2}{|c|}{$\begin{array}{l}\text { Proportion of Idaho } \\
\text { and Montana } \\
\text { production to: }\end{array}$} & \multirow[b]{2}{*}{$\begin{array}{r}\text { Tungsten } \\
\text { value }(1998 \\
\$ / \mathbf{t}) \\
\end{array}$} \\
\hline & Idaho & Montana & $\begin{array}{r}\text { Idaho and } \\
\text { Montana } \\
\text { combined } \\
\end{array}$ & US & World & US & World & \\
\hline 1905 & -- & -- & -- & 347 & 1,700 & -- & -- & 14,000 \\
\hline 1906 & -- & -- & -- & 401 & 1,900 & -- & -- & 16,000 \\
\hline 1907 & -- & -- & -- & 708 & 2,600 & -- & -- & 22,000 \\
\hline 1908 & -- & -- & -- & 290 & 1,800 & -- & -- & 14,000 \\
\hline 1909 & -- & -- & -- & 699 & 2,500 & -- & -- & 16,000 \\
\hline 1910 & -- & -- & -- & 786 & 3,300 & -- & -- & 18,700 \\
\hline 1911 & $\mathrm{~W}$ & -- & W & 492 & 3,200 & W & W & 15,000 \\
\hline 1912 & W & -- & W & 574 & 4,200 & W & W & 14,800 \\
\hline 1913 & W & -- & W & 664 & 3,900 & W & W & 16,600 \\
\hline 1914 & W & -- & W & 427 & 3,500 & $\mathrm{~W}$ & W & 16,600 \\
\hline 1915 & 29.0 & -- & 29.0 & 1,010 & 5,200 & 0.03 & 0.01 & 65,700 \\
\hline 1916 & 91.6 & 0.9 & 92.5 & 2,560 & 10,000 & 0.04 & 0.01 & 70,600 \\
\hline 1917 & 1.8 & 14.5 & 16.3 & 2,650 & 12,300 & 0.01 & 0.00 & 32,600 \\
\hline 1918 & -- & 20.0 & 20.0 & 2,180 & 15,200 & 0.01 & 0.00 & 34,900 \\
\hline 1919 & -- & -- & -- & 141 & 7,000 & -- & -- & 23,600 \\
\hline 1920 & -- & -- & -- & 93.2 & 5,500 & -- & -- & 8,890 \\
\hline 1921 & -- & -- & -- & 0 & 2,300 & -- & -- & 10,900 \\
\hline 1922 & -- & -- & -- & 0 & 3,000 & -- & -- & 12,600 \\
\hline 1923 & -- & -- & -- & 104 & 3,300 & -- & -- & 13,200 \\
\hline 1924 & -- & -- & -- & 244 & 2,900 & -- & -- & 11,200 \\
\hline 1925 & -- & -- & -- & 514 & 4,900 & -- & -- & 13,700 \\
\hline 1926 & -- & -- & -- & 596 & 5,800 & -- & -- & 14,200 \\
\hline 1927 & -- & W & W & 503 & 4,400 & W & W & 13,500 \\
\hline 1928 & -- & -- & -- & 522 & 5,500 & -- & -- & 13,800 \\
\hline 1929 & -- & -- & -- & 358 & 7,500 & -- & -- & 17,400 \\
\hline 1930 & -- & 28.1 & 28.1 & 303 & 7,900 & 0.09 & 0.00 & 16,500 \\
\hline 1931 & -- & -- & -- & 606 & 6,400 & -- & -- & 16,400 \\
\hline 1932 & -- & 27.2 & 27.2 & 171 & 3,200 & 0.16 & 0.01 & 15,200 \\
\hline 1933 & -- & -- & -- & 386 & 5,900 & -- & -- & 16,700 \\
\hline 1934 & 0.9 & -- & 0.9 & 885 & 7,800 & 0.00 & 0.00 & 24,700 \\
\hline 1935 & -- & -- & -- & 1,030 & 10,700 & -- & -- & 22,100 \\
\hline 1936 & 10.0 & W & 10.0 & 1,130 & 11,800 & 0.01 & 0.00 & 24,200 \\
\hline 1937 & 89.8 & 12.7 & 102.5 & 1,510 & 18,500 & 0.07 & 0.01 & 30,700 \\
\hline 1938 & 139.7 & -- & 139.7 & 1,310 & 17,800 & 0.11 & 0.01 & 27,800 \\
\hline 1939 & 206.8 & 20.9 & 227.7 & 1,850 & 20,100 & 0.12 & 0.01 & 27,900 \\
\hline 1940 & 235.9 & 45.4 & 281.2 & 2,300 & 20,700 & 0.12 & 0.01 & 33,400 \\
\hline 1941 & -- & W & W & 2,830 & 23,900 & W & W & 36,100 \\
\hline 1942 & 1912.4 & -- & 1912.4 & 4,030 & 24,100 & 0.47 & 0.08 & 33,500 \\
\hline 1943 & 4216.7 & -- & 4216.7 & 5,160 & 28,600 & 0.82 & 0.15 & 32,900 \\
\hline 1944 & 3633.3 & -- & 3633.3 & 4,440 & 23,400 & 0.82 & 0.16 & 30,100 \\
\hline
\end{tabular}




\begin{tabular}{|c|c|c|c|c|c|c|c|c|}
\hline 1945 & 1932.3 & -- & 1932.3 & 2,390 & 10,900 & 0.81 & 0.18 & 29,300 \\
\hline 1946 & 581.5 & 76.2 & 657.7 & 2,240 & 9,040 & 0.29 & 0.07 & 23,400 \\
\hline 1947 & 55.3 & 3.6 & 59.0 & 1,340 & 13,700 & 0.04 & 0.00 & 23,800 \\
\hline 1948 & 78.0 & 25.4 & 103.4 & 1,740 & 17,800 & 0.06 & 0.01 & 24,700 \\
\hline 1949 & 59.9 & 8.2 & 68.0 & 1,190 & 15,800 & 0.06 & 0.00 & 25,100 \\
\hline 1950 & 201.4 & _- & 201.4 & 2,080 & 18,300 & 0.10 & 0.01 & 26,500 \\
\hline 1951 & 342.0 & 0.9 & 342.9 & 2,710 & 24,800 & 0.13 & 0.01 & 53,000 \\
\hline 1952 & 302.1 & -- & 302.1 & 3,290 & 32,700 & 0.09 & 0.01 & 54,100 \\
\hline 1953 & 400.1 & 12.7 & 412.8 & 4,140 & 34,400 & 0.10 & 0.01 & 53,000 \\
\hline 1954 & 427.3 & 615.1 & 1042.4 & 5,910 & 33,800 & 0.18 & 0.03 & 52,800 \\
\hline 1955 & 582.4 & 1098.6 & 1681.0 & 7,080 & 35,700 & 0.24 & 0.05 & 52,400 \\
\hline 1956 & 528.0 & 1115.9 & 1643.8 & 6,360 & 35,800 & 0.26 & 0.05 & 48,200 \\
\hline 1957 & 31.8 & 599.7 & 631.4 & 2,380 & 29,100 & 0.27 & 0.02 & 20,000 \\
\hline 1958 & W & -- & W & 1,640 & 24,200 & W & W & 13,800 \\
\hline 1959 & $\ldots$ & _- & _- & 1,580 & 26,400 & _- & _- & 16,000 \\
\hline 1960 & -_ & W & W & 3,160 & 31,200 & W & W & 17,100 \\
\hline 1961 & W & W & W & 3,560 & 33,000 & W & W & 16,200 \\
\hline 1962 & _- & W & W & 3,640 & 31,300 & W & W & 17,300 \\
\hline 1963 & _- & _- & _- & 2,440 & 27,100 & -- & -_ & 15,700 \\
\hline 1964 & 10.0 & _- & 10.0 & 3,990 & 28,100 & 0.00 & 0.00 & 14,800 \\
\hline 1965 & _- & $\ldots$ & _- & 3,430 & 27,000 & _- & - - & 19,700 \\
\hline 1966 & 1.8 & $\mathrm{~W}$ & 1.8 & 3,250 & 28,600 & 0.00 & 0.00 & 23,300 \\
\hline 1967 & 61.7 & W & 61.7 & 3,560 & 28,500 & 0.02 & 0.00 & 20,000 \\
\hline 1968 & W & W & W & 4,100 & 31,000 & W & W & 23,200 \\
\hline 1969 & 24.5 & W & 24.5 & 3,590 & 32,500 & 0.01 & 0.00 & 23,200 \\
\hline 1970 & W & 8.2 & 8.2 & 4,220 & 32,400 & 0.00 & 0.00 & 23,700 \\
\hline 1971 & 21.8 & W & 21.8 & 3,100 & 35,400 & 0.01 & 0.00 & 26,200 \\
\hline 1972 & W & W & W & 3,200 & 38,500 & W & W & 22,100 \\
\hline 1973 & W & W & W & 3,200 & 37,900 & W & W & 22,000 \\
\hline 1974 & W & W & W & 3,550 & 37,600 & W & W & 34,800 \\
\hline 1975 & W & W & W & 2,490 & 38,300 & W & W & 35,400 \\
\hline 1976 & W & W & W & 2,660 & 38,000 & W & W & 40,100 \\
\hline 1977 & W & -- & W & 2,730 & 41,100 & W & W & 54,300 \\
\hline 1978 & _- & -_ & -_ & 3,130 & 46,100 & -- & $\ldots$ & 45,300 \\
\hline 1979 & -_ & W & W & 3,010 & 48,600 & W & W & 41,600 \\
\hline 1980 & W & W & W & 2,740 & 52,000 & W & W & 36,600 \\
\hline 1981 & W & W & W & 3,550 & 50,300 & W & W & 31,500 \\
\hline 1982 & W & $\mathrm{W}$ & W & 1,580 & 47,000 & W & W & 23,700 \\
\hline 1983 & W & -- & W & 1,020 & 40,900 & W & W & 17,000 \\
\hline 1984 & -- & -- & -_ & 1,170 & 46,200 & -- & -- & 17,900 \\
\hline 1985 & -- & -_ & -- & 983 & 46,600 & -- & -- & 14,100 \\
\hline 1986 & -- & -- & -- & 817 & 43,500 & _- & -_ & 10,500 \\
\hline 1987 & -- & -- & -- & 34 & 42,500 & -- & -- & 9,110 \\
\hline 1988 & -- & -- & -- & -- & 50,900 & -- & -- & 10,300 \\
\hline 1989 & _- & _- & _- & _- & 51,000 & -_ & _- & 13,900 \\
\hline 1990 & -- & -- & -- & -- & 51,900 & -- & -- & 10,600 \\
\hline 1991 & -- & -- & -- & -- & 48,200 & -- & -- & 11,800 \\
\hline 1992 & - - & -_ & _- & _- & 42,900 & _- & $\ldots$ & 10,800 \\
\hline 1993 & -- & -- & -- & -- & 34,300 & -- & -- & 7,690 \\
\hline 1994 & -- & -- & _- & -_ & 34,000 & -- & -- & 10,400 \\
\hline
\end{tabular}




\begin{tabular}{rlllllllr}
1995 & -- & -- & -- & 0 & 38,500 & -- & -- & 13,200 \\
1996 & -- & -- & -- & 0 & 34,700 & -- & -- & 10,800 \\
1997 & -- & -- & -- & 0 & 33,200 & -- & -- & 9,810 \\
1998 & -- & -- & -- & 0 & 37,000 & -- & -- & 8,300 \\
1999 & -- & -- & -- & 0 & 37,700 & -- & -- & 6,920 \\
2000 & -- & -- & -- & 0 & 44,000 & -- & -- & 7,840 \\
2001 & -- & -- & -- & 0 & 45,300 & -- & -- & 11,500 \\
\hline
\end{tabular}




\section{Zinc}

The discovery and implementation of flotation as a more efficient method of recovering ore brought renewed interest in zinc mining to the region in the early 1900s. After two sharp declines in zinc production in 1921 and 1932 due to low prices, Idaho doubled its earlier output records and rose to around 80,000 metric tons of zinc per year (fig. 17A). Zinc production in Idaho decreased after 1950 and has been relatively insignificant since 1981. Montana also has shown abundant zinc resources, most notably in the Butte area where zinc has historically been the second most common commodity produced after copper (Miller, R.N., 1973). Montana's zinc industry peaked in 1916 when it produced 104,000 metric tons, while production rates in the years following have seen considerable fluctuations (fig. 17B). The highly variable production from the 1920 s through the 1960 s typically ranged from 10,000 to 80,000 metric tons; however, this declined to just dozens of tons after zinc mining ceased in Butte in 1966 (Miller, R.N., 1973). In 1987, Montana Tunnels mine began operations and the state has consistently produced about 20,000 metric tons per year. Together, Idaho and Montana show a varied production history, though the overall amount of refined zinc has stabilized in recent years at about 20,000 to 25,000 metric tons, down from the peak production in 1951 of 148,000 metric tons (fig. 17C). Large decreases in U.S. production, down from 900,000 to about 200,000 metric tons, show similar trends on a national scale to that of the region (fig. 17D). Idaho and Montana together have produced as much as 26 percent of the nation's zinc (fig. 17E) and 16 percent of the world's zinc (fig. 17F), though in recent years, their percentage has declined to 10 percent and less than 0.3 percent, respectively.

Table 37. Selected events affecting regional zinc production

\begin{tabular}{lll}
\hline Year & Event & Reference \\
\hline 1906 & Success mine begins recovery of zinc concentrate by gravity methods & this study \\
1910 & Morning mill begins experiments with flotation to recover zinc & this study \\
1917 & Bunker Hill Smelter begins operation & Chapman, 1994 \\
1917 & Great Falls electrolytic zinc plant opens & Miller, R.N., 1973 \\
1921 & Butte mines temporarily shut down because of low copper prices & Everett, 2002 \\
1928 & Sullivan Mining Company opens electrolytic zinc plant & this study \\
1930 & Severe curtailment of mining operations due to collapse of metals & this study \\
& prices & \\
1937 & Bunker Hill last mine to convert to all-through flotation & Art Bookstrom, 2004 \\
& & written commun. \\
1945 & Production decreases due to wartime labor shortage & this study \\
1949 & Strike at Bunker Hill caused smelter shutdown for 15 days & Chapman, 1994 \\
1955 & Lucky Friday mine opened & this study \\
1959 & Strike results in the closure of Anselmo mine near Butte & Shovers and others, 1991 \\
1960 & 200 day-long strike at Bunker Hill & Chapman, 1994 \\
$1962-67$ & Block cave mining for low-grade ore in the Badger mine & Miller, R.N., 1973 \\
1966 & Butte zinc mining discontinued because of low price, high costs & Miller, R.N., 1973 \\
1981 & Bunker Hill mine, mill, and smelter closed & Chapman, 1994 \\
1987 & Montana Tunnels mine opens & this study \\
1997 & Hecla begins production from Gold Hunter via Lucky Friday mine & this study \\
\hline
\end{tabular}

Table 38. Selected events affecting U.S. zinc production

\begin{tabular}{lll}
\hline Year & Event & Reference \\
\hline 1906 & Froth flotation comes into widespread use & Kramer, undated \\
1960 & Production begins at Viburnum Trend in Missouri & Kramer, undated \\
$1971-73$ & Price controls keeps value down & Plachy, 1999 \\
1973 & Price controls removed, price of zinc increases dramatically & Plachy, 1999 \\
1982 & Introduction of zinc penny & Plachy, 1999 \\
\hline
\end{tabular}



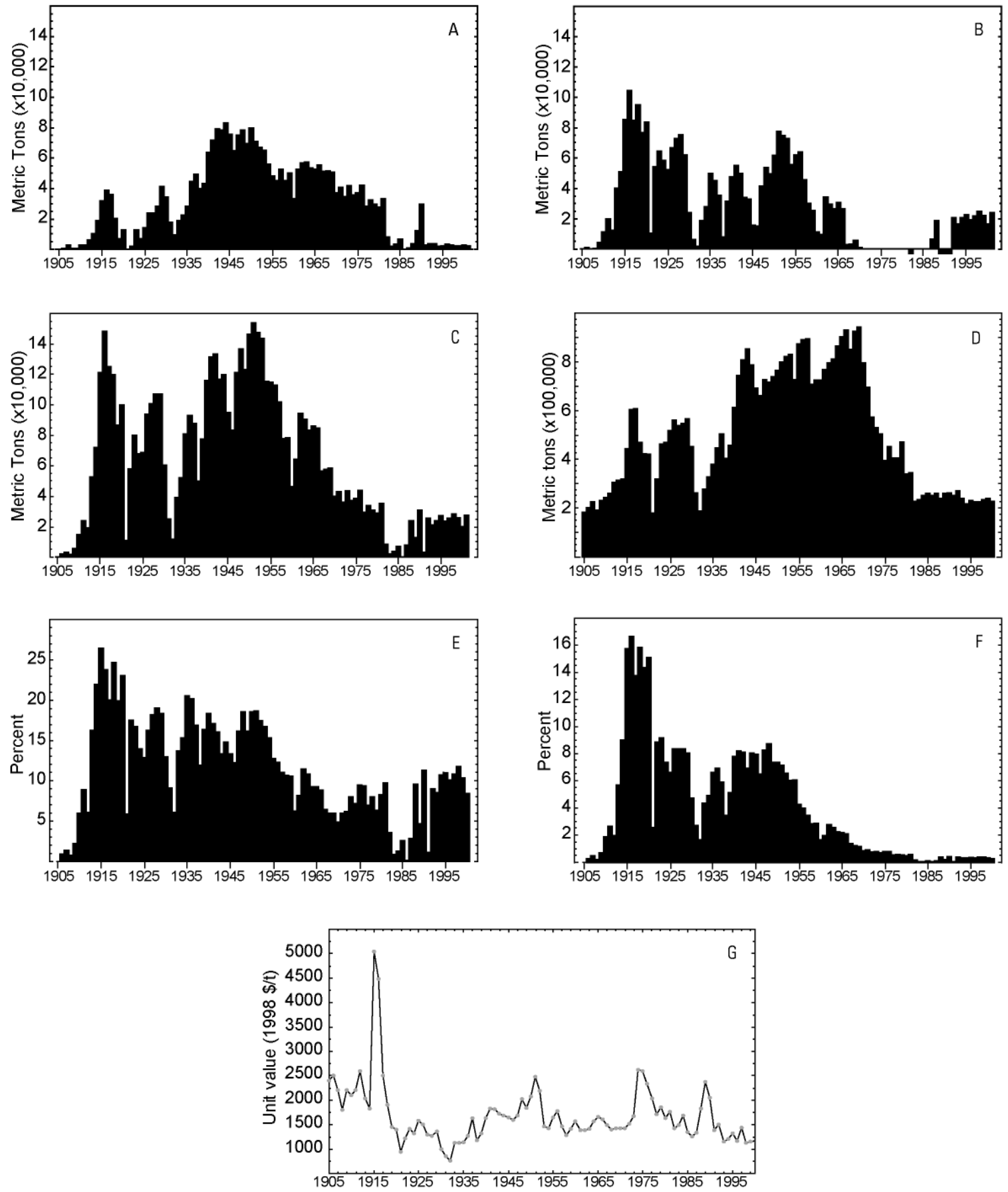

Figure 17. Zinc production information from 1905-2001. A, annual production from Idaho. B, annual production from Montana. C, Idaho and Montana combined production. D, annual U.S. primary production of zinc. E, the proportion of regional zinc production to U.S. production. $F$, the proportion of regional zinc production to world production. $G$, the historical value of zinc in constant 1998 dollars. Bars extending below the $\mathrm{x}$-axis represent data withheld from publication. 
Table 39. Zinc production from Idaho and Montana, the U.S., and the world, 1905-2001

[The table also summarizes the proportions of Idaho and Montana production relative to the US and the world as well as the price for zinc in constant 1998 dollars. Data for state, US, and world production are given in metric tons; proportions of US and world production by Idaho and Montana are given in percent; zinc value is in US dollars. W, withheld; n.d., no data; leaders ( _ - ), no production]

\begin{tabular}{|c|c|c|c|c|c|c|c|c|}
\hline \multirow[b]{2}{*}{ Year } & \multicolumn{5}{|c|}{ Production } & \multicolumn{2}{|c|}{$\begin{array}{l}\text { Proportion of Idaho } \\
\text { and Montana } \\
\text { production to: }\end{array}$} & \multirow[b]{2}{*}{$\begin{array}{r}\text { Zinc value } \\
(1998 \$ / t) \\
\end{array}$} \\
\hline & Idaho & Montana & $\begin{array}{r}\text { Montana } \\
\text { combined }\end{array}$ & US & World & US & World & \\
\hline 1905 & -- & -- & -- & 185,000 & 660,000 & n.d. & n.d. & 2,400 \\
\hline 1906 & 520 & 1,284 & 1,804 & 204,000 & 704,000 & 0.88 & 0.26 & 2,500 \\
\hline 1907 & 3,182 & -- & 3,182 & 227,000 & 738,000 & 1.40 & 0.43 & 2,200 \\
\hline 1908 & 527 & 816 & 1,344 & 191,000 & 723,000 & 0.70 & 0.19 & 1,800 \\
\hline 1909 & 817 & 4,287 & 5,104 & 232,000 & 775,000 & 2.20 & 0.66 & 2,200 \\
\hline 1910 & 3,133 & 11,257 & 14,390 & 244,000 & 810,000 & 5.90 & 1.78 & 2,100 \\
\hline 1911 & 2,885 & 20,063 & 22,948 & 260,000 & 895,000 & 8.83 & 2.56 & 2,200 \\
\hline 1912 & 6,308 & 12,210 & 18,518 & 307,000 & 971,000 & 6.03 & 1.91 & 2,600 \\
\hline 1913 & 10,512 & 40,223 & 50,734 & 314,000 & 915,000 & 16.16 & 5.54 & 2,030 \\
\hline 1914 & 19,057 & 50,613 & 69,669 & 320,000 & 795,000 & 21.77 & 8.76 & 1,830 \\
\hline 1915 & 31,822 & 84,889 & 116,711 & 444,000 & 760,000 & 26.29 & 15.36 & 5,050 \\
\hline 1916 & 39,239 & 103,992 & 143,231 & 606,000 & 882,000 & 23.64 & 16.24 & 4,480 \\
\hline 1917 & 36,222 & 84,488 & 120,709 & 607,000 & 901,000 & 19.89 & 13.40 & 2,500 \\
\hline 1918 & 20,485 & 94,919 & 115,405 & 470,000 & 747,000 & 24.55 & 15.45 & 1,900 \\
\hline 1919 & 7,255 & 76,551 & 83,806 & 423,000 & 598,000 & 19.81 & 14.01 & 1,450 \\
\hline 1920 & 12,670 & 83,616 & 96,286 & 420,000 & 655,000 & 22.93 & 14.70 & 1,400 \\
\hline 1921 & 15 & 10,558 & 10,573 & 182,000 & 428,000 & 5.81 & 2.47 & 945 \\
\hline 1922 & 1,864 & 54,010 & 55,874 & 321,000 & 647,000 & 17.41 & 8.64 & 1,220 \\
\hline 1923 & 12,679 & 64,166 & 76,845 & 463,000 & 858,000 & 16.60 & 8.96 & 1,410 \\
\hline 1924 & 6,958 & 58,277 & 65,235 & 469,000 & 910,000 & 13.91 & 7.17 & 1,320 \\
\hline 1925 & 14,170 & 52,307 & 66,477 & 520,000 & $1,030,000$ & 12.78 & 6.45 & 1,580 \\
\hline 1926 & 23,866 & 66,862 & 90,727 & 561,000 & $1,110,000$ & 16.17 & 8.17 & 1,500 \\
\hline 1927 & 24,293 & 72,786 & 97,079 & 538,000 & $1,190,000$ & 18.04 & 8.16 & 1,290 \\
\hline 1928 & 28,362 & 75,143 & 103,505 & 547,000 & $1,270,000$ & 18.92 & 8.15 & 1,270 \\
\hline 1929 & 41,436 & 61,849 & 103,286 & 567,000 & $1,320,000$ & 18.22 & 7.82 & 1,360 \\
\hline 1930 & 34,155 & 23,969 & 58,124 & 452,000 & $1,260,000$ & 12.86 & 4.61 & 990 \\
\hline 1931 & 17,753 & 6,121 & 23,874 & 265,000 & 904,000 & 9.01 & 2.64 & 857 \\
\hline 1932 & 9,301 & 1,993 & 11,294 & 188,000 & 709,000 & 6.01 & 1.59 & 762 \\
\hline 1933 & 19,022 & 18,801 & 37,823 & 279,000 & 892,000 & 13.56 & 4.24 & 1,120 \\
\hline 1934 & 22,498 & 27,870 & 50,368 & 330,000 & $1,060,000$ & 15.26 & 4.75 & 1,120 \\
\hline 1935 & 28,171 & 49,697 & 77,869 & 382,000 & $1,210,000$ & 20.38 & 6.44 & 1,140 \\
\hline 1936 & 44,544 & 45,103 & 89,647 & 446,000 & $1,330,000$ & 20.10 & 6.74 & 1,270 \\
\hline 1937 & 49,169 & 35,533 & 84,703 & 505,000 & $1,470,000$ & 16.77 & 5.76 & 1,630 \\
\hline 1938 & 39,944 & 8,023 & 47,967 & 405,000 & $1,420,000$ & 11.84 & 3.38 & 1,180 \\
\hline 1939 & 43,136 & 31,570 & 74,706 & 460,000 & $1,500,000$ & 16.24 & 4.98 & 1,320 \\
\hline 1940 & 64,049 & 47,707 & 111,756 & 613,000 & $1,470,000$ & 18.23 & 7.60 & 1,630 \\
\hline 1941 & 71,745 & 55,076 & 126,821 & 746,000 & $1,590,000$ & 17.00 & 7.98 & 1,830 \\
\hline 1942 & 79,159 & 49,637 & 128,796 & 809,000 & $1,630,000$ & 15.92 & 7.90 & 1,820 \\
\hline 1943 & 78,661 & 34,116 & 112,777 & 855,000 & $1,670,000$ & 13.19 & 6.75 & 1,720 \\
\hline
\end{tabular}




\begin{tabular}{|c|c|c|c|c|c|c|c|c|}
\hline 1944 & 82,893 & 32,774 & 115,667 & 789,000 & $1,470,000$ & 14.66 & 7.87 & 1,690 \\
\hline 1945 & 75,718 & 15,788 & 91,506 & 694,000 & $1,180,000$ & 13.19 & 7.75 & 1,650 \\
\hline 1946 & 64,871 & 15,214 & 80,085 & 661,000 & $1,260,000$ & 12.12 & 6.36 & 1,600 \\
\hline 1947 & 75,360 & 41,440 & 116,800 & 728,000 & $1,450,000$ & 16.04 & 8.06 & 1,690 \\
\hline 1948 & 78,261 & 53,611 & 131,872 & 715,000 & $1,550,000$ & 18.44 & 8.51 & 2,020 \\
\hline 1949 & 69,451 & 49,166 & 118,616 & 739,000 & $1,660,000$ & 16.05 & 7.15 & 1,840 \\
\hline 1950 & 79,734 & 61,397 & 141,131 & 765,000 & $1,970,000$ & 18.45 & 7.16 & 2,070 \\
\hline 1951 & 70,871 & 77,612 & 148,483 & 800,000 & $2,140,000$ & 18.56 & 6.94 & 2,480 \\
\hline 1952 & 67,420 & 74,558 & 141,979 & 821,000 & $2,230,000$ & 17.29 & 6.37 & 2,190 \\
\hline 1953 & 65,457 & 72,822 & 138,279 & 831,000 & $2,360,000$ & 16.64 & 5.86 & 1,460 \\
\hline 1954 & 55,818 & 55,296 & 111,114 & 728,000 & $1,880,000$ & 15.26 & 5.91 & 1,430 \\
\hline 1955 & 48,366 & 62,223 & 110,589 & 874,000 & $2,660,000$ & 12.65 & 4.16 & 1,650 \\
\hline 1956 & 44,962 & 63,976 & 108,937 & 892,000 & $2,810,000$ & 12.21 & 3.88 & 1,780 \\
\hline 1957 & 52,464 & 45,832 & 98,296 & 894,000 & $2,900,000$ & 11.00 & 3.39 & 1,460 \\
\hline 1958 & 45,111 & 30,154 & 75,264 & 709,000 & $2,730,000$ & 10.62 & 2.76 & 1,280 \\
\hline 1959 & 50,530 & 25,264 & 75,794 & 725,000 & $2,740,000$ & 10.45 & 2.77 & 1,410 \\
\hline 1960 & 33,386 & 11,386 & 44,772 & 725,000 & $2,790,000$ & 6.18 & 1.60 & 1,570 \\
\hline 1961 & 52,885 & 9,310 & 62,195 & 768,000 & $3,250,000$ & 8.10 & 1.91 & 1,390 \\
\hline 1962 & 57,031 & 34,181 & 91,213 & 798,000 & $3,410,000$ & 11.43 & 2.67 & 1,380 \\
\hline 1963 & 57,396 & 29,884 & 87,280 & 810,000 & $3,490,000$ & 10.78 & 2.50 & 1,410 \\
\hline 1964 & 53,795 & 26,362 & 80,157 & 866,000 & $3,690,000$ & 9.26 & 2.17 & 1,570 \\
\hline 1965 & 52,648 & 30,651 & 83,299 & 902,000 & $3,950,000$ & 9.23 & 2.11 & 1,660 \\
\hline 1966 & 55,336 & 26,418 & 81,754 & 930,000 & $4,080,000$ & 8.79 & 2.00 & 1,610 \\
\hline 1967 & 51,282 & 3,031 & 54,313 & 852,000 & $4,130,000$ & 6.37 & 1.32 & 1,490 \\
\hline 1968 & 51,935 & 3,427 & 55,363 & 926,000 & $4,630,000$ & 5.98 & 1.20 & 1,400 \\
\hline 1969 & 50,712 & 5,573 & 56,285 & 944,000 & $4,970,000$ & 5.96 & 1.13 & 1,430 \\
\hline 1970 & 37,242 & 1,322 & 38,564 & 796,000 & $4,830,000$ & 4.84 & 0.80 & 1,420 \\
\hline 1971 & 40,895 & 327 & 41,222 & 695,000 & $4,740,000$ & 5.93 & 0.87 & 1,430 \\
\hline 1972 & 35,061 & 11 & 35,071 & 574,000 & $5,130,000$ & 6.11 & 0.68 & 1,520 \\
\hline 1973 & 41,828 & 66 & 41,894 & 529,000 & $5,330,000$ & 7.92 & 0.79 & 1,670 \\
\hline 1974 & 35,806 & 123 & 35,930 & 504,000 & $5,610,000$ & 7.13 & 0.64 & 2,620 \\
\hline 1975 & 37,128 & 100 & 37,228 & 397,000 & $5,010,000$ & 9.38 & 0.74 & 2,600 \\
\hline 1976 & 42,263 & 58 & 42,321 & 453,000 & $5,610,000$ & 9.34 & 0.75 & 2,340 \\
\hline 1977 & 28,121 & 79 & 28,200 & 408,000 & $5,810,000$ & 6.91 & 0.49 & 2,040 \\
\hline 1978 & 32,353 & 79 & 32,432 & 407,000 & $5,880,000$ & 7.97 & 0.55 & 1,710 \\
\hline 1979 & 29,660 & 104 & 29,764 & 472,000 & $6,260,000$ & 6.31 & 0.48 & 1,850 \\
\hline 1980 & 27,722 & 71 & 27,793 & 340,000 & $6,050,000$ & 8.17 & 0.46 & 1,630 \\
\hline 1981 & 33,667 & 25 & 33,692 & 347,000 & $6,080,000$ & 9.71 & 0.55 & 1,760 \\
\hline 1982 & 8,108 & W & 8,108 & 228,000 & $5,890,000$ & 3.56 & 0.14 & 1,430 \\
\hline 1983 & 1,970 & -- & 1,970 & 236,000 & $6,250,000$ & 0.83 & 0.03 & 1,490 \\
\hline 1984 & 3,330 & -- & 3,330 & 253,000 & $6,530,000$ & 1.32 & 0.05 & 1,680 \\
\hline 1985 & 6,707 & -- & 6,707 & 261,000 & $6,790,000$ & 2.57 & 0.10 & 1,350 \\
\hline 1986 & 351 & -- & 351 & 253,000 & $6,690,000$ & 0.14 & 0.01 & 1,250 \\
\hline 1987 & 897 & 6,533 & 7,430 & 261,000 & $7,020,000$ & 2.85 & 0.11 & 1,330 \\
\hline 1988 & 3,972 & 18,935 & 22,907 & 241,000 & $7,160,000$ & 9.50 & 0.32 & 1,830 \\
\hline 1989 & 12,204 & W & 12,204 & 260,000 & $7,240,000$ & 4.69 & 0.17 & 2,380 \\
\hline 1990 & 29,518 & W & 29,518 & 263,000 & $7,180,000$ & 11.22 & 0.41 & 2,050 \\
\hline 1991 & 2,870 & $\mathrm{~W}$ & 2,870 & 253,000 & $7,310,000$ & 1.13 & 0.04 & 1,390 \\
\hline 1992 & 3,820 & 20,588 & 24,408 & 272,000 & $7,260,000$ & 8.97 & 0.34 & 1,500 \\
\hline 1993 & 3,978 & 16,371 & 20,349 & 240,000 & $7,360,000$ & 8.48 & 0.28 & 1,150 \\
\hline
\end{tabular}




\begin{tabular}{rrrrrrrrr}
1994 & 2,205 & 21,000 & 23,205 & 217,000 & $7,330,000$ & 10.69 & 0.32 & 1,200 \\
1995 & 2,721 & 22,700 & 25,421 & 232,000 & $7,280,000$ & 10.96 & 0.35 & 1,320 \\
1996 & 3,314 & 19,400 & 22,714 & 226,000 & $7,480,000$ & 10.05 & 0.30 & 1,170 \\
1997 & 2,874 & 21,500 & 24,374 & 226,000 & $7,530,000$ & 10.78 & 0.32 & 1,440 \\
1998 & 2,402 & 24,900 & 27,302 & 234,000 & $7,690,000$ & 11.67 & 0.36 & 1,130 \\
1999 & 2,654 & 22,200 & 24,854 & 241,000 & $8,040,000$ & 10.31 & 0.31 & 1,150 \\
2000 & 2,819 & 16,400 & 19,219 & 228,000 & $8,730,000$ & 8.43 & 0.22 & 1,160 \\
2001 & 2,538 & 24,000 & 26,538 & n.d. & n.d. & n.d. & n.d. & n.d. \\
\hline
\end{tabular}




\section{References}

ASARCO, 1998, ASARCO 1997 annual report. ONLINE. Available: URL: http://www.asarco.com/AnnualReport/ [Accessed July 30, 2003].

ASARCO, 2000, ASARCO 1999 annual report. ONLINE. Available: URL: http://www.asarco.com/MiningMetals/other/prodstat.html [Accessed July 30, 2003].

Carlin, J.F., Jr., 1999, Antimony, in Metal prices in the United States through 1998: U.S. Geological Survey, p.5-6, ONLINE. Available: URL: http://minerals.usgs.gov/minerals/pubs/metal prices/ [Accessed October 5, 2005].

Chapman, Ray, 1994, “Uncle Bunker”: Memories in Words and Pictures: Kellogg, Idaho, Chapman Publishing, 162 p.

Cox, D.P., Schmidt, R.G., Vine, J.D., Kirkemo, Harold, Tourtelot, E.B., and Fleischer, Michael, 1973, Copper, in Brobst, D.A., and Pratt, W.P., eds., United States Mineral Resources: U. S. Geological Survey Professional Paper 820, p. 163190.

Edelstein, Daniel, 1999, Copper, in Metal prices in the United States through 1998: U.S. Geological Survey, p.39-42, ONLINE. Available: URL: http://minerals.usgs.gov/minerals/pubs/metal_prices/ [Accessed October 5, 2005].

Everett, George, 2002, When toil meant trouble: Butte's labor heritage. ONLINE. Available: URL: http://www.butteamerica.com/labor.htm [Accessed July 13, 2004].

Goonan, Thomas, 2002, Policy - a factor shaping minerals supply and demand: U.S. Geological Survey Open File Report $02-418$, p. 37

Heyl, A.V., Hall, W.E., Weissenborn, A.E., Stager, H.K., Puffet W.P., and Reed, B.L., 1973, Silver, in Brobst, D.A., and Pratt, W.P., eds., United States Mineral Resources: U. S. Geological Survey Professional Paper 820, p. 581-603.

Hilliard, H.E., 1999a, Platinum-Group Metals, in Metal prices in the United States through 1998: U.S. Geological Survey, p.99-104, ONLINE. Available: URL: http://minerals.usgs.gov/minerals/pubs/metal prices/ [Accessed October 5, 2005].

Hilliard, H.E., 1999b, Silver, in Metal prices in the United States through 1998: U.S. Geological Survey, p.139-142, ONLINE. Available: URL: http://minerals.usgs.gov/minerals/pubs/metal prices/ [Accessed October 5, 2005].

Jasinsky, S.M, Lee, W.H., and Causey, J.D., 2004, The history of production of the Western Phosphate Field, Chap. 3 in Hein, J.R., ed., Life cycle of the Phosphoria Formation; from deposition to the post-mining environment: Elsevier, Handbook of Exploration and Environmental Geochemistry, Vol. 8, p. 45-71.

Kelly, Thomas; Buckingham, David, DiFrancesco, Carl, Porter, Kenneth, Goonan, Thomas, Szmopek, John, Berry, Cryus, and Crane, Melissa, 2001, Historical statistics for mineral commodities in the United States: U.S. Geological Survey Open File Report 01-006. ONLINE. Available: URL http://minerals.usgs.gov/minerals/pubs/of01006/index.html [Accessed June 27, 2003].

Kiilsgaard, T.H., 1964, Silver, lead, and zinc, in Mineral and water resources of Idaho: U.S. Geological Survey, Special Report no. 1, p.181-194.

Kramer, D.A., undated, Events affecting the U.S. nonfuel minerals industry, $1900-2000$. ONLINE. Available: URL http://minerals.usgs.gov/minerals/pubs/commodity/timeline [Accessed June 26, 2003]. 
Miller, M.H., 1973, Antimony, in Brobst, D.A., and Pratt, W.P., eds., United States Mineral Resources: U. S. Geological Survey Professional Paper 820, p. 45-50.

Miller, R.N., 1973, Production history of the Butte District and geological function, past and present, in, Miller, R.N. ed., Guidebook for the Butte field meeting of Society of Economic Geologists: Anaconda Co., Butte, Montana, Aug. 18-21. Section F.

Page, N.J, Clark, A.J., Desborough, G.A., and Parker, R.L., 1973, Platinum-group metals, in Brobst, D.A., and Pratt, W.P., eds., United States Mineral Resources: U. S. Geological Survey Professional Paper 820, p. 537-545.

Plachy, Jozef, 1999, Zinc, in Metal prices in the United States through 1998: U.S. Geological Survey, p.171-173, 0NLINE. Available: URL: http://minerals.usgs.gov/minerals/pubs/metal_prices/ [Accessed October 5, 2005].

Reese, R.G. Jr., 1999, Mercury, in Metal prices in the United States through 1998: U.S. Geological Survey, p, 87-88, ONLINE. Available: URL: http://minerals.usgs.gov/minerals/pubs/metal prices/ [Accessed October 5, 2005].

Shedd, K.B., 1999, Tungsten, in Metal prices in the United States through 1998: U.S. Geological Survey, p.171-173, ONLINE. Available: URL: http://minerals.usgs.gov/minerals/pubs/metal_prices/ [Accessed October 5, 2005].

Shovers, Brian, Fiege, Mark, Martin, Dale, and Quivik, Fred, 1991, Butte and Anaconda revisited, an overview of earlyday mining and smelting in Montana: Montana Bureau of Mines and Geology Special Publication 99, $64 \mathrm{p.}$

Smith, G.R., 1999, Lead, in Metal prices in the United States through 1998: U.S. Geological Survey, p.73-75, 0NLINE. Available: URL: http://minerals.usgs.gov/minerals/pubs/metal_prices/ [Accessed October 5, 2005]. 


\section{Appendix 1}

\section{Conversion Factors}

All units in production tables in this report are reported as metric tons. The original data from USBM and USGS were reported in a variety of units depending on the commodity and standards for the time period when production was reported. The conversion factors to standardize the data used in this report vary by commodity.

Tons, short $(0.9072)=$ tons, metric $(\mathrm{Mt})$

Pounds $/ 2000=$ tons, short

Tons, long $(1.0160469)=$ tons, metric

Troy oz $(0.0311035)=$ kilograms $(\mathrm{kg})$

Kilograms $/ 1000=$ tons, metric

Flask $(.0347)=$ tons, metric 